\title{
Precise Orbit Determination
}

\author{
Adrian Jäggi
}

\section{AIVB}

\section{Astronomical Institute}

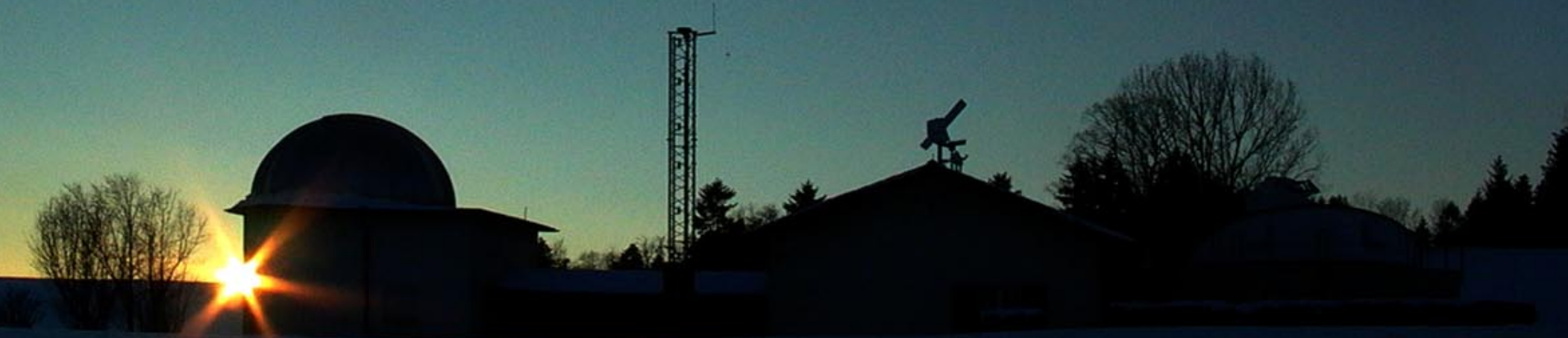




\section{Baknspur des sowj: Enalfnabanten}

Sernbild: Unsa Majon

aufnamme: schulsternwante podewischllighl. , 13. OKt. 1957 . 457 hMEZ

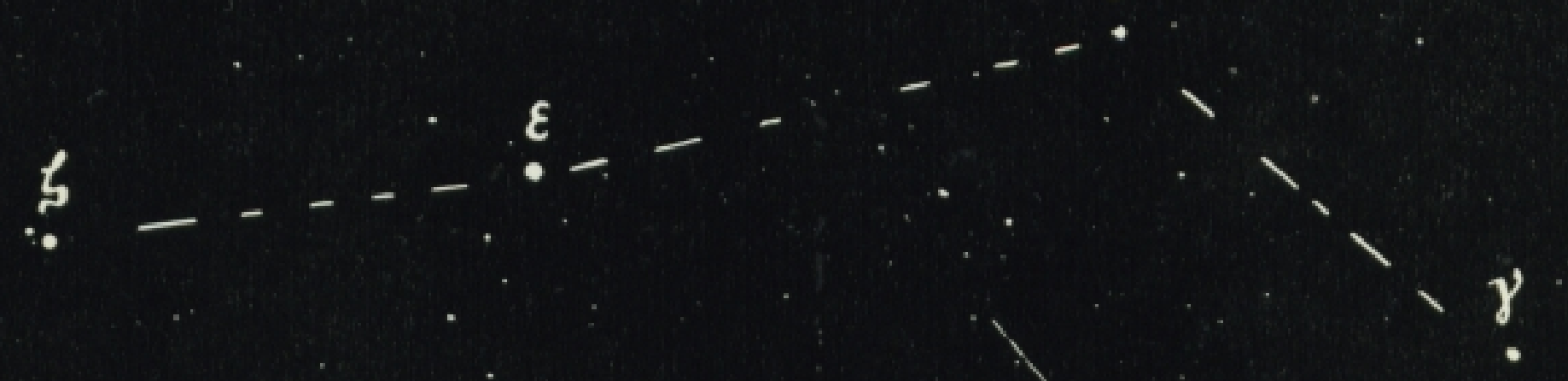

$\eta$ 


\section{Low Earth Orbiters (LEOs)}

\section{CHAMP}

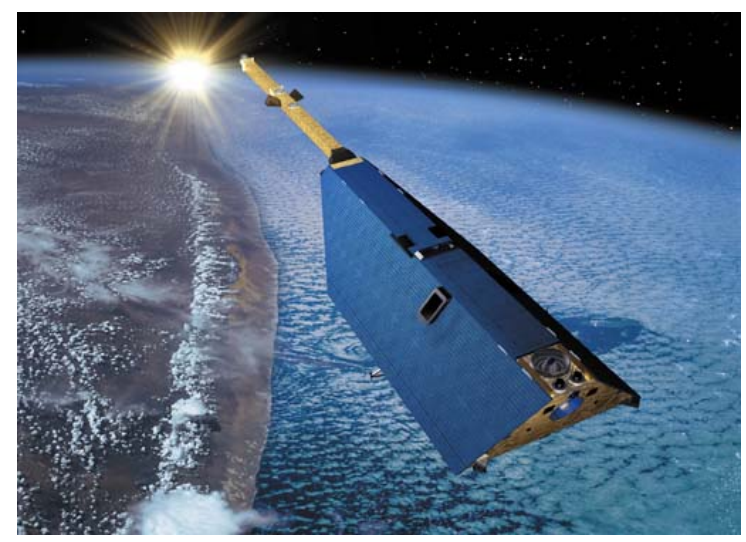

CHAllenging Minisatellite Payload
GRACE

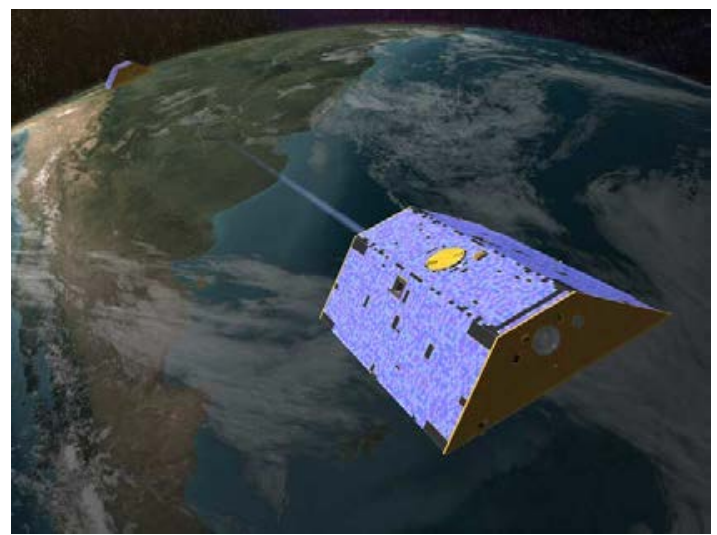

Gravity Recovery And Climate Experiment
GOCE

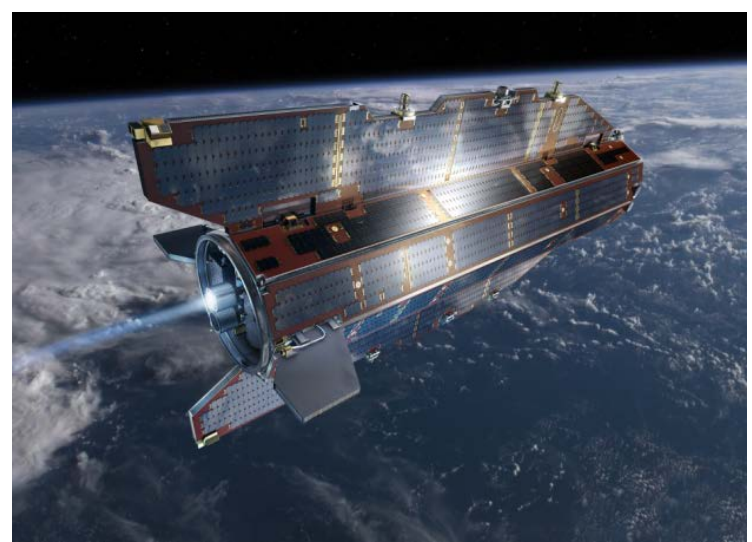

Gravity and steady-state Ocean Circulation Explorer

Of course, there are many more missions equipped with GPS receivers

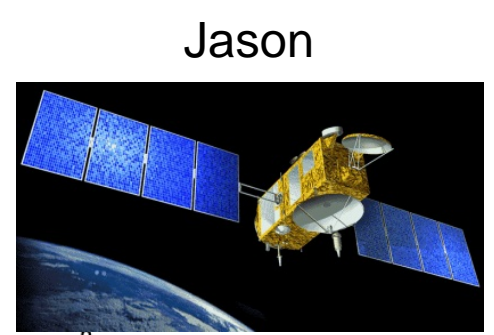
$\boldsymbol{u}^{\circ}$
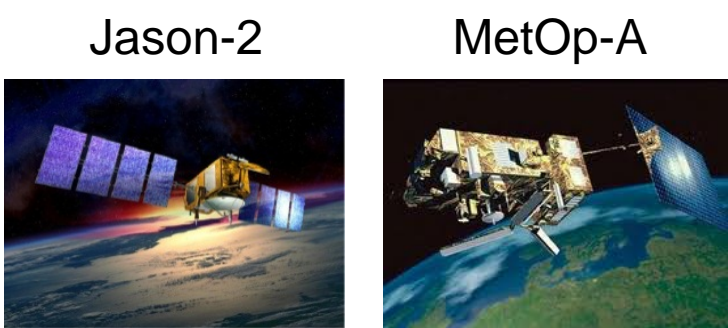

Bad Honnef, 04.10. - 09.10. 2015

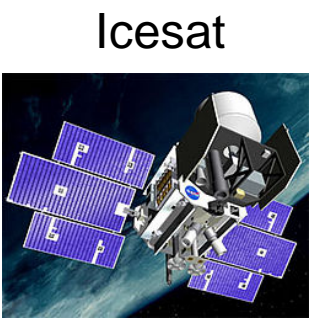

COSMIC

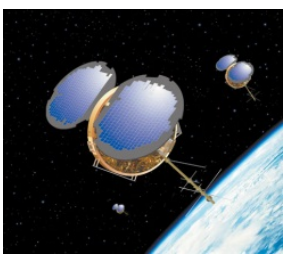

fy

4

Wilhelm und Else Heraeus Autumn School Global Gravity Field Modeling
ellite-to-Satellite Tracking Data 


\section{LEO Constellations}

TanDEM-X

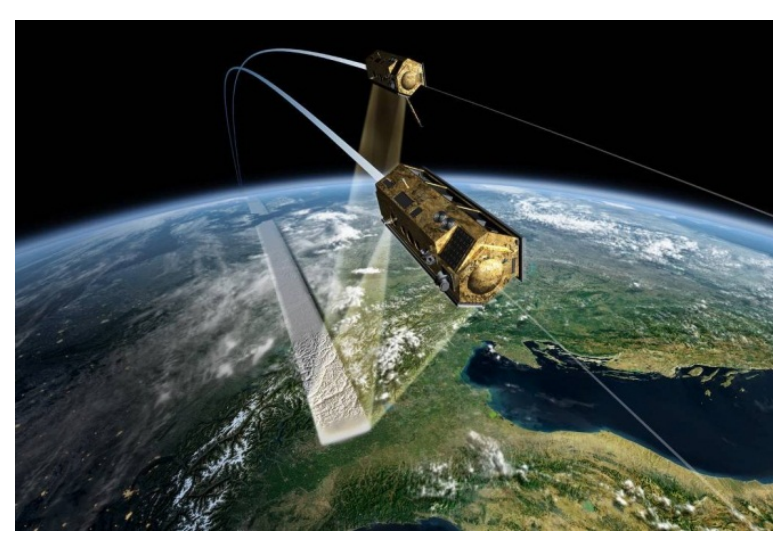

Swarm

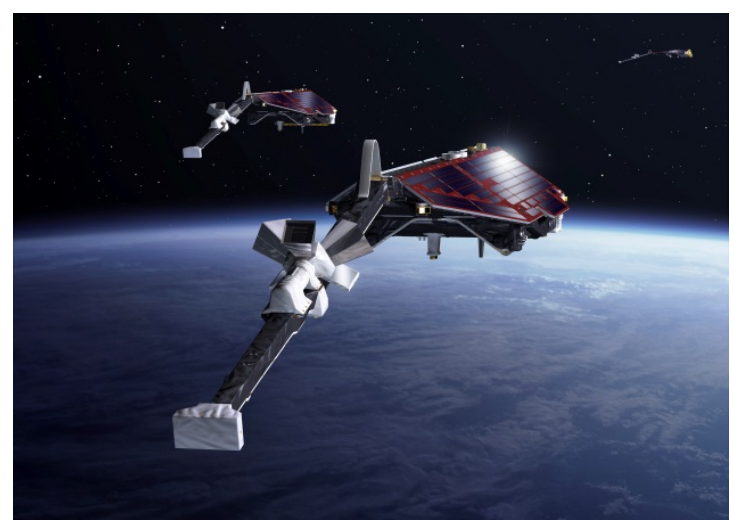

Sentinel

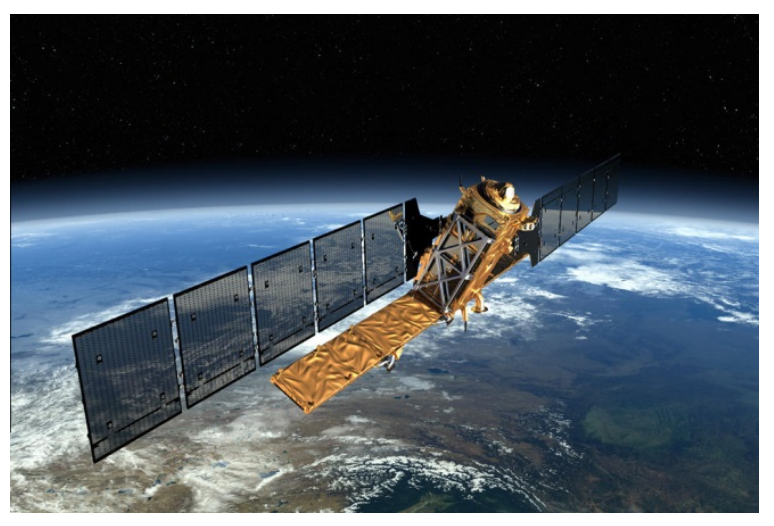

and of course, in the future

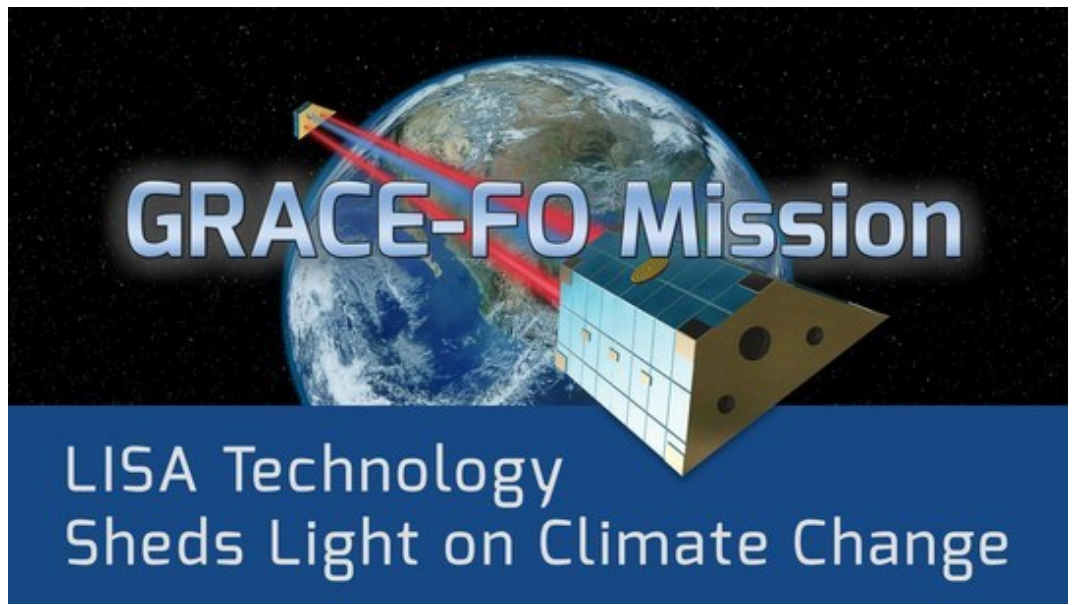

$A / U \boldsymbol{B} u^{u^{s}}$ 


\section{Spherical SLR Satellites}

Starlette

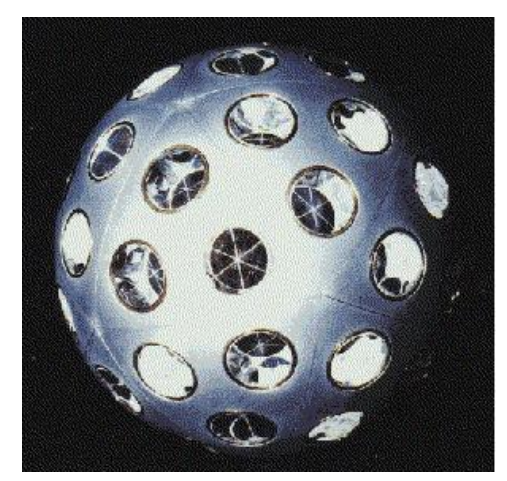

Ajisai

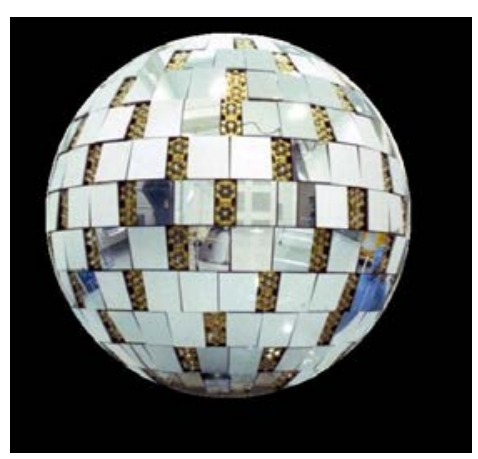

Stella

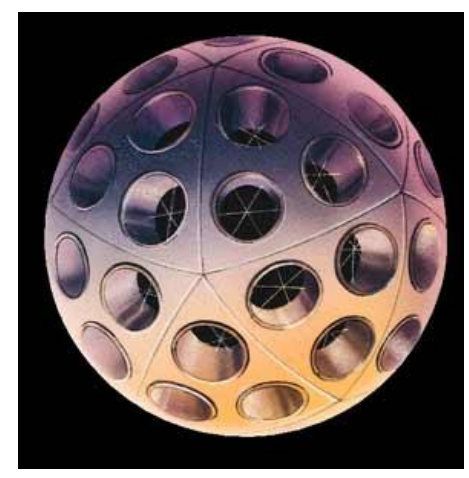

LAGEOS

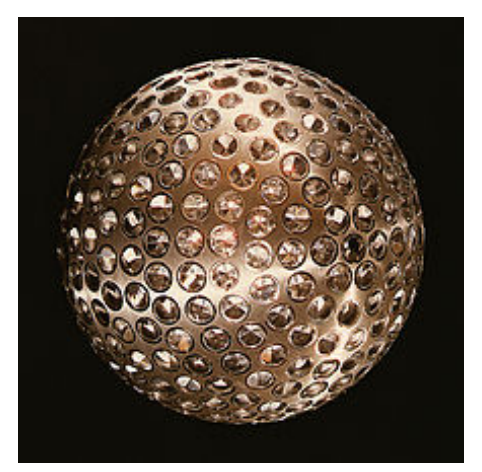

LARES

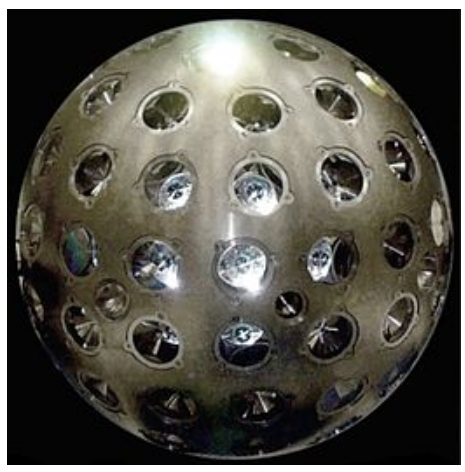

\section{Etalon}

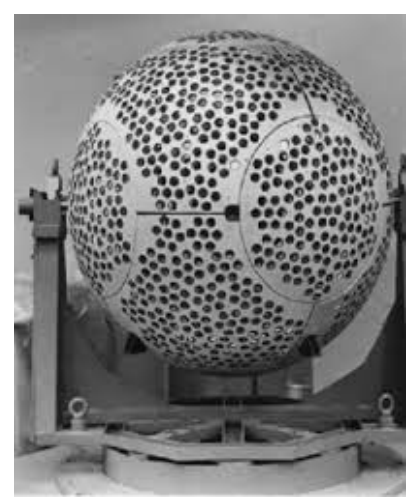




\section{Satellite Laser Ranging}




\section{Introduction to SLR}

SLR: Satellite Laser Ranging

Characteristics:

- Satellites equipped with retro-reflectors

- Global whenever satellites are visible

- Small number of scientific users

- Weather-dependent (optical signals are passing through the atmosphere)

- 1-dimensional distance information 


\section{SLR Space Segment}

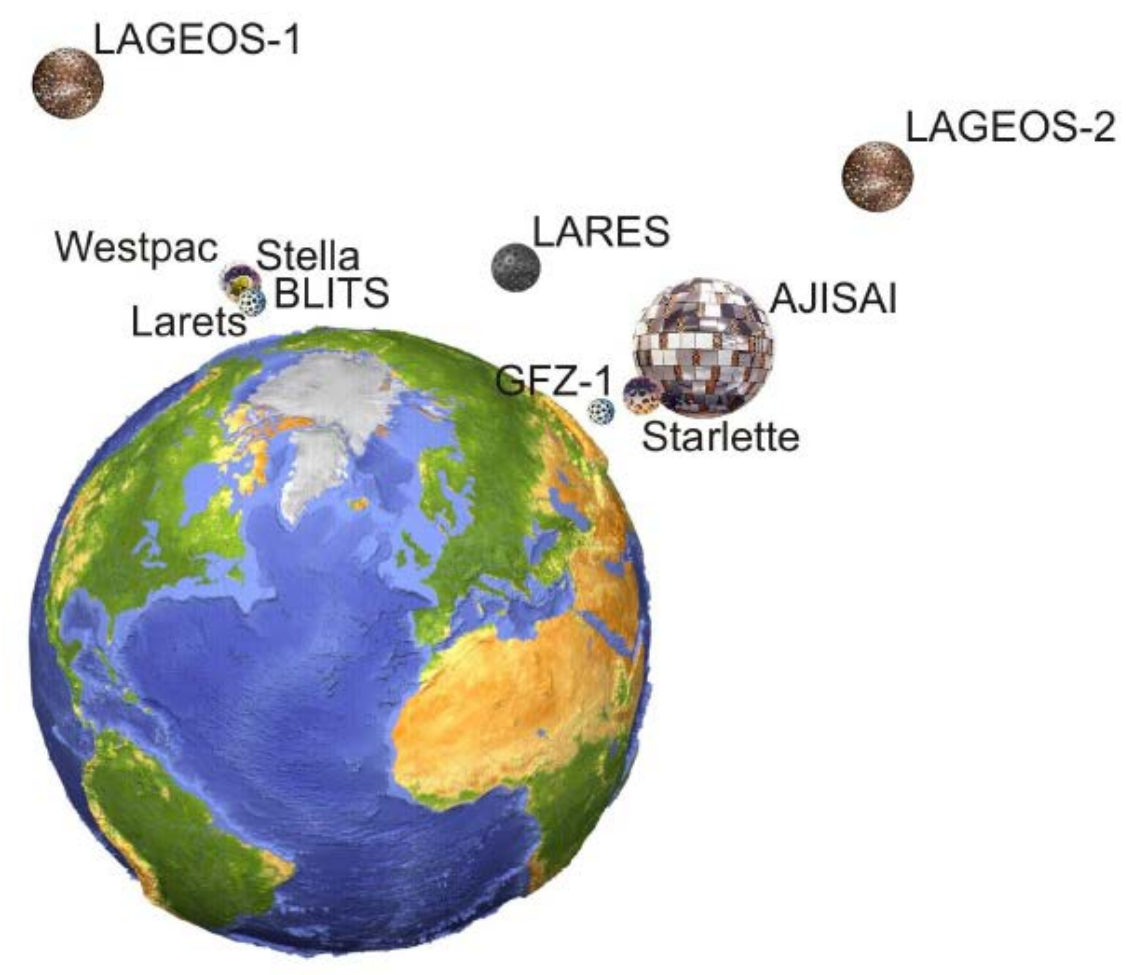

- The space segment is rather small, but long lasting (passive satellites)

- Apart from GFZ-1, Westpac and BLITS, all satellites are still actively used for SLR activities 


\section{SLR Ground Segment}

Each SLR station consists of:

- Laser Oscillator: Neodym-YAG (532 nm) or Titanium-Saphir (423 nm) lasers to generate ultra-short, high-energetic laser pulses

- Optical Telescope: targeted emission of the laser pulses

- Reception System: Optical telescope and detectors to register the incoming photons

- Timing Facility: Epoch registration and time of flight measurement

Most of the SLR stations within the ILRS are unique prototypes 


\section{The Zimmerwald SLR Station}

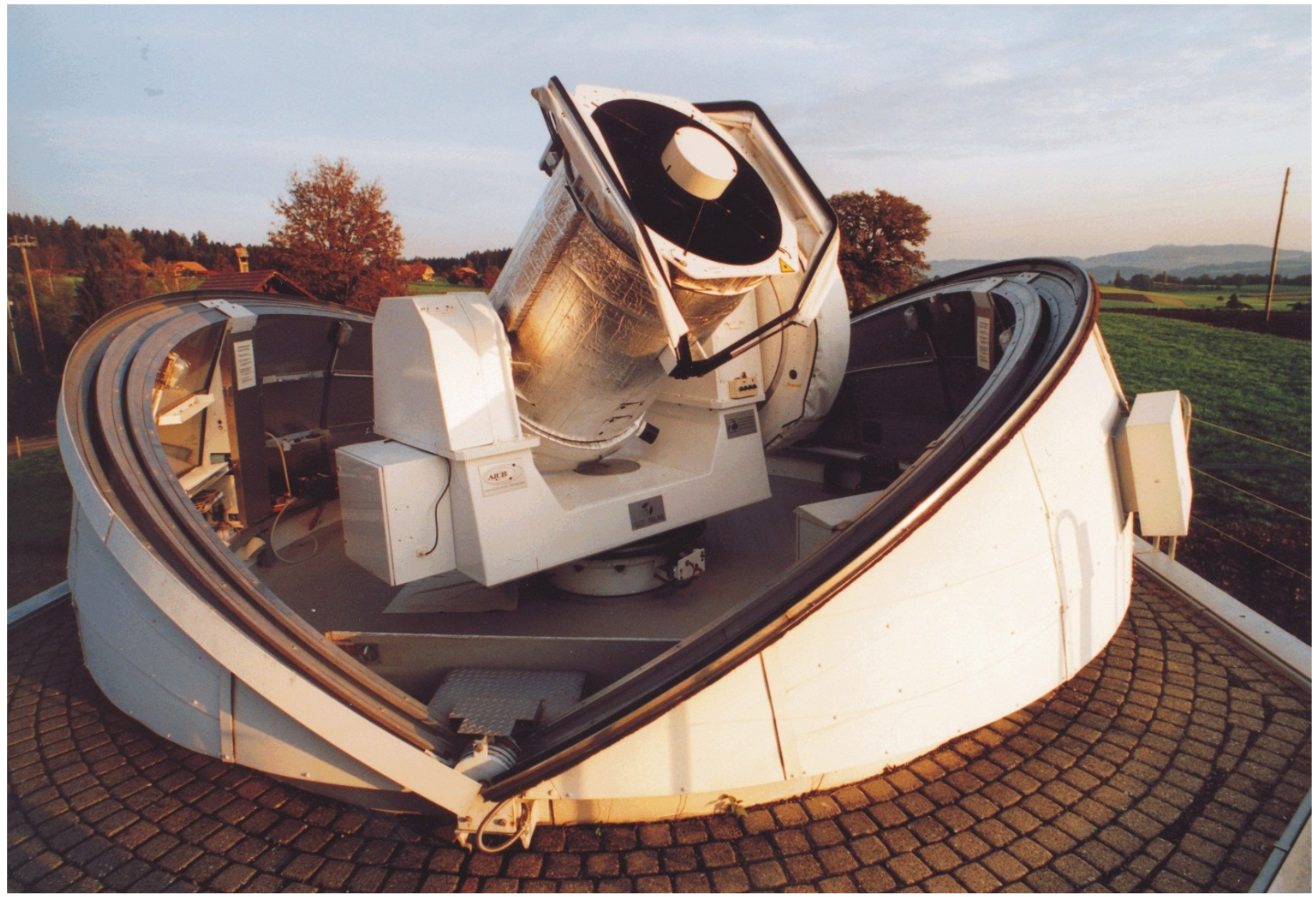




\section{The Zimmerwald SLR Station}

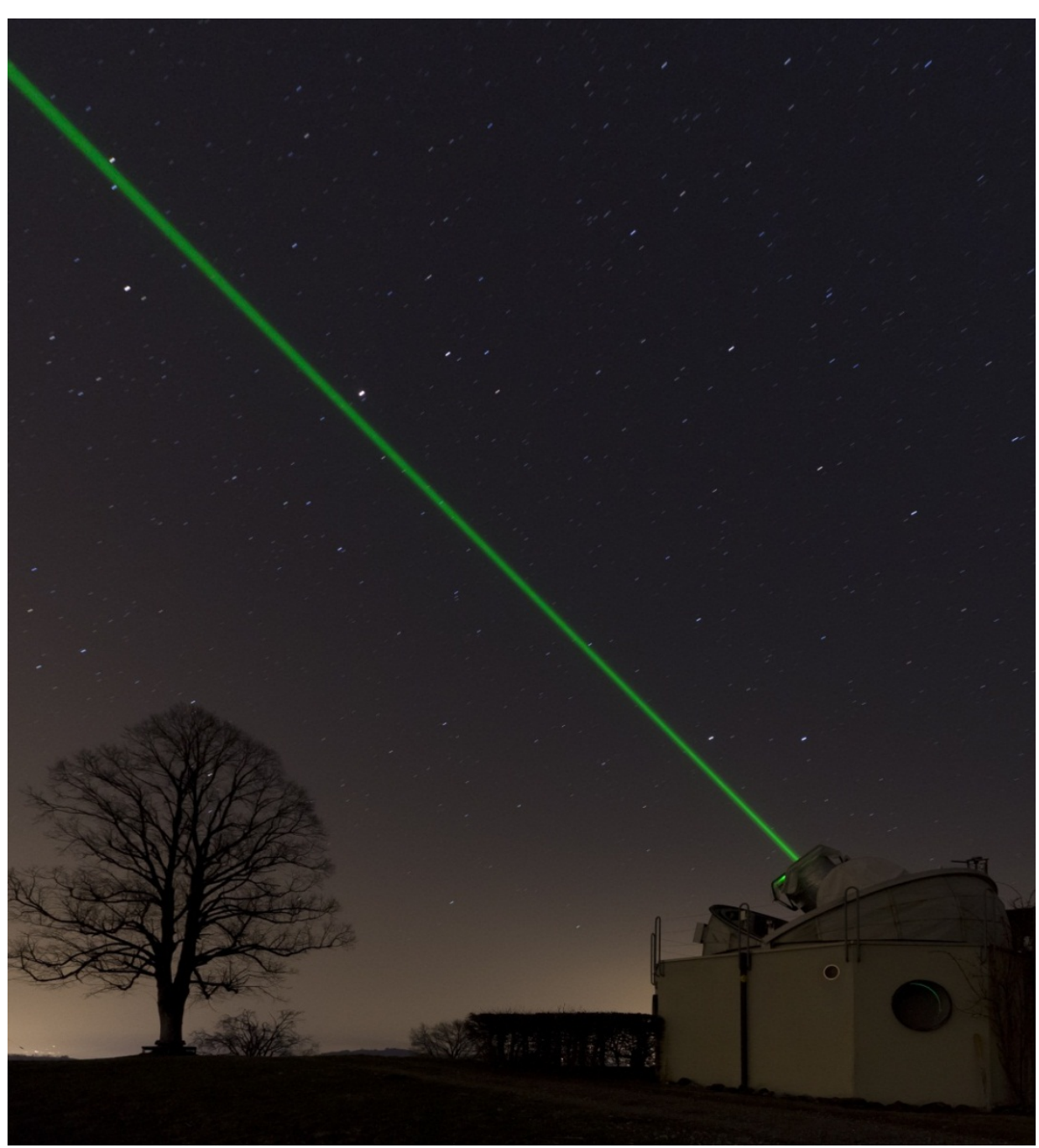




\section{ILRS Station Performance (1)}

ILRS July 1, 2013 through June 30, 2014: Observed Normal Points

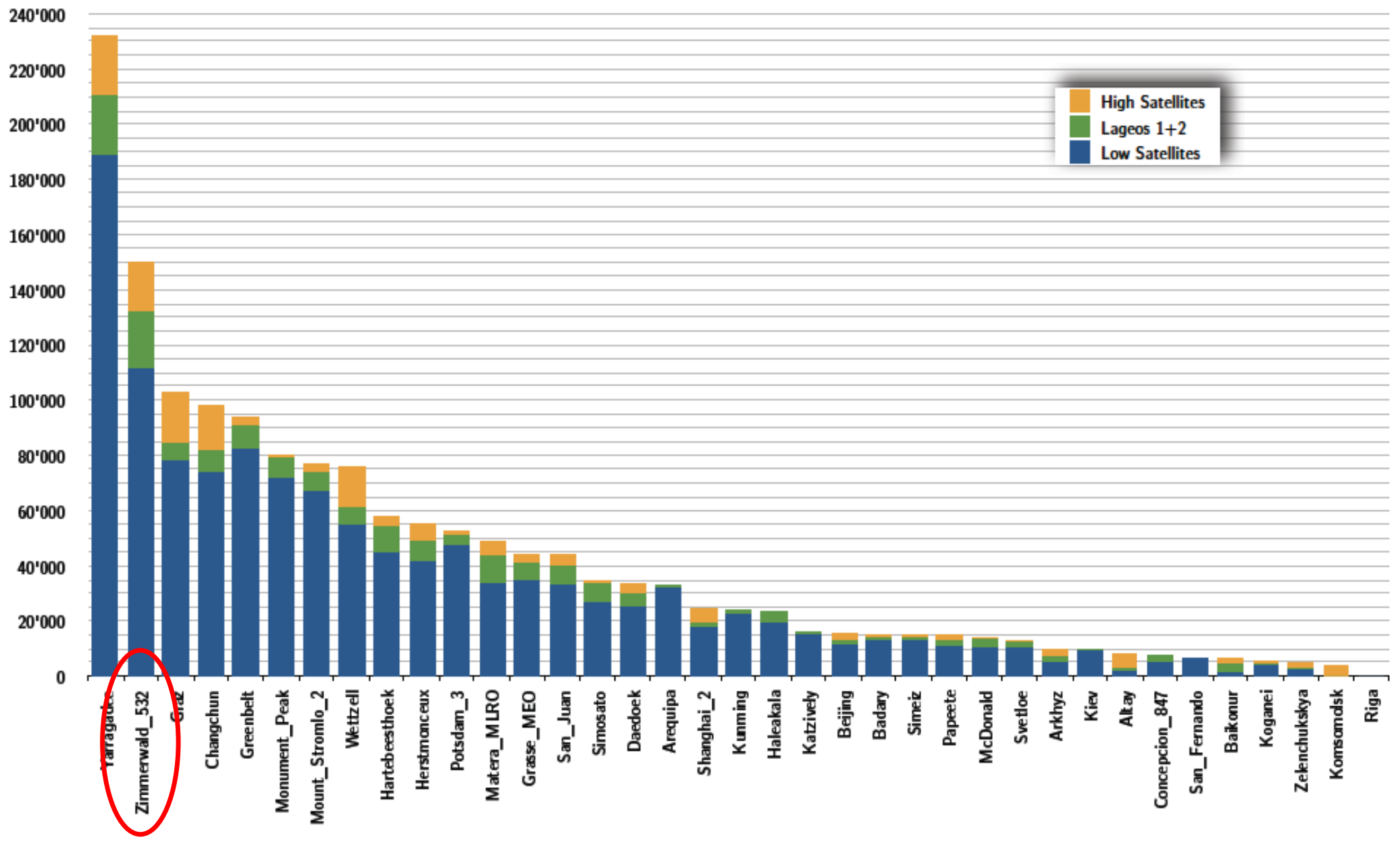




\section{SLR Measurements}

SLR Observations $\Delta t_{i}^{k}$ are defined as:

$$
\Delta t_{i}^{k}=\tau_{i, u p}^{k}+\tau_{i, \text { down }}^{k}
$$

$\Delta t_{i}^{k} \quad$ Round-trip time of flight

$\tau_{i, u p}^{k} \quad$ Time of flight from the laser station to the satellite

$\tau_{i, \text { down }}^{k}$ Time of flight from the satellite back to the laser station

- SLR observations are unbiased „range“ (distance) measurements

- Measurement noise: $\mathrm{mm}$ to $\mathrm{cm}$ for single-shot measurements 


\section{Relation to Position Vectors}

Neglecting atmospheric delays, $\Delta t_{i}^{k}$ may be expressed by:

$$
\Delta t_{i}^{k}=\frac{1}{c}\left(\left|\boldsymbol{r}_{i}\left(t_{\text {sat }}-\tau_{i, u p}^{k}\right)-\boldsymbol{r}^{k}\left(t_{\text {sat }}\right)\right|+\left|\boldsymbol{r}_{i}\left(t_{\text {sat }}+\tau_{i, \text { down }}^{k}\right)-\boldsymbol{r}^{k}\left(t_{\text {sat }}\right)\right|\right)
$$

$\boldsymbol{r}_{i} \quad$ Inertial position of the SLR station at pulse emission and reception time

$r^{k} \quad$ Inertial position of the satellite at pulse reflection time

For terrestrial SLR, $\Delta t_{i}^{k}$ may be approximated by:

$$
\Delta t_{i}^{k}=\frac{2}{c}\left|\boldsymbol{r}_{i}\left(t_{\text {sat }}\right)-\boldsymbol{r}^{k}\left(t_{\text {sat }}\right)\right|
$$




\section{Global Positioning System}

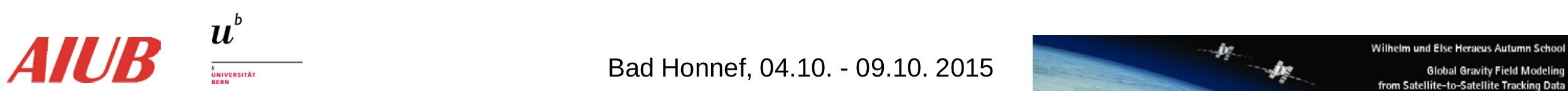




\section{Introduction to GPS}
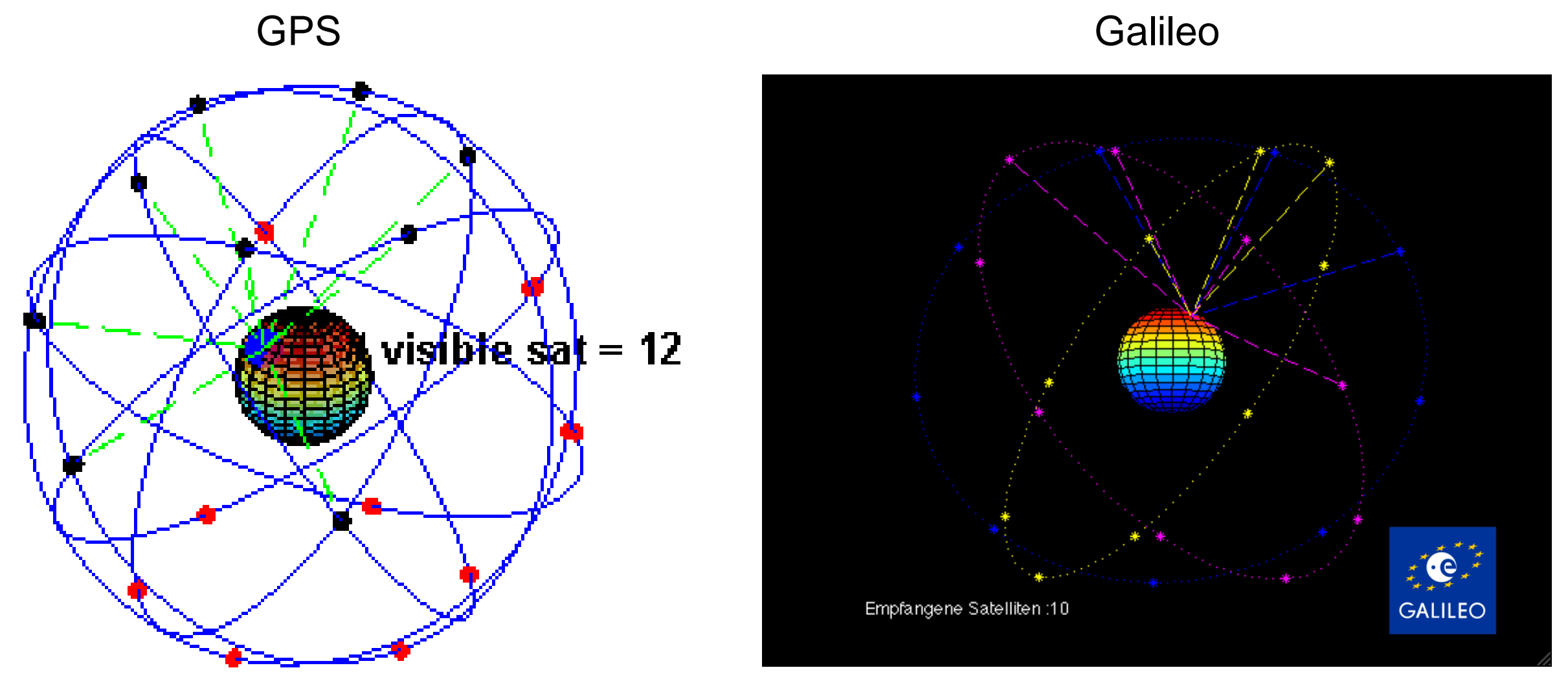

Other GNSS are already existing (GLONASS) or being built up (Galileo), but so far there are no multi-GNSS spaceborne receivers in orbit. 


\section{Introduction to GPS}

GPS: Global Positioning System

Characteristics:

- Satellite system for (real-time) Positioning and Navigation

- Global (everywhere on Earth, up to altitudes of $5000 \mathrm{~km}$ ) and at any time

- Unlimited number of users

- Weather-independent (radio signals are passing through the atmosphere)

- 3-dimensional position, velocity and time information 


\section{GPS Segments}

The GPS consists of 3 main segments:

- Space Segment: the satellites and the constellation of satellites

- Control Segment: the ground stations, infrastructure and software for operation and monitoring of the GPS

- User Segment: all GPS receivers worldwide and the corresponding processing software

We should add an important 4th segment:

- Ground Segment: all civilian permanent networks of reference sites and the international/regional/local services delivering products for the users 


\section{Space Segment}

- The space segment nominally consists of $\mathbf{2 4}$ satellites, presently: 32 active GPS satellites

- Constellation design: at least 4 satellites in view from any location on the Earth at any time

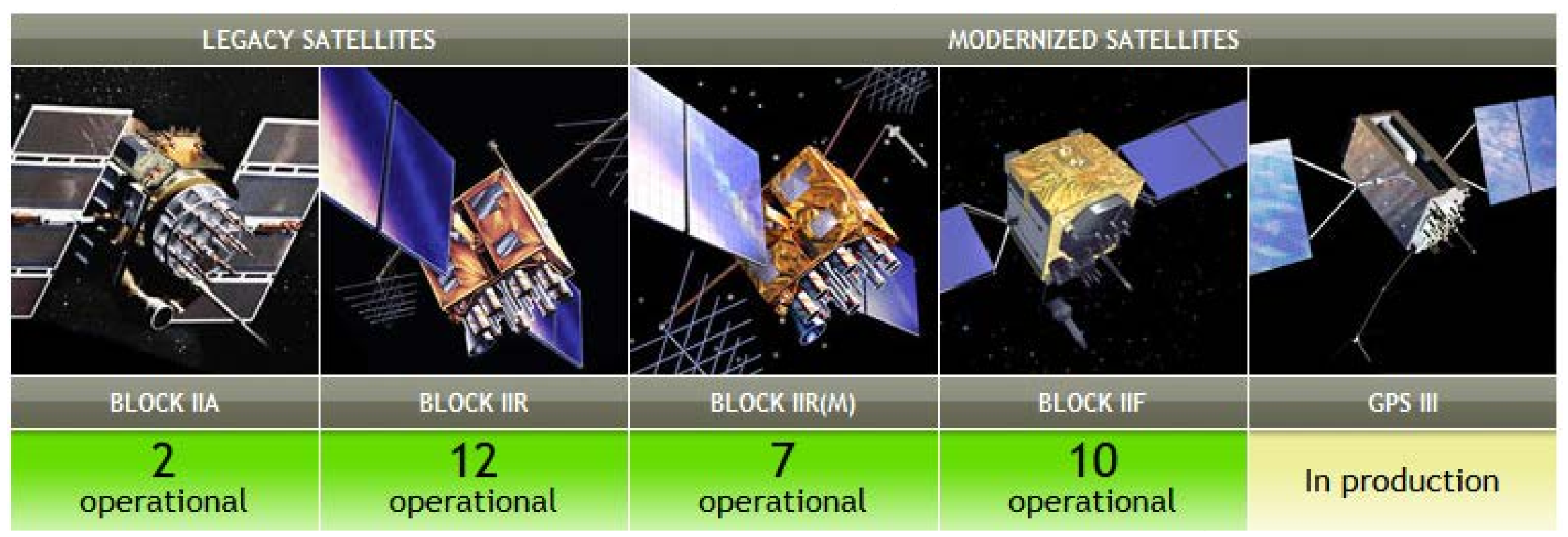
$A / U \boldsymbol{B} \stackrel{u^{b}}{=}$ 


\section{Global Network of the IGS}

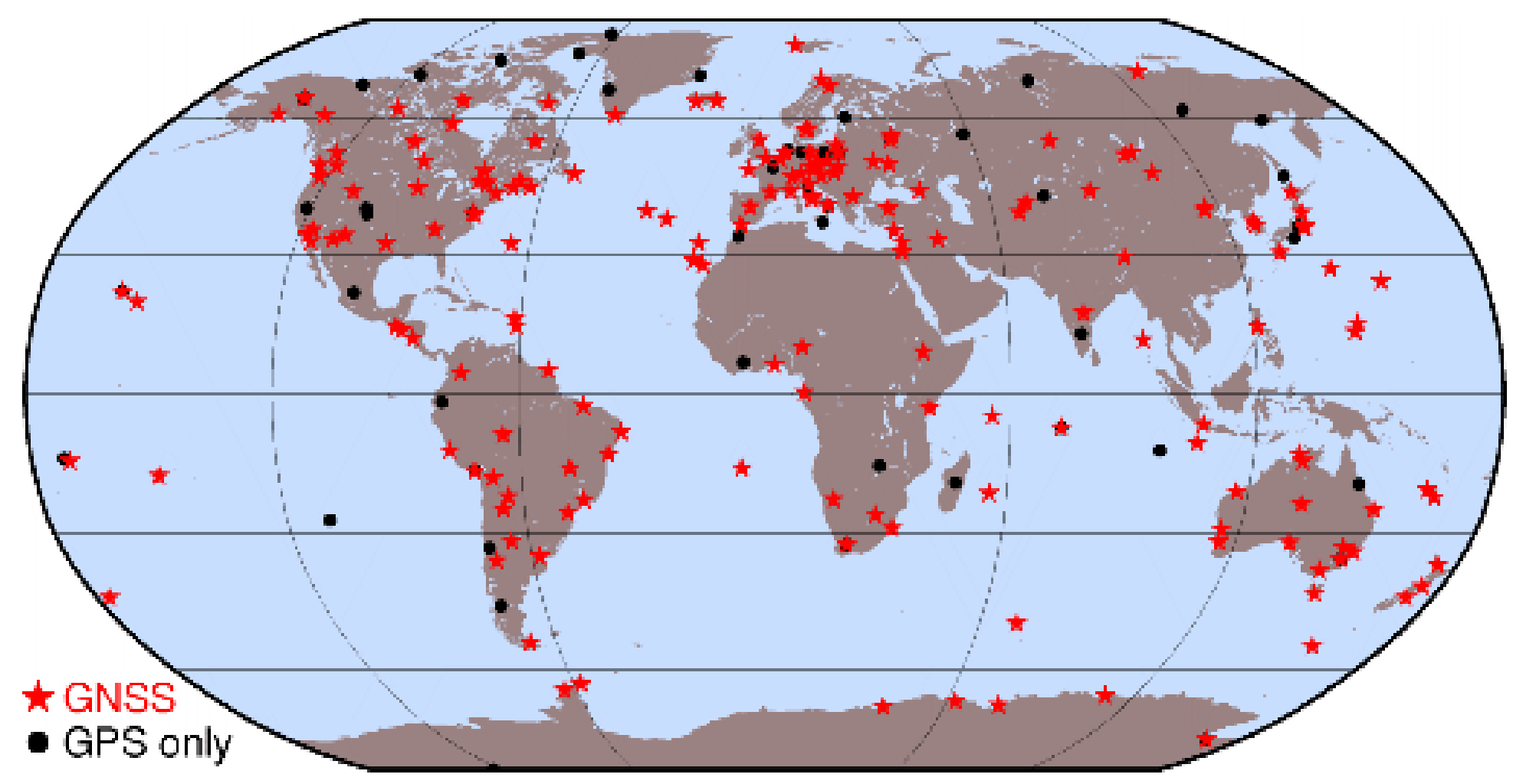

IGS stations used for computation of final orbits at CODE (Dach et al., 2009) 


\section{Parameters of a Global IGS Solution}

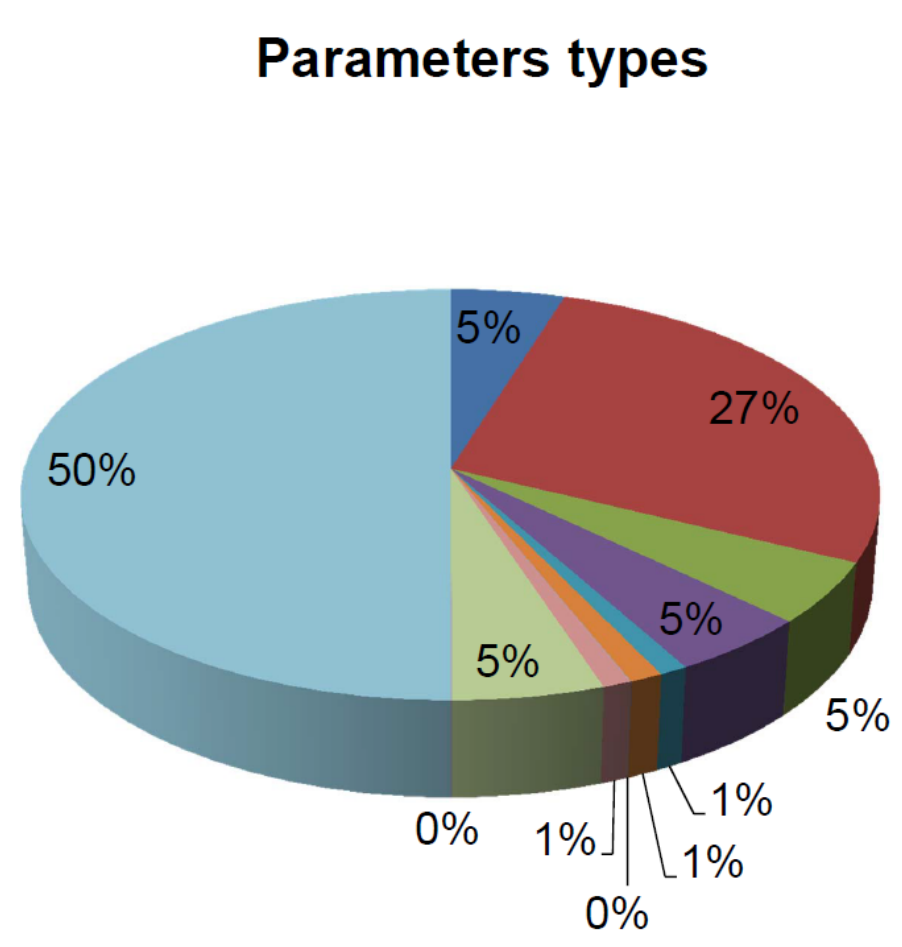

u Station coordinates

- Site-specific troposphere parameters

- Scaling factor for APL model

- Orbital elements

- Stochastic orbit parameters

Earth rotation parameters

Geocenter coordinates

Satellite antenna offset parameters

Satellite antenna pattern

- Scaling factor for higher-order ionosphere

Ambiguity parameters

- Large number of measurement type specific parameters

- Rather small number of orbital parameters 


\section{Performance of IGS Final Orbits}

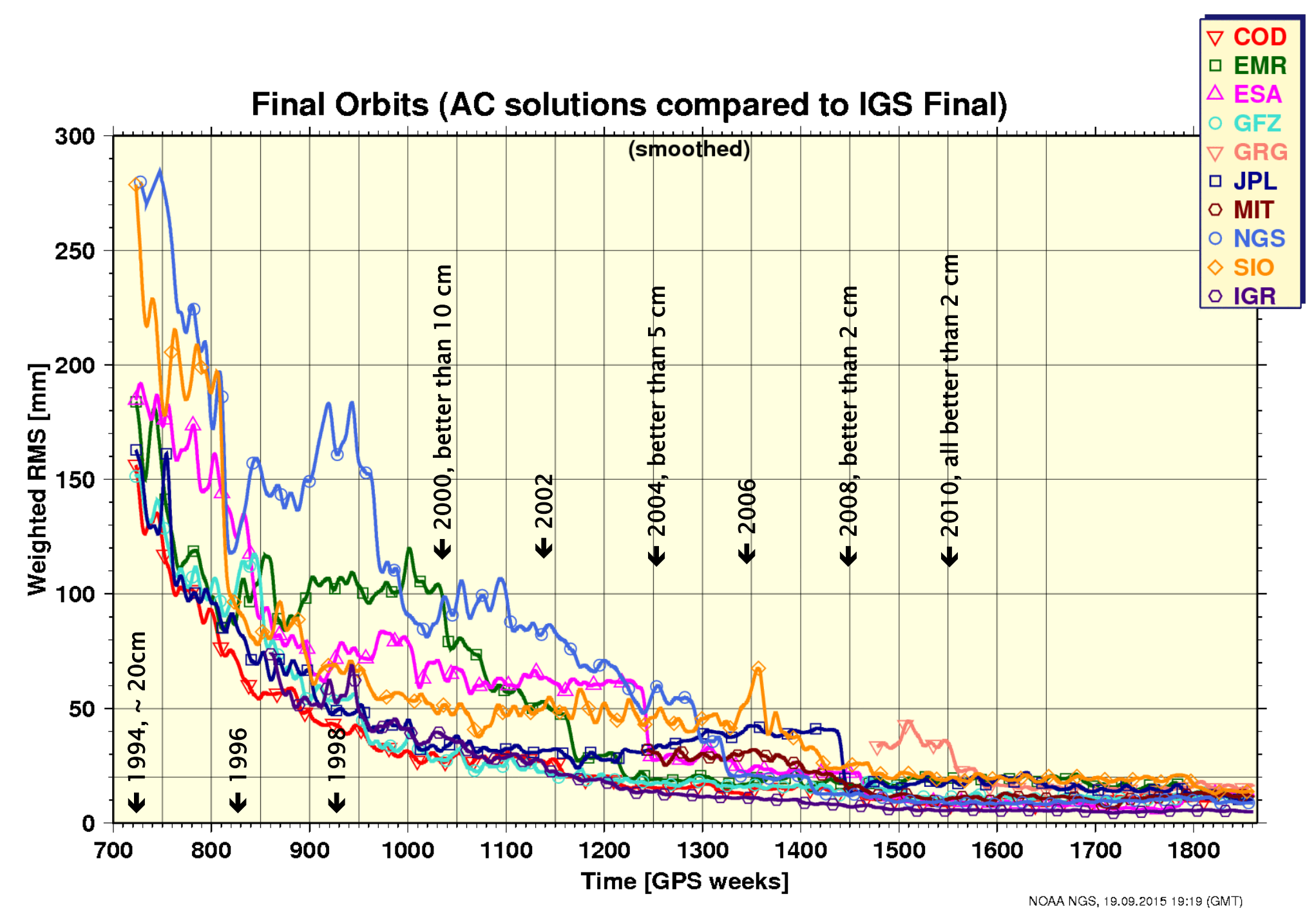




\section{Computation of Final Clocks at CODE}

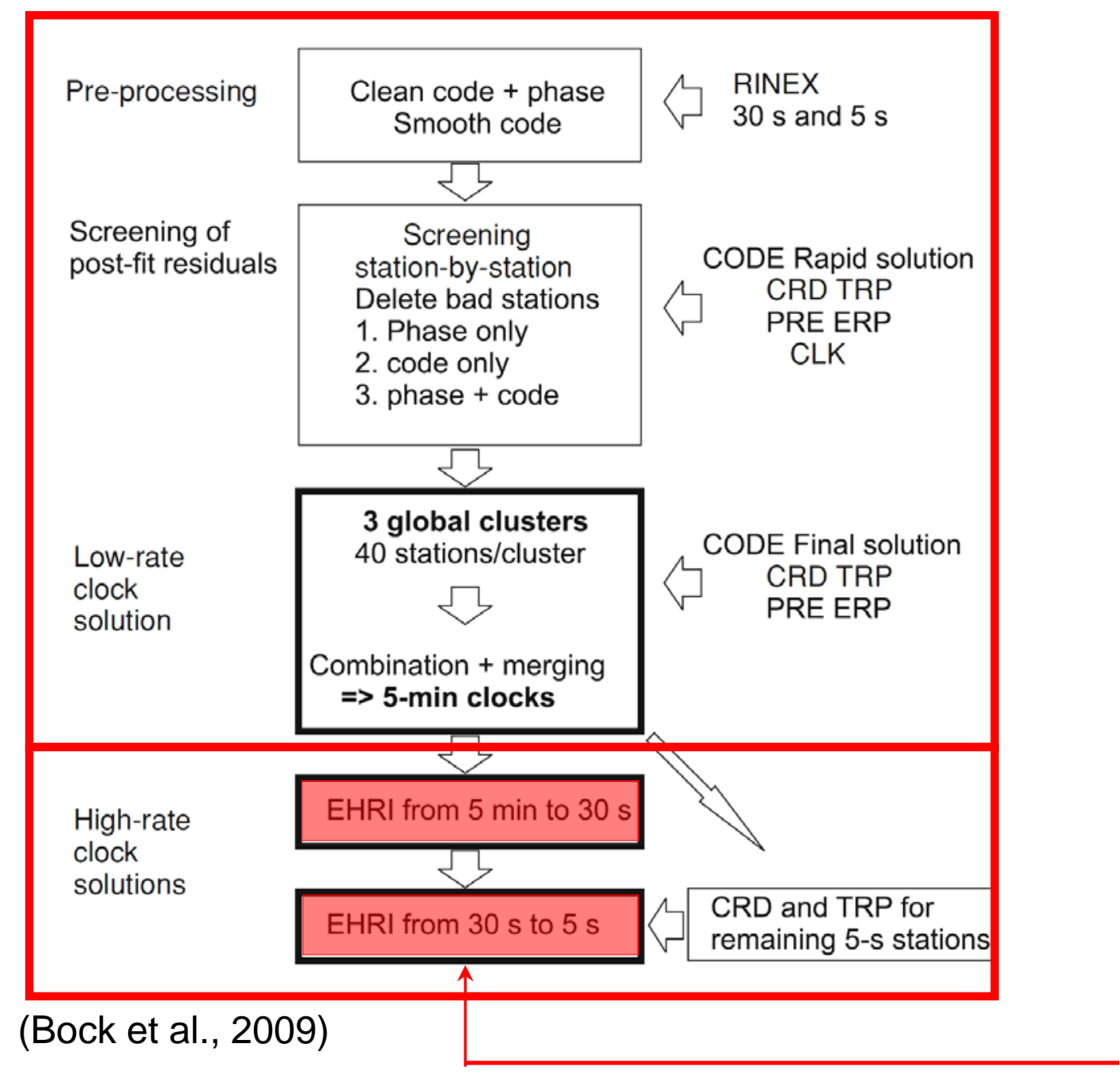

The final clock product with 5 min sampling is based on undifferenced GPS data of typically 120 stations of the IGS network

The IGS $1 \mathrm{~Hz}$ network is finally used for clock densification to $5 \mathrm{sec}$

The $5 \mathrm{sec}$ clocks are interpolated to $1 \mathrm{sec}$ as needed for $1 \mathrm{~Hz}$ LEO GPS data 


\section{GPS Signals}

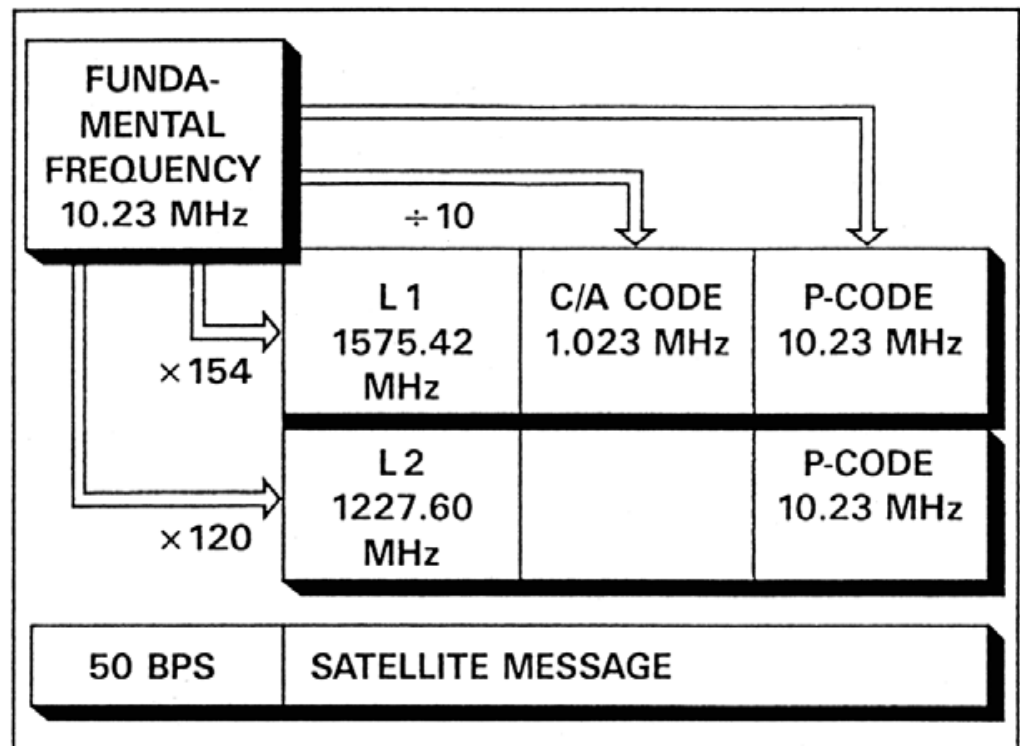

Signals driven by an atomic clock

Two carrier signals (sine waves):

- L1: $f=1575.43 \mathrm{MHz}, \lambda=19 \mathrm{~cm}$

- L2: $f=1227.60 \mathrm{MHz}, \lambda=24 \mathrm{~cm}$

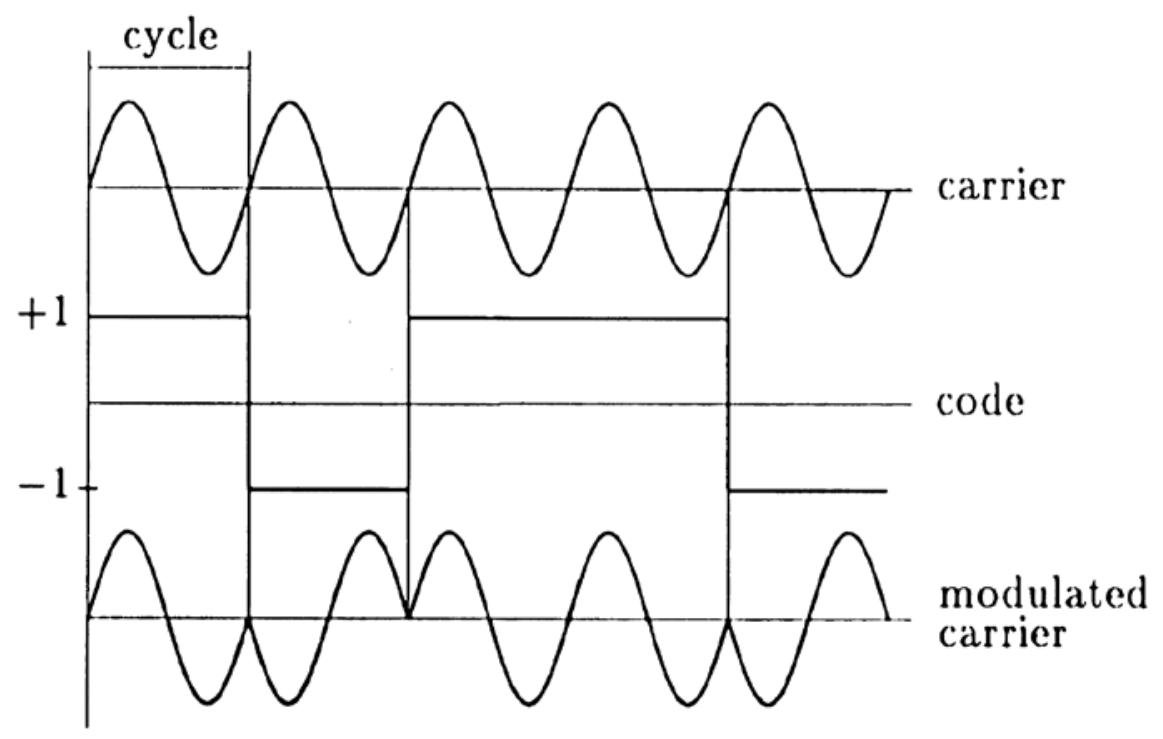

Bits encoded on carrier by phase modulation:

- ClA-code (Clear Access / Coarse Acquisition)

- P-code (Protected / Precise)

- Broadcast/Navigation Message 


\section{Pseudorange / Code Measurements}

Code Observations $P_{i}^{k}$ are defined as:

$$
P_{i}^{k} \doteq c\left(T_{i}-T^{k}\right)
$$

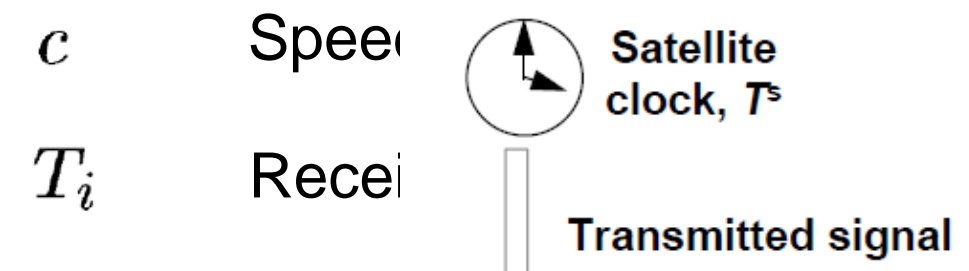

$T^{k} \quad$ GPS

Transmitted signal

Received signal, driven by satellite clock $T^{\text {s }}$

"range" (distance) because of clock offsets

- No actual "range" (distance) because of clock offsets

- Measurementekerpise: $\sim 0.5 \mathrm{~m}$ for P-code (Blewitt, 1997) 


\section{Code Observation Equation}

$$
P_{i}^{k}=\rho_{i}^{k}-c \cdot \Delta t^{k}+c \cdot \Delta t_{i}
$$

$t_{i, t^{k}} \quad$ GPS time of reception and emission

$\Delta t^{k} \quad$ Satellite clock offset $T^{k}-t^{k}$

$\Delta t_{i} \quad$ Receiver clock offset $T_{i}-t_{i}$

$\rho_{i}^{k} \quad$ Distance between receiver and satellite $c\left(t_{i}-t^{k}\right)$

Known from ACs or IGS:

- satellite positions $\left(x^{k_{j}}, y^{k_{j}}, z^{k_{j}}\right)$

- satellite clock offsets $\Delta t^{k_{j}}$
4 unknown parameters:

- receiver position $\left(x_{i}, y_{i}, z_{i}\right)$

- receiver clock offset $\Delta t_{i}$ 


\section{Carrier Phase Measurements (1)}

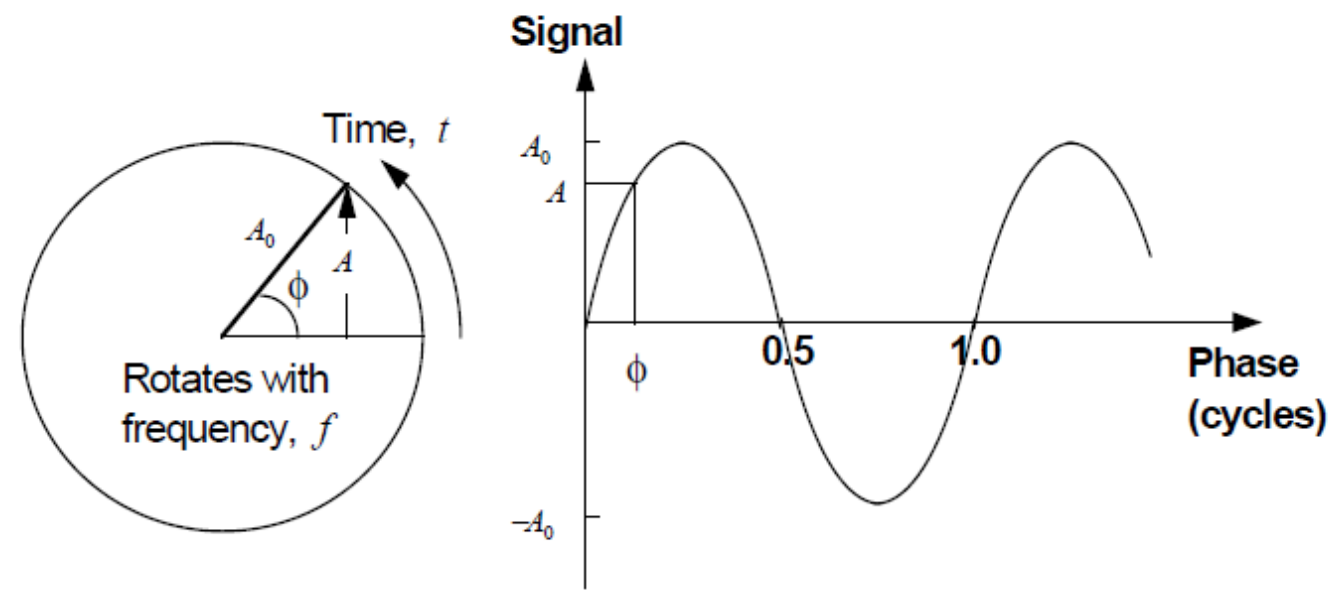

Phase $\phi$ (in cycles) increases linearly with time $t$ :$$
\phi=f \cdot t
$$

where $f$ is the frequency

The satellite generates with its clock the phase signal $\phi^{k}$. At emission time $T^{k}$ (in satellite clock time) we have

$$
\phi^{k}=f \cdot T^{k}
$$

The same phase signal, e.g., a wave crest, propagates from the satellite to the receiver, but the receiver measures only the fractional part of the phase and does not know the integer number of cycles $N_{i}^{k}$ (phase ambiguity):

$$
\phi_{i}^{k}=\phi^{k}-N_{i}^{k}=f \cdot T^{k}-N_{i}^{k}
$$




\section{Carrier Phase Measurements (2)}

The receiver generates with its clock a reference phase. At time of reception $T_{i}$ of the satellite phase $\phi_{i}^{k}$ (in receiver clock time) we have:

$$
\phi_{i}=f \cdot T_{i}
$$

The actual phase measurement is the difference between receiver reference phase $\phi_{i}$ and satellite phase $\phi_{i}^{k}$ :

$$
\psi_{i}^{k}=\phi_{i}-\phi_{i}^{k}=f \cdot T_{i}-\left(f \cdot T^{k}-N_{i}^{k}\right)=f \cdot\left(T_{i}-T^{k}\right)+N_{i}^{k}
$$

Multiplication with the wavelength $\lambda=c / f$ leads to the phase observation equation in meters:

$$
\begin{aligned}
L_{i}^{k} & =\lambda \cdot \psi_{i}^{k}=c \cdot\left(T_{i}-T^{k}\right)+\lambda \cdot N_{i}^{k} \\
& =\rho_{i}^{k}-c \cdot \Delta t^{k}+c \cdot \Delta t_{i}+\lambda \cdot N_{i}^{k}
\end{aligned}
$$

Difference to the pseudorange observation: integer ambiguity term $N_{i}^{k}$ 


\section{Improved Observation Equation}

$$
\begin{aligned}
L_{i}^{k}=\rho_{i}^{k} & -c \cdot \Delta t^{k}+c \cdot \Delta t_{i}+\mathbf{X}_{i}^{k}+\lambda \cdot N_{i}^{k} \\
& +\Delta_{r e l}-c \cdot b^{k}+c \cdot b_{i}+m_{i}^{k}+\epsilon_{i}^{k}
\end{aligned}
$$

$\rho_{i}^{k} \quad$ Distance between satellite and receiver $\longleftarrow$ Satellite positions and clocks $\Delta t^{k} \quad$ Satellite clock offset wrt GPS time 4 are known from ACs or IGS $\Delta t_{i} \quad$ Receiver clock offset wrt GPS time

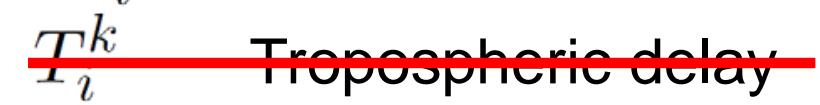

$I_{i}^{k}$ inosprevic delay

$N_{i}^{k} \quad$ Phase ambiguity

$\Delta_{r e l} \quad$ Relativistic corrections

$b^{k} \quad$ Delays in satellite (cables, electronics)

$b_{i} \quad$ Delays in receiver and antenna

$m_{i}^{k} \quad$ Multipath, scattering, bending effects

$\epsilon_{i}^{k} \quad$ Measurement error

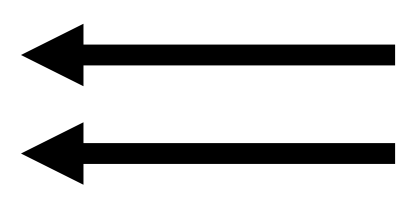

Not existent for LEOs

Cancels out (first order only) when forming the ionospherefree linear combination:

$$
L_{c}=\frac{f_{1}^{2}}{f_{1}^{2}-f_{2}^{2}} L_{1}-\frac{f_{2}^{2}}{f_{1}^{2}-f_{2}^{2}} L_{2}
$$




\section{Geometric Distance}

Geometric distance $\rho_{\text {leo }}^{k}$ is given by:

$$
\rho_{\text {leo }}^{k}=\left|\boldsymbol{r}_{\text {leo }}\left(t_{l e o}\right)-\boldsymbol{r}^{k}\left(t_{l e o}-\tau_{\text {leo }}^{k}\right)\right|
$$

$\boldsymbol{r}_{\text {leo }}$ Inertial position of LEO antenna phase center at reception time $\boldsymbol{r}^{k} \quad$ Inertial position of GPS antenna phase center of satellite $k$ at emission time $\tau_{l e o}^{k} \quad$ Signal traveling time between the two phase center positions

Different ways to represent $\boldsymbol{r}_{l e o}$ :

- Kinematic orbit representation

- Dynamic or reduced-dynamic orbit representation 


\section{Orbit Representation}




\section{Kinematic Orbit Representation (1)}

Satellite position $\boldsymbol{r}_{l e o}\left(t_{l e o}\right)$ (in inertial frame) is given by:

$$
\boldsymbol{r}_{l e o}\left(t_{l e o}\right)=\boldsymbol{R}\left(t_{l e o}\right) \cdot\left(\boldsymbol{r}_{l e o, e, 0}\left(t_{l e o}\right)+\delta \boldsymbol{r}_{l e o, e, a n t}\left(t_{l e o}\right)\right)
$$

$\boldsymbol{R}$

Transformation matrix from Earth-fixed to inertial frame

$\boldsymbol{r}_{l e o, e, 0}$

LEO center of mass position in Earth-fixed frame

$\delta \boldsymbol{r}_{l e o, e, a n t}$

LEO antenna phase center offset in Earth-fixed frame

Kinematic positions $\boldsymbol{r}_{l e o, e, 0}$ are estimated for each measurement epoch:

- Measurement epochs need not to be identical with nominal epochs

- Positions are independent of models describing the LEO dynamics. Velocities cannot be provided 


\section{Kinematic Orbit Representation (2)}

A kinematic orbit is an ephemeris at discrete measurement epochs

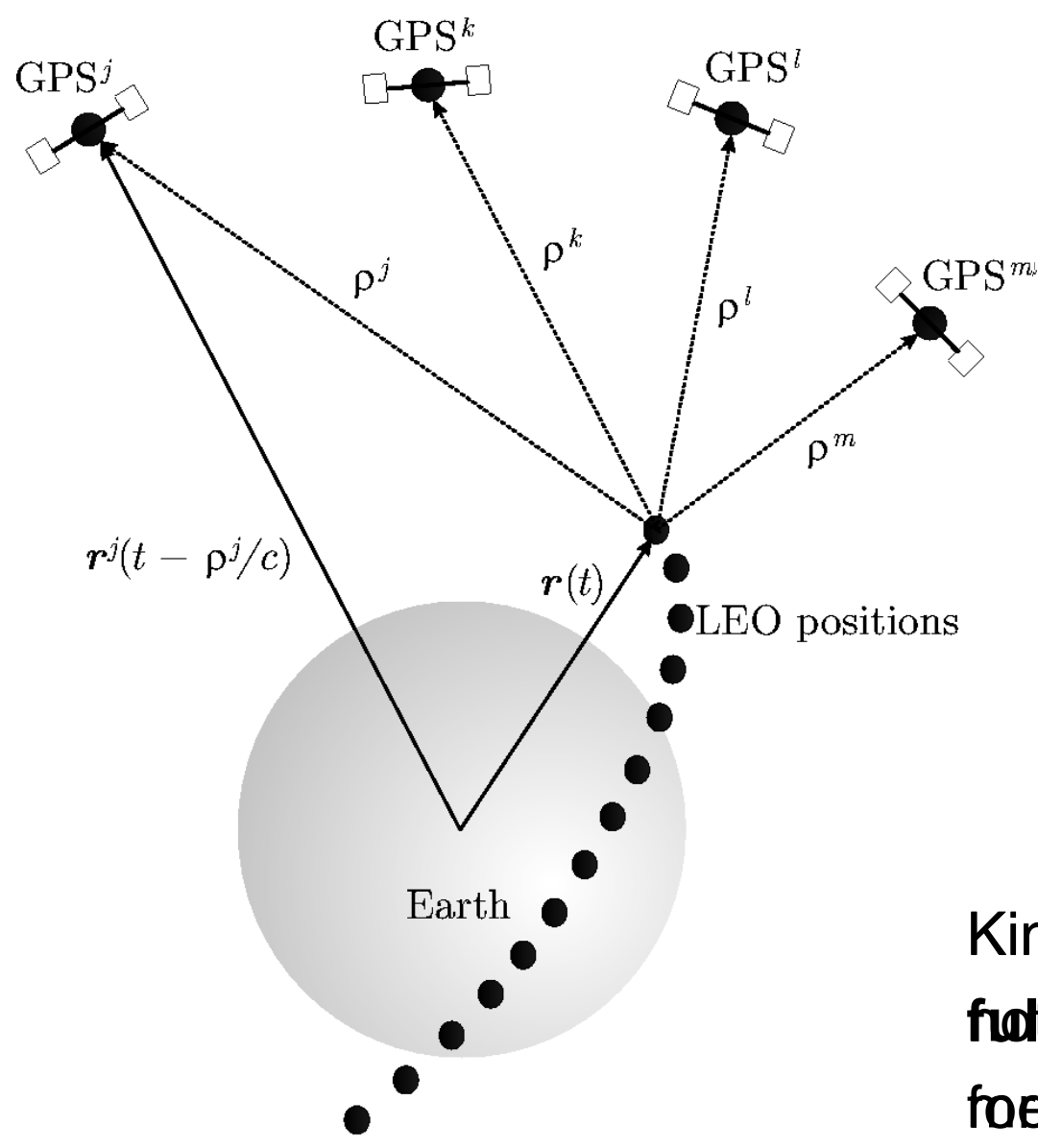

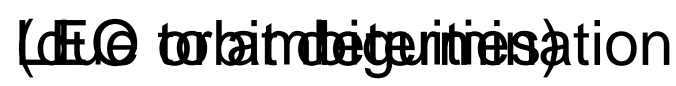

Kinematic positions are

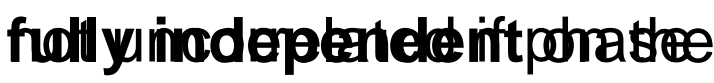
forcesumededstisstel fiosed

(Sägblæaab, Rothla)cher, 2004) 


\section{Kinematic Orbit Representation (3)}

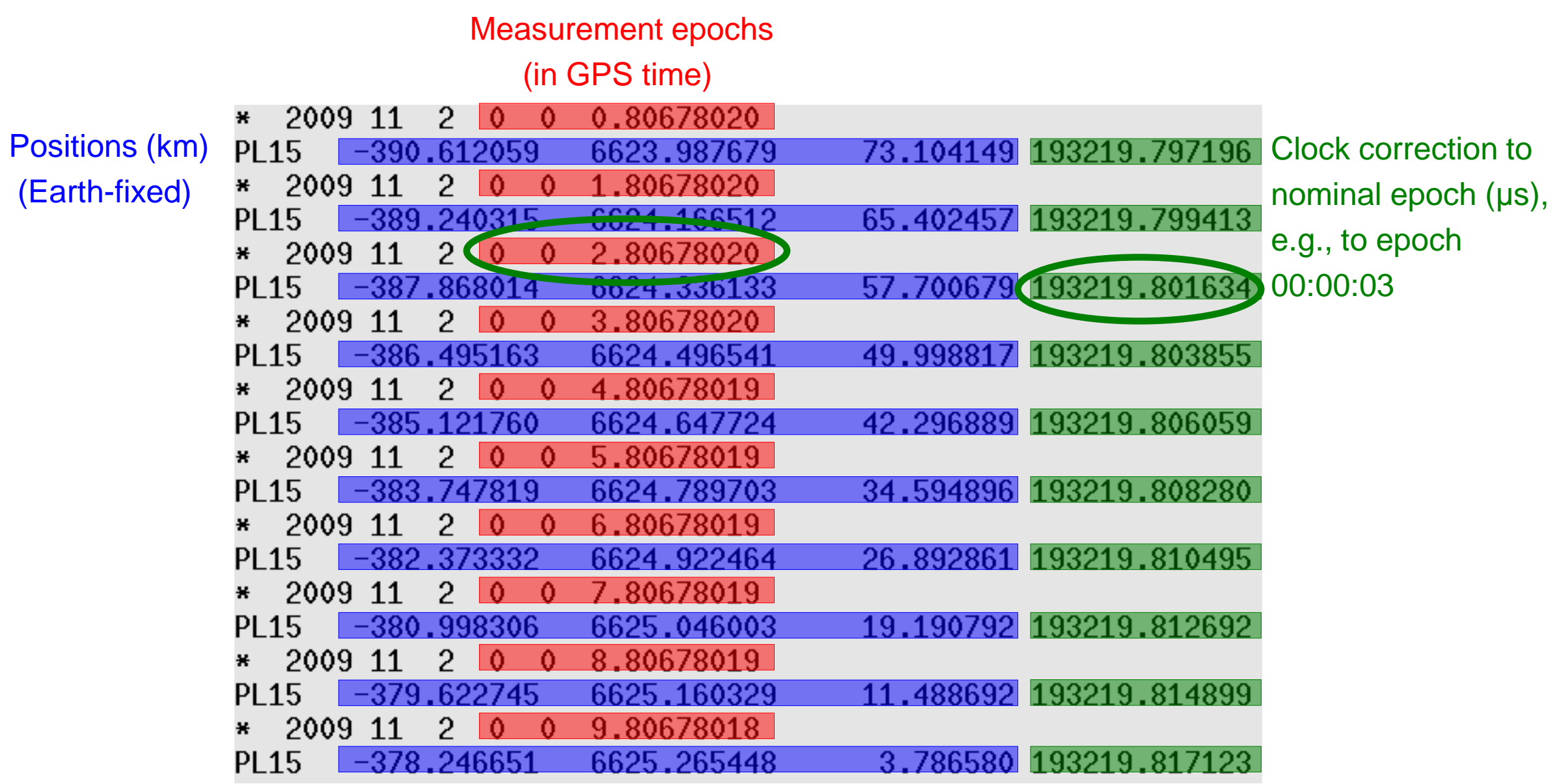

Excerpt of kinematic GOCE positions at begin of 2 Nov, 2009 GO_CONS_SST_PKI_2_20091101T235945_20091102T235944_0001 Times in UTC $\boldsymbol{A} / \boldsymbol{U} \boldsymbol{B} \stackrel{u^{b}}{=}$ Bad Honnef, 04.10. - 09.10. 2015

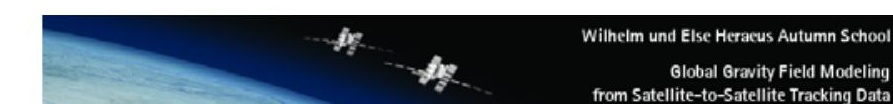




\section{Dynamic Orbit Representation (1)}

Satellite position $\boldsymbol{r}_{l e o}\left(t_{l e o}\right)$ (in inertial frame) is given by:

$$
\boldsymbol{r}_{l e o}\left(t_{l e o}\right)=\boldsymbol{r}_{l e o, 0}\left(t_{l e o} ; a, e, i, \Omega, \omega, u_{0} ; Q_{1}, \ldots, Q_{d}\right)+\delta \boldsymbol{r}_{l e o, a n t}\left(t_{l e o}\right)
$$

$\boldsymbol{r}_{l e o, 0}$

LEO center of mass position

$\delta \boldsymbol{r}_{\text {leo,ant }}$

LEO antenna phase center offset

$a, e, i, \Omega, \omega, u_{0}$

LEO initial osculating orbital elements

$Q_{1}, \ldots, Q_{d}$

LEO dynamical parameters

Satellite trajectory $\boldsymbol{r}_{l e o, 0}$ is a particular solution of an equation of motion

- One set of initial conditions (orbital elements) is estimated per arc.

Dynamical parameters of the force model on request 


\section{Dynamic Orbit Representation (2)}

Equation of motion (in inertial frame) is given by:

$$
\ddot{\boldsymbol{r}}=-G M \frac{\boldsymbol{r}}{r^{3}}+\boldsymbol{f}_{1}\left(t, \boldsymbol{r}, \dot{\boldsymbol{r}}, Q_{1}, \ldots, Q_{d}\right)
$$

with initial conditions

$$
\begin{aligned}
\boldsymbol{r}\left(t_{0}\right) & =\boldsymbol{r}\left(a, e, i, \Omega, \omega, u_{0} ; t_{0}\right) \\
\dot{\boldsymbol{r}}\left(t_{0}\right) & =\dot{\boldsymbol{r}}\left(a, e, i, \Omega, \omega, u_{0} ; t_{0}\right)
\end{aligned}
$$

The acceleration $\boldsymbol{f}_{1}$ consists of gravitational and non-gravitational perturbations taken into account to model the satellite trajectory. Unknown parameters $Q_{1}, \ldots, Q_{d}$ of force models may appear in the equation of motion together with deterministic (known) accelerations given by analytical models. 


\section{Perturbing Accelerations of a LEO Satellite}

\section{Force}

Central term of Earth's gravity field

Oblateness of Earth's gravity field

Atmospheric drag

Higher order terms of Earth's gravity field

Attraction from the Moon

Attraction from the Sun

Direct solar radiation pressure

\section{Acceleration}

$\left(\mathrm{m} / \mathrm{s}^{2}\right)$

8.42

0.015

0.00000079

0.00025

0.0000054

0.0000005

0.000000097 


\section{Osculating Orbital Elements (1)}

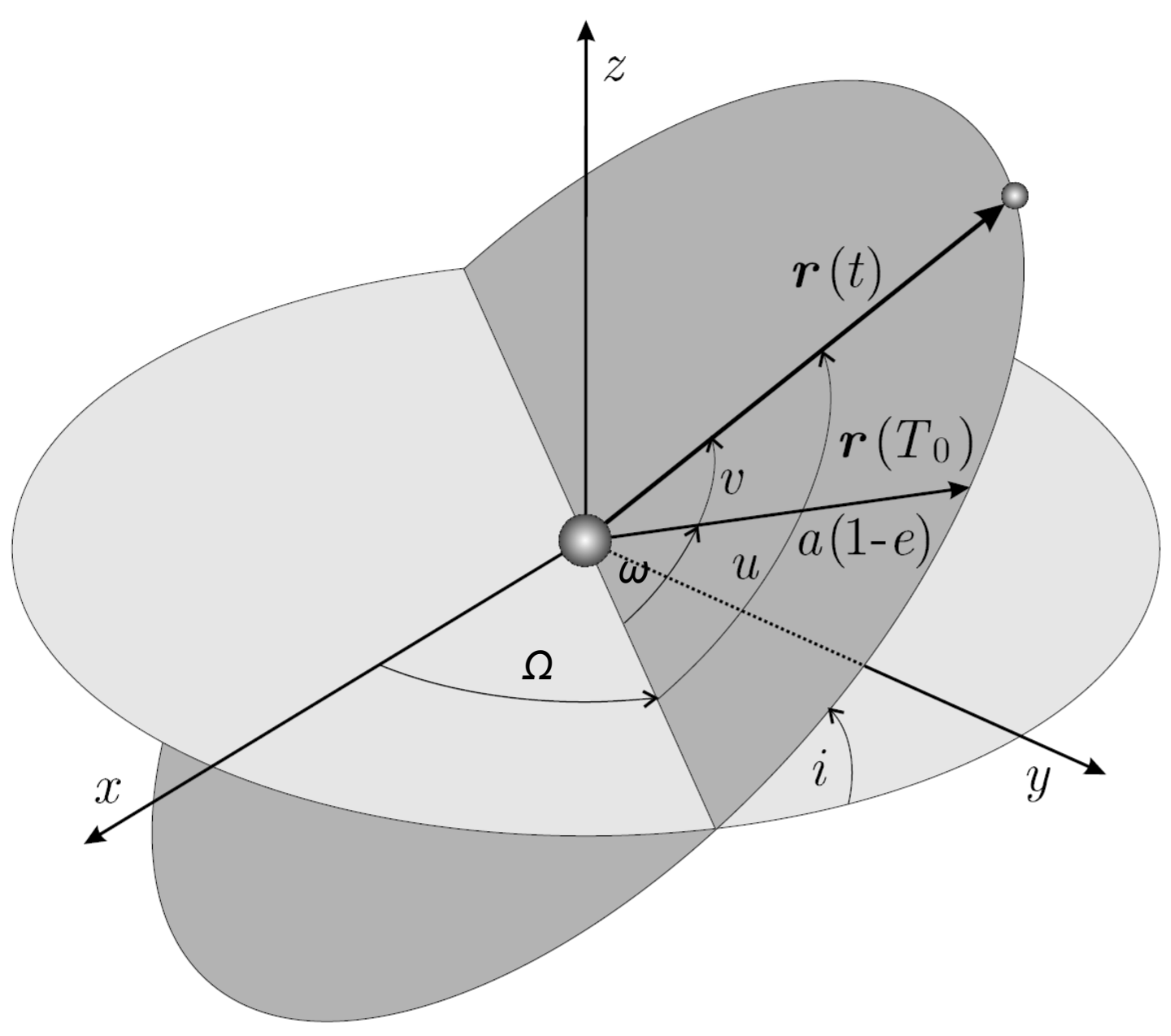

$A / U \boldsymbol{B}$

Bad Honnef, 04.10. - 09.10. 2015 


\section{Osculating Orbital Elements of GOCE (2)}

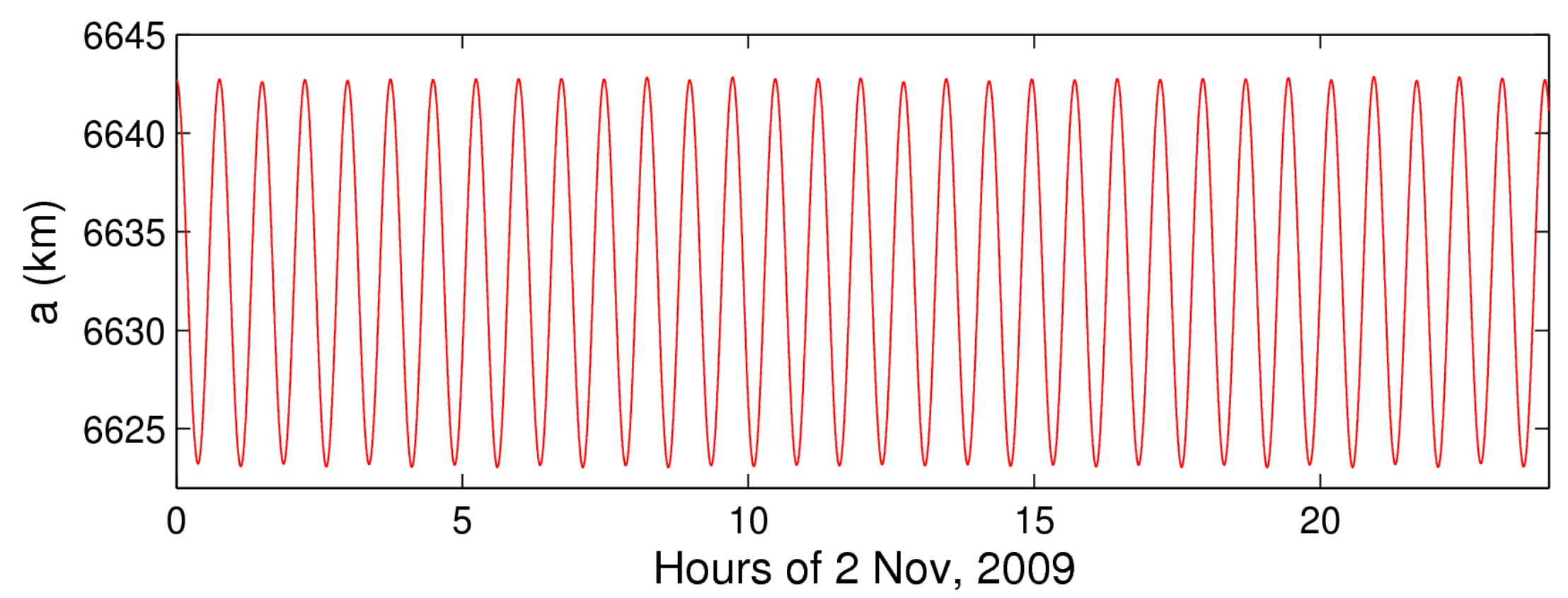

Semi-major axis:

Twice-per-revolution variations of about $\pm 10 \mathrm{~km}$ around the mean semi-major axis of $6632.9 \mathrm{~km}$, which corresponds to a mean altitude of $254.9 \mathrm{~km}$ 


\section{Osculating Orbital Elements of GOCE (3)}

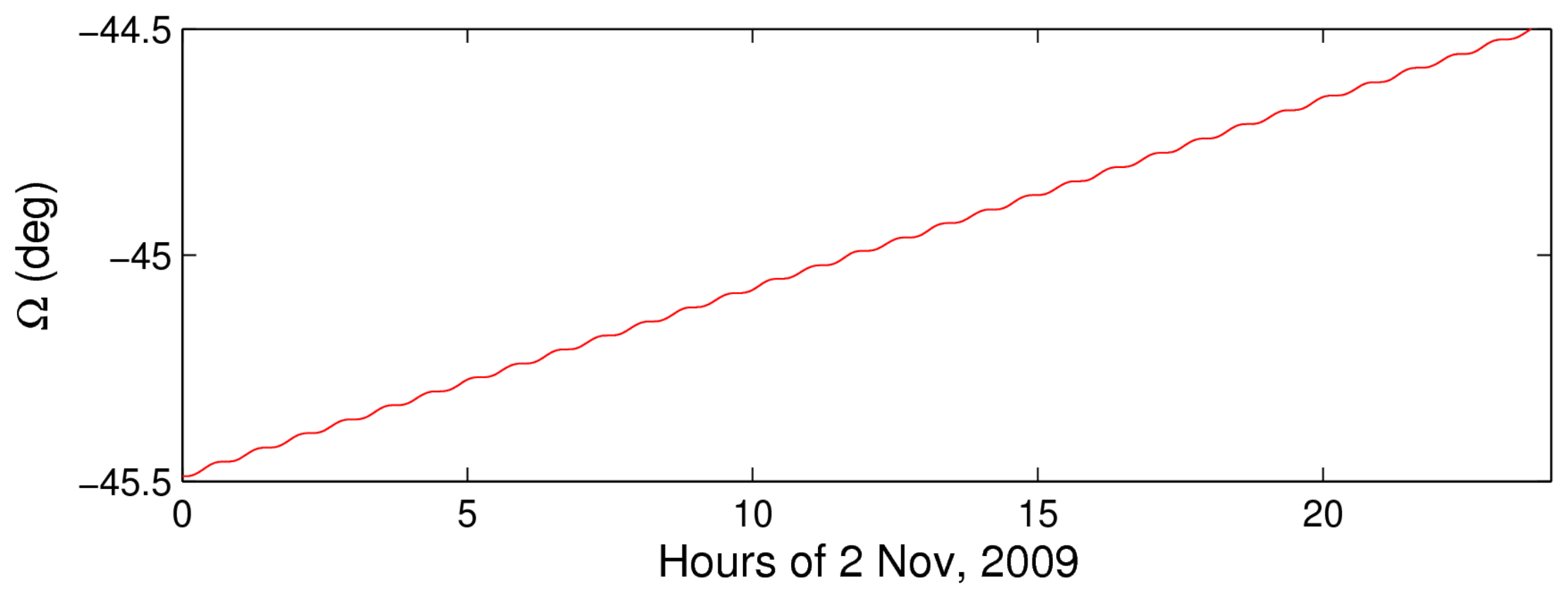

Right ascension of ascending node:

Twice-per-revolution variations and linear drift of about $+1^{\circ} /$ day (360\% 365 days) due to the sun-synchronous orbit 


\section{Dynamic Orbit Representation (3)}

Dynamic orbit positions may be computed at any epoch within the arc

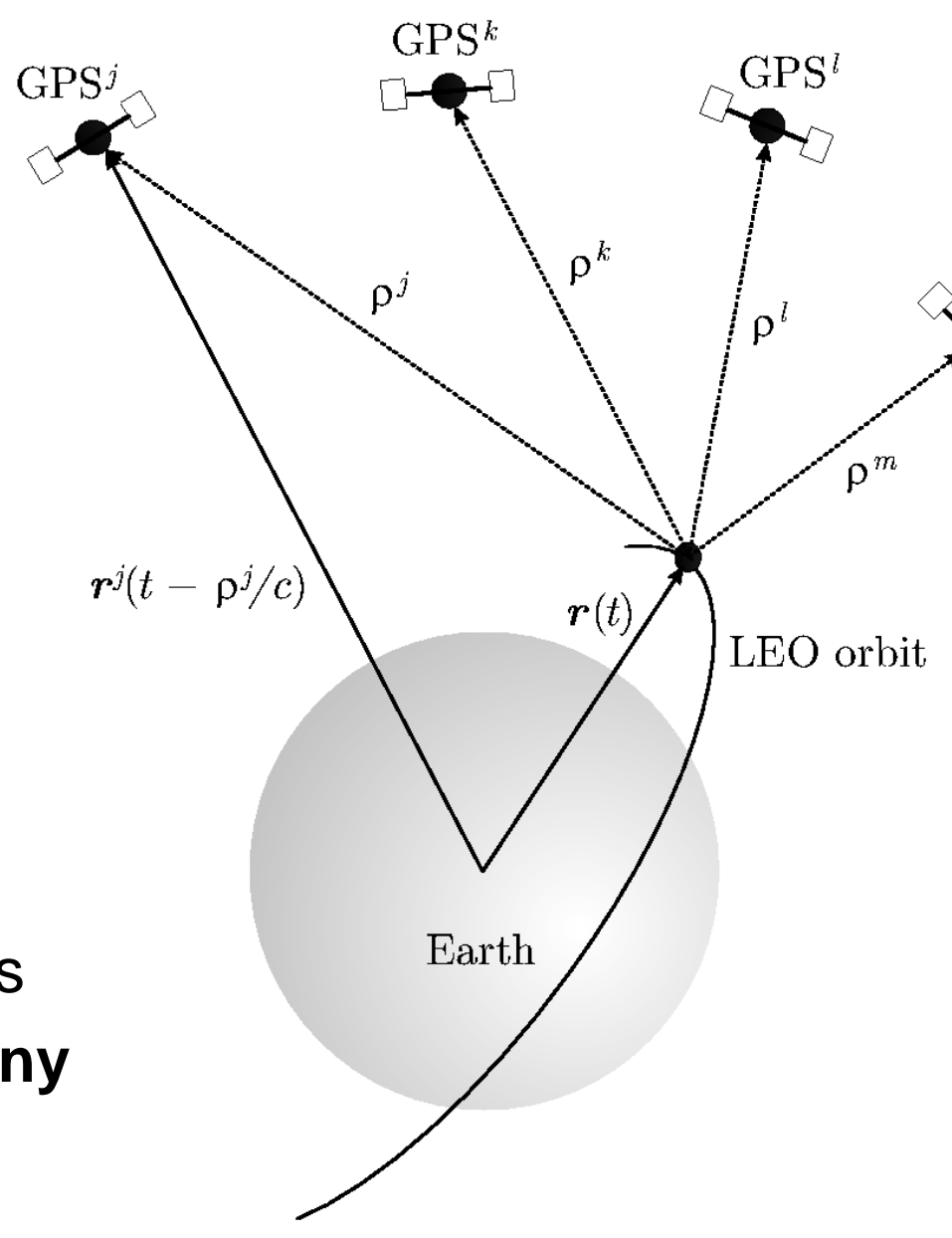

Dynamic positions are fully dependent on the force models used, e.g., on the gravity field model 


\section{Reduced-Dynamic Orbit Representation (1)}

Equation of motion (in inertial frame) is given by:

$$
\ddot{\boldsymbol{r}}=-G M \frac{\boldsymbol{r}}{r^{3}}+\boldsymbol{f}_{1}\left(t, \boldsymbol{r}, \dot{\boldsymbol{r}}, Q_{1}, \ldots, Q_{d}, P_{1}, \ldots, P_{s}\right)
$$

$P_{1}, \ldots, P_{s}$

Pseudo-stochastic parameters

Pseudo-stochastic parameters are:

- additional empirical parameters characterized by a priori known statistical properties, e.g., by expectation values and a priori variances

- useful to compensate for deficiencies in dynamic models, e.g., deficiencies in models describing non-gravitational accelerations

- often set up as piecewise constant accelerations to ensure that satellite trajectories are continuous and differentiable at any epoch 


\section{Reduced-Dynamic Orbit Representation (2)}

Reduced-dynamic orbits are well suited to compute LEO orbits of highest

\section{quality}

(Jäggi et al.,, 2006; Jäggi, 2007)

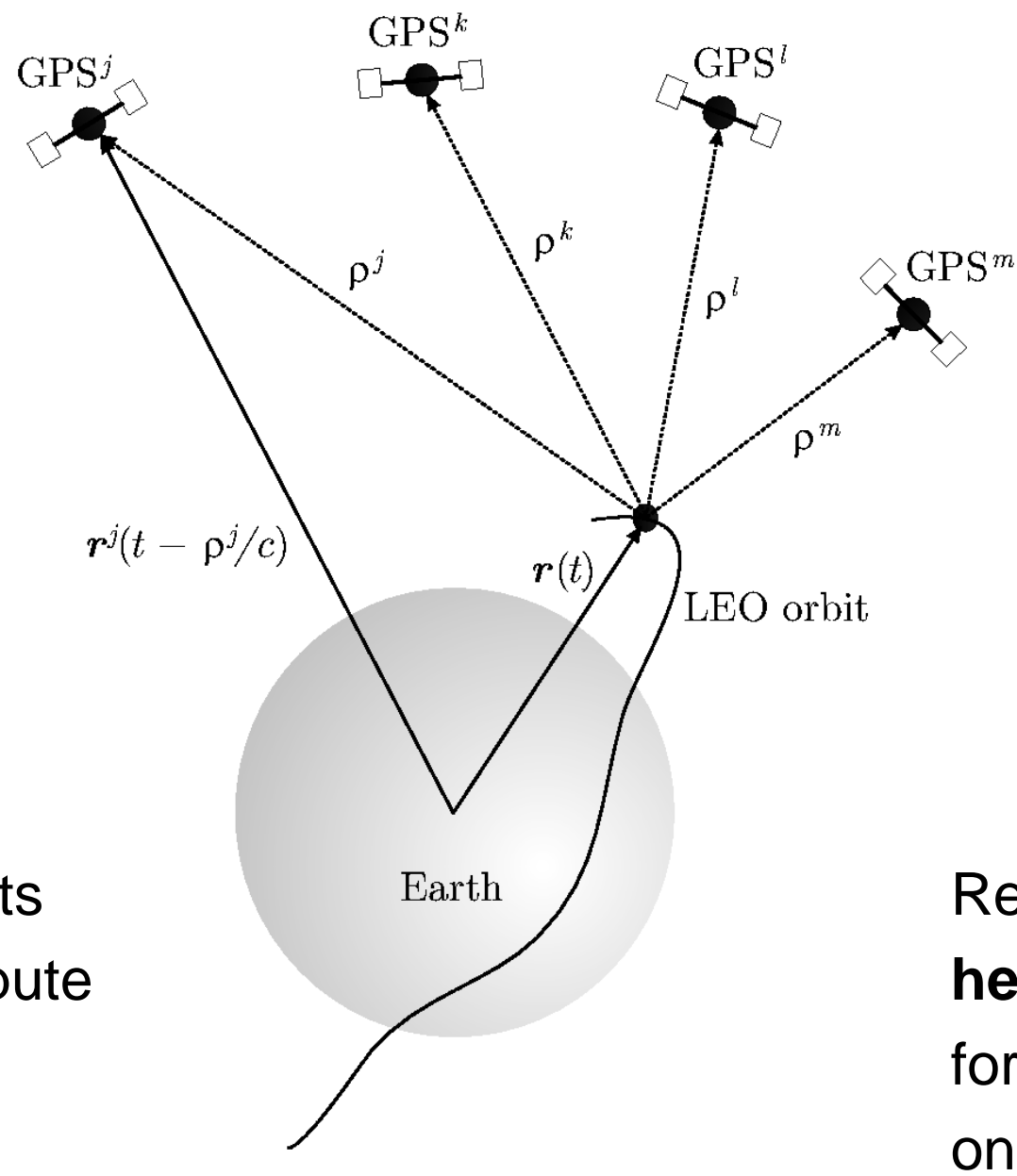

Reduced-dynamic orbits heavily depend on the force models used, e.g., on the gravity field model 


\section{Reduced-dynamic Orbit Representation (3)}

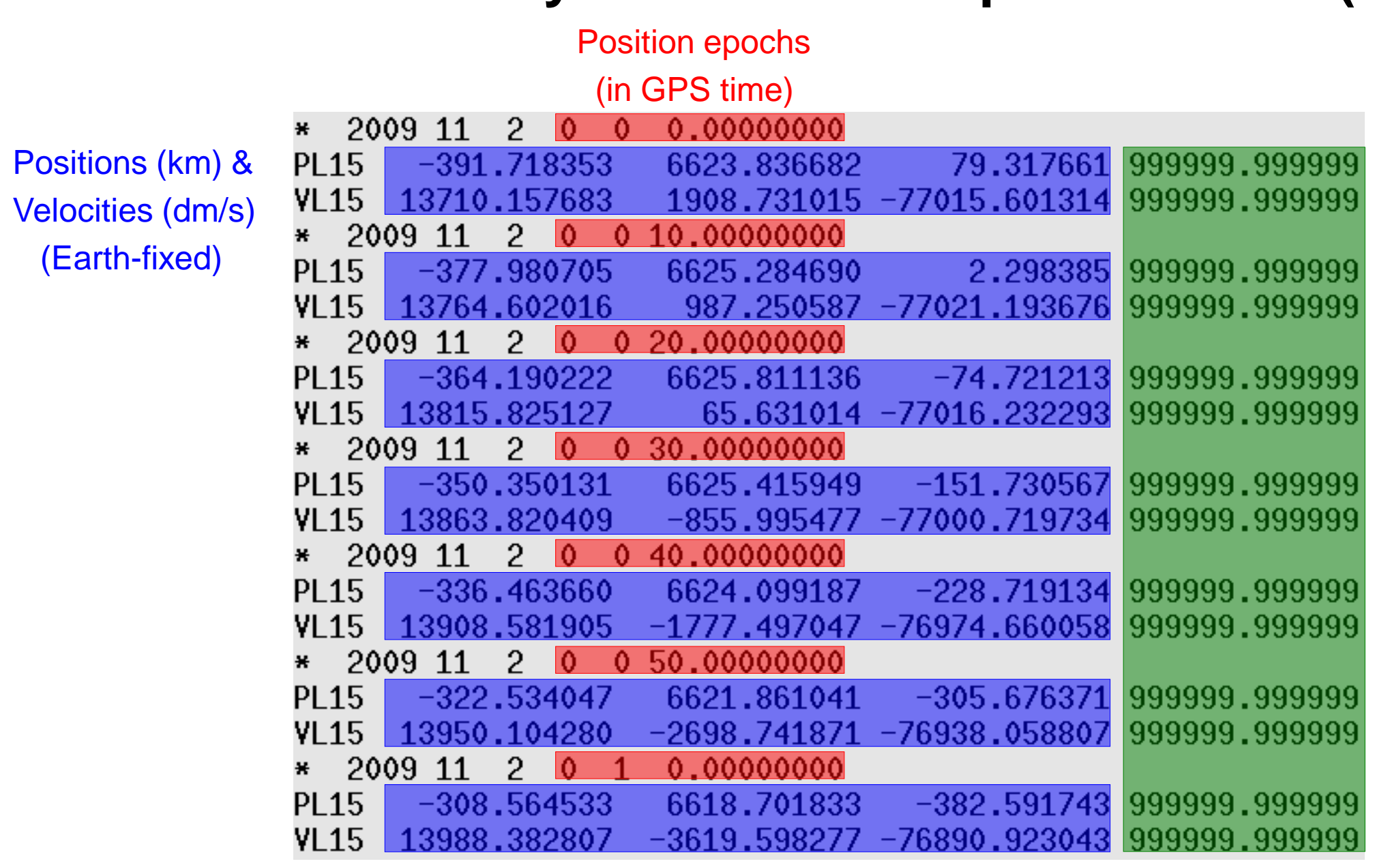

Excerpt of reduced-dynamic GOCE positions at begin of 2 Nov, 2009 GO_CONS_SST_PRD_2_20091101T235945_20091102T235944_0001 


\section{Orbit Determination}




\section{Principle of Orbit Determination}

The actual orbit $\boldsymbol{r}(t)$ is expressed as a truncated Taylor series:

$$
\boldsymbol{r}(t)=\boldsymbol{r}_{0}(t)+\sum_{i=1}^{n} \frac{\partial \boldsymbol{r}_{0}}{\partial P_{i}}(t) \cdot\left(P_{i}-P_{0, i}\right)
$$

$\boldsymbol{r}_{0}(t)$

$\frac{\partial \boldsymbol{r}_{0}}{\partial P_{i}}(t)$

$P_{0, i}$

$P_{i}$
A priori orbit

Partial derivative of the a priori orbit $\boldsymbol{r}_{0}(t)$ w.r.t. parameter $P_{i}$

A priori parameter values of the a priori orbit $\boldsymbol{r}_{0}(t)$

Parameter values of the improved orbit $\boldsymbol{r}(t)$

A least-squares adjustment of spacecraft tracking data yields corrections to the a priori parameter values $P_{0, i}$. Using the above equation, the improved (linearized) orbit $\boldsymbol{r}(t)$ may be eventually computed. 


\section{A priori orbit generation: Keplerian Orbit}

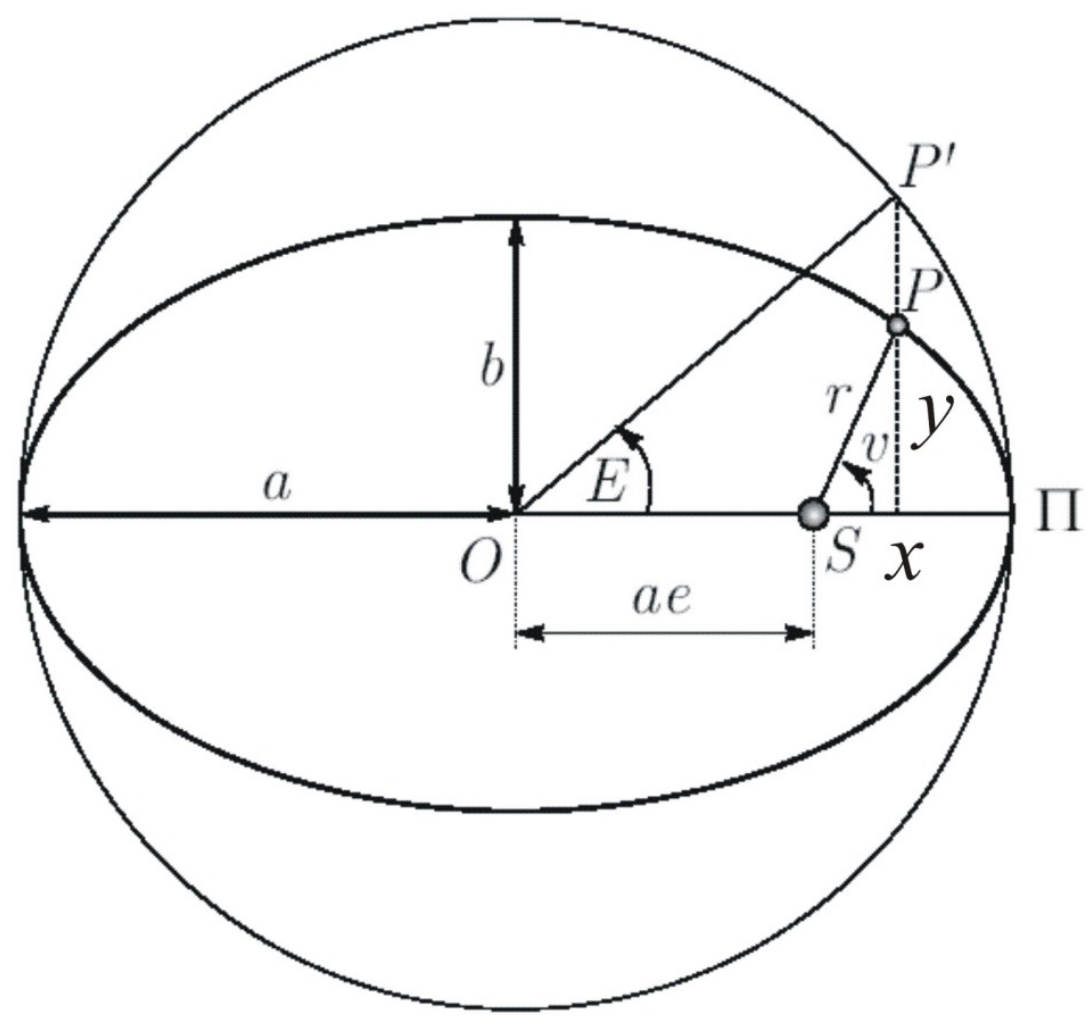

Coordinates in orbital system:

$$
\begin{gathered}
n=\text { mean motion } \\
n^{2} a^{3}=G M \\
M=\text { mean anomaly } \\
M(\mathrm{t})=n\left(t-T_{0}\right)
\end{gathered}
$$

Kepler's equation:

$E=$ eccentric anomaly

$$
\begin{gathered}
E(\mathrm{t})=M(\mathrm{t})+e \sin E(\mathrm{t}) \\
x=a(\cos E-e) \\
y=a \sqrt{1-e^{2}} \sin E
\end{gathered}
$$




\section{A priori orbit generation: Keplerian Orbit}

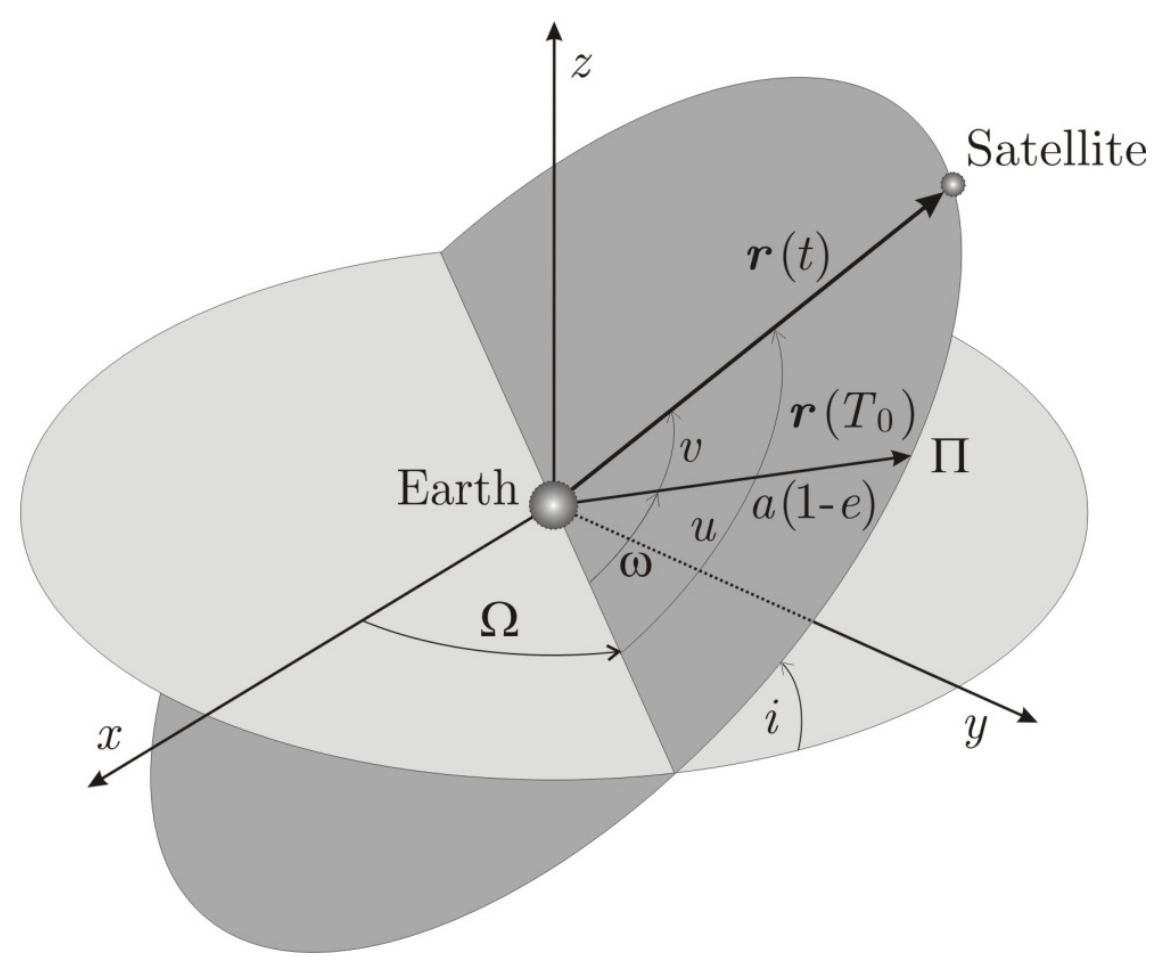

The resulting formulas are those used in the two-body problem for ephemerides calculations. They are coded in the SR ephem which is used for the exercises.

\section{Positions in equatorial system:}

They follow from the coordinates in the orbital system by adopting three particular rotations:

$$
\left(\begin{array}{c}
x_{a} \\
y_{a} \\
z_{a}
\end{array}\right)=\mathbf{R}_{3}(-\Omega) \cdot \mathbf{R}_{1}(-i) \cdot \mathbf{R}_{3}(-\omega) \cdot\left(\begin{array}{l}
x \\
y \\
0
\end{array}\right)
$$

The same holds for the velocities:

$$
\left(\begin{array}{c}
\dot{x}_{a} \\
\dot{y}_{a} \\
\dot{z}_{a}
\end{array}\right)=\mathbf{R}_{3}(-\Omega) \cdot \mathbf{R}_{1}(-i) \cdot \mathbf{R}_{3}(-\omega) \cdot\left(\begin{array}{c}
\dot{x} \\
\dot{y} \\
0
\end{array}\right)
$$




\section{Numerical Integration (1)}

Collocation algorithms (one particular class of numerical integration techniques) are subsequently used to briefly illustrate the principles of numerical integration:

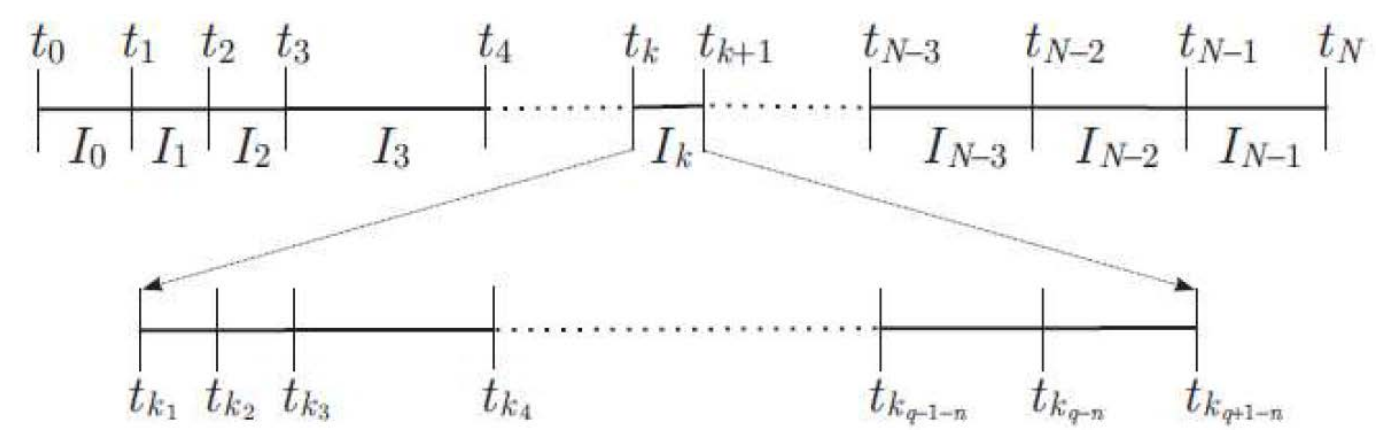

The original intervall is divided into $N$ integration intervals. For each interval $I_{k}$ a further subdivision is performed according to the order $q$ of the adopted method. At these points $t_{k_{j}}$ the numerical solution is requested to solve the differential equation system of order $n$.

(Beutler, 2005) 


\section{Numerical Integration (2)}

Initial value problem in the interval $I_{k}$ is given by:

$$
\ddot{\mathbf{r}}_{k}=\mathbf{f}\left(t, \mathbf{r}_{k}, \dot{\mathbf{r}}_{k}\right)
$$

with initial conditions

$$
\mathbf{r}_{k}\left(t_{k}\right) \doteq \mathbf{r}_{k 0} \quad \text { and } \quad \dot{\mathbf{r}}_{k}\left(t_{k}\right) \doteq \dot{\mathbf{r}}_{k 0}
$$

where the initial values are defined as

$$
\mathbf{r}_{k 0}^{(i)}= \begin{cases}\mathbf{r}_{0}^{(i)} & ; k=0 \\ \mathbf{r}_{k-1}^{(i)}\left(t_{k}\right) & ; k>0\end{cases}
$$




\section{Numerical Integration (3)}

The collocation method approximates the solution in the interval $I_{k}$ by:

$$
\mathbf{r}_{k}(t) \doteq \sum_{l=0}^{q} \frac{1}{l !}\left(t-t_{k}\right)^{l} \mathbf{r}_{k 0}^{(l)}
$$

The coefficients $\mathbf{r}_{k 0}^{(l)}, l=0, \ldots, q$ are obtained by requesting that the numerical solution assumes the initial values and solves the differential equation system at $q-1$ different epochs $t_{k_{j}}, j=1, \ldots, q-1$. This leads to the conditions

$$
\sum_{l=2}^{q} \frac{\left(t_{k_{j}}-t_{k}\right)^{l-2}}{(l-2) !} \mathbf{r}_{k 0}^{(l)}=\mathbf{f}\left(t_{k_{j}}, \mathbf{r}_{k}\left(t_{k_{j}}\right), \dot{\mathbf{r}}_{k}\left(t_{k_{j}}\right)\right), \quad j=1, \ldots, q-1 .
$$

They are non-linear but can be solved efficiently by an iterative procedure.

(Beutler, 2005) 


\section{Pocket Guide of Least-Squares Adjustment (1)}

The system of Observation Equations is given by:

$$
\boldsymbol{L}^{\prime}+\boldsymbol{\epsilon}=\boldsymbol{F}(\boldsymbol{X})
$$

or, if $\boldsymbol{F}$ is a non-linear function of the parameters, in its linearized form:

$$
\boldsymbol{L}^{\prime}+\boldsymbol{\epsilon}=\boldsymbol{F}\left(\boldsymbol{X}_{0}\right)+\boldsymbol{A} \boldsymbol{x}
$$

$\boldsymbol{L}^{\prime} \quad$ Tracking observations

$\boldsymbol{X}_{0} \quad$ A priori parameter values

$\boldsymbol{\epsilon} \quad$ Observation corrections

$\boldsymbol{x} \quad$ Parameter corrections

$\boldsymbol{F} \quad$ Functional model

$\boldsymbol{X}$ Improved parameter values,

$$
\left.\boldsymbol{A} \doteq \frac{\partial \boldsymbol{F}(\boldsymbol{X})}{\partial \boldsymbol{X}}\right|_{\boldsymbol{X}=\boldsymbol{X}_{0}}
$$

i.e., $\boldsymbol{X}=\boldsymbol{X}_{0}+\boldsymbol{x}$

First design matrix 


\section{Pocket Guide of Least-Squares Adjustment (2)}

The system of Normal Equations is obtained by minimizing $\boldsymbol{\epsilon}^{T} P \boldsymbol{\epsilon}$ :

$$
\left(\boldsymbol{A}^{T} \boldsymbol{P} \boldsymbol{A}\right) \boldsymbol{x}-\boldsymbol{A}^{T} \boldsymbol{P} \boldsymbol{l}=\boldsymbol{N} \boldsymbol{x}-\boldsymbol{b}=\mathbf{0}
$$

$\boldsymbol{N} \doteq \boldsymbol{A}^{T} \boldsymbol{P} \boldsymbol{A} \quad$ Normal equation matrix

$\boldsymbol{b} \doteq \boldsymbol{A}^{T} \boldsymbol{P} \boldsymbol{l} \quad$ Right-hand side with "O-C" term $\boldsymbol{l} \doteq \boldsymbol{L}^{\prime}-\boldsymbol{F}\left(\boldsymbol{X}_{0}\right)$

$\boldsymbol{P}=\sigma_{0}^{2} \boldsymbol{C}_{\boldsymbol{l l}}^{-1} \quad$ Weight matrix, from covariance matrix $\boldsymbol{C}_{\boldsymbol{l l}}$ of observations

For a regular normal equation matrix the parameter corrections follow as:

$$
\boldsymbol{x}=\left(\boldsymbol{A}^{T} \boldsymbol{P} \boldsymbol{A}\right)^{-1} \boldsymbol{A}^{T} \boldsymbol{P} \boldsymbol{l}=\boldsymbol{N}^{-1} \boldsymbol{b}
$$




\section{Pocket Guide of Least-Squares Adjustment (3)}

The a posteriori standard deviation of unit weight is computed as:

$$
m_{0}=\sqrt{\frac{\boldsymbol{\epsilon}^{T} P \boldsymbol{\epsilon}}{f}}
$$

$f \quad$ Degree of freedom (number of observations minus number of parameters)

The covariance matrix of the adjusted parameters is given by

$$
\boldsymbol{C}_{\boldsymbol{x} \boldsymbol{x}}=m_{0}^{2} \boldsymbol{Q}_{\boldsymbol{x} \boldsymbol{x}}=m_{0}^{2} \boldsymbol{N}^{-1}
$$

and their a posteriori standard deviations follow from the diagonal elements:

$$
m_{x}=\sqrt{C_{x x}}=m_{0} \sqrt{Q_{x x}}
$$




\section{Pocket Guide of Least-Squares Adjustment (4)}

Parameter constraints may be introduced by artificial observations with a userspecified variance $\sigma_{a b s}^{2}$. These observations have to be appended to the system of observation equations. If the change with respect to the a priori value is used as the actual parameter in the artificial observation equation, the weight

$$
W=\frac{\sigma_{0}^{2}}{\sigma_{a b s}^{2}}
$$

has to be only added to the corresponding diagonal element of the normal equation matrix $\boldsymbol{N}$, because the value $\mathrm{O}-\mathrm{C}$ is zero in this special case. 


\section{Example of Filter Approaches (1)}

Assuming that measurement data are uncorrelated between measurement epochs, and that the epoch-wise weight matrix is denoted by $P_{j}$, the normal equation system at epoch no. $j$ reads as

$$
\begin{aligned}
& N_{j} \Delta x_{0 j}=b_{j} \\
& N_{j}=\left(A^{T} P A\right)_{j}=\sum_{k=0}^{j} A_{k}^{T} P_{k} A_{k} \doteq \sum_{k=0}^{j} \Delta N_{k} \quad \Delta x_{0 j}=Q_{j} b_{j} \\
& b_{j}=\left(A^{T} P \Delta l\right)_{j}=\sum^{j} A_{k}^{T} P_{k} \Delta l_{k} \doteq \sum^{j} \Delta b_{k} \quad Q_{j}=N_{j}^{-1}
\end{aligned}
$$

The index $\mathrm{j}$ indicates that for the solution all measurements up to epoch $t_{j}$ are used 


\section{Example of Filter Approaches (2)}

The recursion formula for the non-inverted normal equation system is trivial:

$$
\begin{aligned}
& N_{j+1} \Delta x_{0, j+1}=b_{j+1} \\
& N_{j+1}=N_{j}+\Delta N_{j+1} \\
& b_{j+1}=b_{j}+\Delta b_{j+1}
\end{aligned}
$$

Solution vectors, error estimates, and covariance matrices can be computed at every measurement epoch if required. If the dimension of $N_{j}$ is large, however, a frequent inversion will not be a preferred solution strategy. 


\section{Example of Filter Approaches (3)}

A recursion formula can also be derived for the inverted normal equation system:

$$
\begin{aligned}
& N_{j+1} \Delta x_{0, j+1}=b_{j+1} \\
& \Delta x_{0, j+1}=Q_{j+1}\left(b_{j}+\Delta b_{j+1}\right)=Q_{j+1}\left(N_{j} \Delta x_{0 j}+\Delta b_{j+1}\right) \\
& \Delta x_{0, j+1}=Q_{j+1}\left(\left\{N_{j+1}-\Delta N_{j+1}\right\} \Delta x_{0 j}+\Delta b_{j+1}\right) \\
& \Delta x_{0, j+1}=\Delta x_{0 j}+Q_{j+1} A_{j+1}^{T} P_{j+1}\left\{\Delta l_{j+1}-A_{j+1} \Delta x_{0 j}\right\} \\
& \Delta x_{0, j+1}=\Delta x_{0 j}+K_{j+1}\left\{\Delta l_{j+1}-A_{j+1} \Delta x_{0 j}\right\}
\end{aligned}
$$

The formulas are well suited for real-time applications as it is straightforward to check the results for plausibility. They are also closely related to the Kalman filter formulas. Aspects not presented here are the transformation of the parameters to a new set of parameters at every epoch and stochastic system equations. 


\section{Example of Filter Approaches (4)}
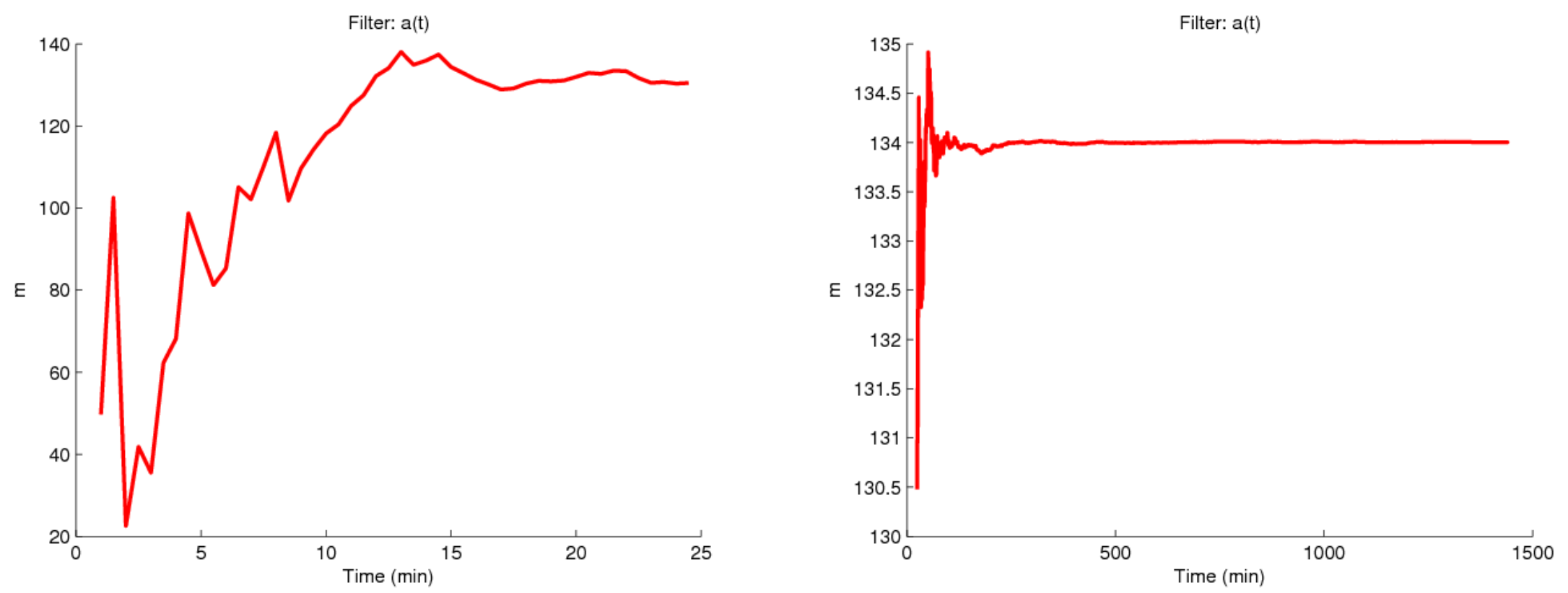

Example from the exercise: Semi-major axis refering to $t_{0}$ from a filter solution. Left: epochs $3-50$, Right: epochs 50 until end of the day. Even with only a few epochs the solution is much better than the "quick and dirty" polynomial fit. After about 500 min the solution is stable. 


\section{Example of Filter Approaches (5)}

For the recursion formula developed so far the following relations may be used:

$$
N_{j+1}=N_{j}+\Delta N_{j+1}, \quad Q_{j+1}=\left(N_{j+1}\right)^{-1}
$$

If the dimension of $N_{j+1}$ is large, the following recursion formula, following from a rather laborious derivation, is preferred:

$$
\begin{aligned}
Q_{j+1} & =Q_{j}-Q_{j} A_{j+1}^{T} P_{j+1}\left(E+A_{j+1} Q_{j} A_{j+1}^{T} P_{j+1}\right)^{-1} A_{j+1} Q_{j} \\
Q_{j+1} & =Q_{j}-Q_{j} A_{j+1}^{T}\left(P_{j+1}^{-1}+A_{j+1} Q_{j} A_{j+1}^{T}\right)^{-1} A_{j+1} Q_{j}
\end{aligned}
$$

The matrix to be inverted is of the size of the number of measurements to be processed per epoch. 


\section{Partial Derivatives}

The partial of the $r$-th observation w.r.t. orbit parameter $P_{i}$ may be expressed as

$$
\frac{\partial F_{r}(\boldsymbol{X})}{\partial P_{i}}=\left(\boldsymbol{\nabla}\left(F_{r}(\boldsymbol{X})\right)\right)^{T} \cdot \frac{\partial \boldsymbol{r}_{0}}{\partial P_{i}}(t)
$$

with the gradient given by

$$
\left(\boldsymbol{\nabla}\left(F_{r}(\boldsymbol{X})\right)\right)^{T}=\left(\frac{\partial F_{r}(\boldsymbol{X})}{\partial r_{0,1}} \frac{\partial F_{r}(\boldsymbol{X})}{\partial r_{0,2}} \frac{\partial F_{r}(\boldsymbol{X})}{\partial r_{0,3}}\right)
$$

if the observations only depend on the geocentric position vector and are referring to only one epoch. The gradient only depends on the type of observations used, whereas the second term is independent of the observation type and related to the variational equations. This separates the observation-specific (geometric) part from the dynamic part. 


\section{Variational Equations (1)}

For each orbit parameter $P_{i}$ the corresponding variational equation reads as

$$
\ddot{\boldsymbol{z}}_{P_{i}}=\boldsymbol{A}_{0} \cdot \boldsymbol{z}_{P_{i}}+\boldsymbol{A}_{1} \cdot \dot{\boldsymbol{z}}_{P_{i}}+\frac{\partial \boldsymbol{f}_{1}}{\partial P_{i}}
$$

with the $3 \times 3$ matrices defined by

$$
A_{0[i ; k]} \doteq \frac{\partial f_{i}}{\partial r_{0, k}} \quad \text { and } \quad A_{1[i ; k]} \doteq \frac{\partial f_{i}}{\partial \dot{r}_{0, k}}
$$

$f_{i} \quad i$-th component of the total acceleration $\boldsymbol{f}$

$r_{0, k} \quad k$-th component of the geocentric position $\boldsymbol{r}_{0}$

For each orbit parameter $P_{i}$ the variational equation is a linear differential equation system of second order in time. Their solutions are all needed for orbit determination. 


\section{Variational Equations (2)}

The variational equation is a linear, homogeneous system with initial values

$$
\boldsymbol{z}_{P_{i}}\left(t_{0}\right) \neq \mathbf{0} \quad \text { and } \quad \dot{\boldsymbol{z}}_{P_{i}}\left(t_{0}\right) \neq \mathbf{0} \quad \text { for } \quad P_{i} \in\left\{a, e, i, \Omega, \omega, u_{0}\right\}
$$

and a linear, inhomogeneous system with initial values

$$
\boldsymbol{z}_{P_{i}}\left(t_{0}\right)=\mathbf{0} \quad \text { and } \quad \dot{\boldsymbol{z}}_{P_{i}}\left(t_{0}\right)=\mathbf{0} \quad \text { for } \quad P_{i} \in\left\{Q_{1}, \ldots, Q_{d}\right\}
$$

Let us assume that the functions $z_{O_{j}}(t), j=1, \ldots, 6$ are the partials w.r.t. the six parameters $O_{j}, j=1, \ldots, 6$ defining the initial conditions at time $t_{0}$. The ensemble of these six functions forms one complete system of solutions of the homogeneous part of the variational equation, which allows to obtain the solution of the inhomogeneous system by the method of "variation of constants". 


\section{Variational Equations (3)}

The solution and its first time derivative may be written as

$$
\boldsymbol{z}_{P_{i}}^{(k)}(t)=\sum_{j=1}^{6} \alpha_{O_{j} P_{i}}(t) \cdot \boldsymbol{z}_{O_{j}}^{(k)}(t) ; \quad k=0,1
$$

with the coefficient functions defined by

$$
\boldsymbol{\alpha}_{P_{i}}(t) \doteq \int_{t_{0}}^{t} \boldsymbol{Z}^{-1}\left(t^{\prime}\right) \cdot \boldsymbol{h}_{P_{i}}\left(t^{\prime}\right) \cdot d t^{\prime}
$$

$\boldsymbol{\alpha}_{P_{i}} \quad$ column array defined by $\left(\alpha_{O_{1} P_{i}}, \ldots, \alpha_{O_{6} P_{i}}\right)^{T}$

$\boldsymbol{Z} \quad 6 \times 6$ matrix defined by $\boldsymbol{Z}_{[1, \ldots, 3 ; j]} \doteq \boldsymbol{z}_{O_{j}}, \boldsymbol{Z}_{[4, \ldots, 6 ; j]} \doteq \dot{\boldsymbol{z}}_{O_{j}}$

$\boldsymbol{h}_{P_{i}} \quad$ column array defined by $\left(\mathbf{0}^{T}, \partial \boldsymbol{f}_{1}^{T} / \partial P_{i}\right)^{T}$ 


\section{Variational Equations (4)}

Note that the solutions $\boldsymbol{z}_{P_{i}}(t)$ of the variational equation and its time derivative may be expressed with the same functions $\alpha_{O_{j} P_{i}}(t)$ as a linear combination with the homogeneous solutions $\boldsymbol{z}_{O_{j}}(t)$ and $\dot{\boldsymbol{z}}_{O_{j}}(t)$, respectively. Therefore, only the six initial value problems associated with the initial conditions have to be actually treated as differential equation systems. Their solutions have to be either obtained approximately, or by numerical integration techniques.

All variational equations related to dynamical orbit parameters may be reduced to definite integrals. They can be efficiently solved numerically, e.g., by a Gaussian quadrature technique.

It must be emphasized that each additional orbit parameter requires an additional numerical solution of a definite integral. In view of the potentially large number of orbit parameters, it is advantagous that for pseudo-stochastic orbit parameters an explicit numerical quadrature of the definite integrals can be avoided.

(Jäggi, 2007)

\section{$\boldsymbol{u}^{b}$}




\section{Partial Derivatives for Keplerian Orbits}

The partial derivatives wrt the orbital elements can be explicitly derived for Keplerian orbits by using the formulas of the two-body problem:

$$
\left(\begin{array}{l}
x_{e} \\
y_{e} \\
z_{e}
\end{array}\right)=\mathbf{R}_{3}(-\Omega) \cdot \mathbf{R}_{1}(-i) \cdot \mathbf{R}_{3}(-\omega) \cdot\left(\begin{array}{l}
a(\cos E-e) \\
a \sqrt{1-e^{2}} \cdot \sin E \\
0
\end{array}\right)
$$

As an example, for the partial derivative wrt the inclination $i$ we obtain

$$
\frac{\partial}{\partial \mathrm{i}}\left(\begin{array}{l}
x_{e} \\
y_{e} \\
z_{e}
\end{array}\right)=\mathbf{R}_{3}(-\Omega) \cdot \frac{\partial}{\partial i}\left\{\mathbf{R}_{1}(-i)\right\} \cdot \mathbf{R}_{3}(-\omega) \cdot\left(\begin{array}{l}
a(\cos E-e) \\
a \sqrt{1-e^{2}} \cdot \sin E \\
0
\end{array}\right)
$$

Similar expressions are also obtained for the other partial derivatives. Note that the partials wrt $a$ and $e$ are more complicated as $E$ depends on them, as well. 


\section{Pulses (1)}

The special case of instantaneous velocity changes (pulses) $V_{i}$ at times $t_{i}$ in predetermined directions $\boldsymbol{e}\left(t_{i}\right)$ is particularly simple. The contribution of this parameter $P_{i}=V_{i}$ to $\boldsymbol{f}_{1}$ may be formally written as $V_{i} \cdot \delta\left(t-t_{i}\right) \cdot \boldsymbol{e}(t)$ and the corresponding variational equation reads as

$$
\ddot{z}_{V_{i}}=\boldsymbol{A}_{0} \cdot \boldsymbol{z}_{V_{i}}+\boldsymbol{A}_{1} \cdot \dot{\boldsymbol{z}}_{V_{i}}+\delta\left(t-t_{i}\right) \cdot \boldsymbol{e}(t)
$$

The coefficients $\boldsymbol{\alpha}_{V_{i}}(t)$ read for this special case as

$$
\boldsymbol{\alpha}_{V_{i}}(t) \doteq \int_{t_{0}}^{t} \delta\left(t^{\prime}-t_{i}\right) \cdot \boldsymbol{Z}^{-1}\left(t^{\prime}\right) \cdot \boldsymbol{h}_{V_{i}}\left(t^{\prime}\right) \cdot d t^{\prime}=\boldsymbol{Z}^{-1}\left(t_{i}\right) \cdot \boldsymbol{h}_{V_{i}}\left(t_{i}\right) \doteq \beta_{V_{i}}
$$

Subsequently, an alternative, more intuitive derivation is given. 


\section{Pulses (2)}

Let us assume that we want to allow for an instantaneous velocity change of the orbit $\boldsymbol{r}(t)$ at the epoch $t_{i}$ in the direction of the unit vector $\boldsymbol{e}$. We want the resulting orbit to be continuous. The difference of the new - old orbit at $t_{i}$ obviously is given for $t=t_{i}$ by:

$$
\begin{aligned}
& \delta \dot{\boldsymbol{r}}\left(t_{i}\right)=\delta v \boldsymbol{e} \\
& \delta \boldsymbol{r}\left(t_{i}\right)=\mathbf{0} .
\end{aligned}
$$

The difference of the new - old orbit for $t \geq t_{i}$ obviously is given by:

$$
\delta \boldsymbol{r}(t)=\left(\frac{\partial \boldsymbol{r}}{\partial(\delta v)}\right)(t) \delta v
$$




\section{Pulses (2)}

where

$$
\begin{aligned}
& \left(\frac{\partial \boldsymbol{r}}{\partial(\delta v)}\right)\left(t_{i}\right)=\mathbf{0} \\
& \left(\frac{\partial \dot{\boldsymbol{r}}}{\partial(\delta v)}\right)\left(t_{i}\right)=\boldsymbol{e}
\end{aligned}
$$

As the partial derivative is a solution of the homogeneous variational equations, we may write

$$
\left(\frac{\partial \boldsymbol{r}}{\partial(\delta v)}\right)(t)=\sum_{k=1}^{6} \beta_{k}\left(\frac{\partial \boldsymbol{r}}{\partial I_{k}}\right)(t) \stackrel{\text { def }}{=} \sum_{k=1}^{6} \beta_{k} \boldsymbol{z}_{k}(t)
$$

The time independent coefficients $\beta_{k}$ still have to be determined. 


\section{Pulses (3)}

To solve for the unknown coefficients, the linear combination of the six partial derivatives w.r.t. the osculating elements at time $t_{0}$ have to be inserted into the equations defining the partial derivatives w.r.t. $\delta v$ at time $t_{i}$ :

$$
\begin{aligned}
& \sum_{k=1}^{6} \beta_{k} z_{k}\left(t_{i}\right)=\mathbf{0} \\
& \sum_{k=1}^{6} \beta_{k} \dot{z}_{k}\left(t_{i}\right)=e
\end{aligned}
$$

There is one set of coefficients for each pulse. Even if a huge number of pulses are introduced, there is no necessity to solve additional variational equations. 


\section{Short-Arc Approach}

It is as well possible to not only allow for a discontinuous velocity vector, but also for a discontinuous position vector by setting up instantaneous position changes in addition. In analogy to the instantaneous velocity changes, the coefficients of the linear combination of the six partial derivatives w.r.t. the osculating elements at time $t_{0}$ may be found by solving a system of linear equations:

$$
\begin{gathered}
\text { Position changes: } \\
\sum_{j=1}^{6} \beta_{O_{j} X_{i}} \cdot \mathbf{z}_{O_{j}}\left(t_{i}\right)=\mathbf{e}\left(t_{i}\right) \\
\sum_{j=1}^{6} \beta_{O_{j} X_{i}} \cdot \dot{\mathbf{z}}_{O_{j}}\left(t_{i}\right)=\mathbf{0}
\end{gathered}
$$$$
\text { Velocity changes: }
$$$$
\sum_{j=1}^{6} \beta_{O_{j} V_{i}} \cdot \mathbf{z}_{O_{j}}\left(t_{i}\right)=\mathbf{0}
$$$$
\sum_{j=1}^{6} \beta_{O_{j} V_{i}} \cdot \dot{\mathbf{z}}_{O_{j}}\left(t_{i}\right)=\mathbf{e}\left(t_{i}\right)
$$ 


\section{GPS-based LEO POD}




\section{LEO Sensor Offsets}

Phase center offsets $\delta \boldsymbol{r}_{\text {leo,ant }}$ :

- are needed in the inertial or Earth-fixed frame and have to be transformed from the satellite frame using attitude data from the star-trackers

- consist of a frequency-independent instrument offset, e.g., defined by the center of the instrument's mounting plane (CMP) in the satellite frame

- consist of frequency-dependent phase center offsets (PCOs), e.g., defined wrt the center of the instrument's mounting plane in the antenna frame (ARF)

- consist of frequency-dependent phase center variations (PCVs) varying with the direction of the incoming signal, e.g., defined wrt the PCOs in the antenna frame 


\section{Example: GOCE Sensor Offsets (1)}

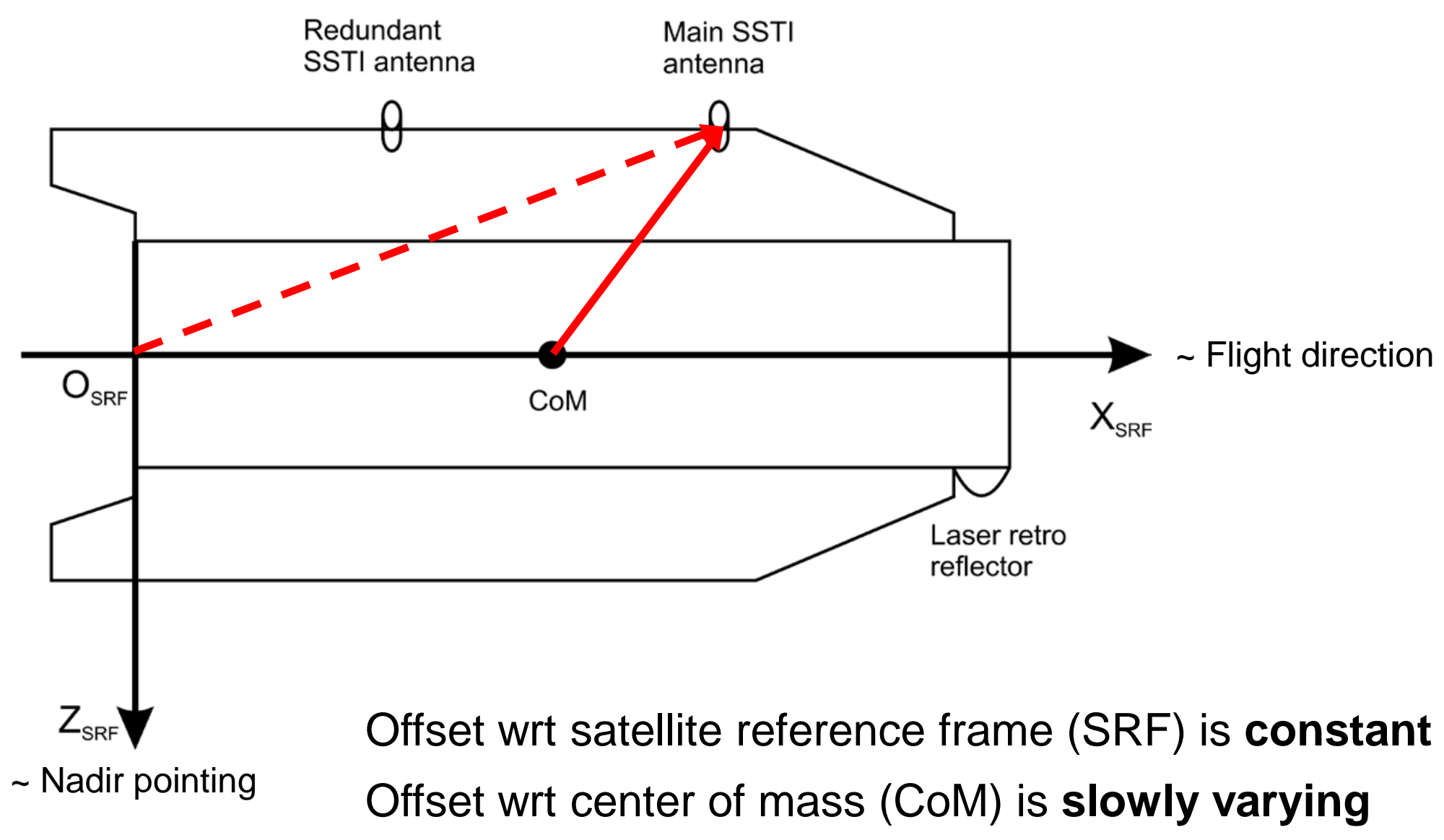




\section{Example: GOCE Sensor Offsets (2)}

Table 1: CoM coordinates in SRF system

\begin{tabular}{|l|r|r|r|}
\hline CoM & $X_{\text {SRF }}[\mathrm{m}]$ & $Y_{\text {SRF }}[\mathrm{m}]$ & $Z_{\text {SRF }}[\mathrm{m}]$ \\
\hline Begin of Life (BoL) & 2.4990 & 0.0036 & 0.0011 \\
\hline End of Life (EoL) & 2.5290 & 0.0038 & 0.0012 \\
\hline
\end{tabular}

Table 2: SSTI antenna CMP coordinates in SRF system

\begin{tabular}{|l|r|r|r|}
\hline CMP coordinates & $X_{\text {SRF }}[\mathrm{m}]$ & $\mathrm{Y}_{\mathrm{SRF}}[\mathrm{m}]$ & $\mathrm{Z}_{\mathrm{SRF}}[\mathrm{m}]$ \\
\hline Main & 3.1930 & 0.0000 & -1.0922 \\
\hline Redundant & 1.3450 & 0.0000 & -1.0903 \\
\hline
\end{tabular}

Table 3: SSTI antenna CMP coordinates wrt to CoM (BoL)

\begin{tabular}{|l|r|r|r|}
\hline CMP coordinates & $X_{\text {CoM }}[\mathrm{m}]$ & $Y_{\text {CoM }}[\mathrm{m}]$ & $Z_{\text {CoM }}[\mathrm{m}]$ \\
\hline Main & 0.6940 & -0.0036 & -1.0933 \\
\hline Redundant & -1.1540 & -0.0036 & -1.0914 \\
\hline
\end{tabular}

Table 4: SSTI antenna phase center offsets in ARF system

\begin{tabular}{|l|r|r|r|}
\hline Phase center offsets & $\mathrm{X}_{\mathrm{ARF}}[\mathrm{mm}]$ & $\mathrm{Y}_{\mathrm{ARF}}[\mathrm{mm}]$ & $\mathrm{Z}_{\mathrm{ARF}}[\mathrm{mm}]$ \\
\hline Main: L1 & -0.18 & 3.51 & -81.11 \\
\hline Main: L2 & -1.22 & -1.00 & -84.18 \\
\hline Redundant: L1 & -0.96 & 3.14 & -81.33 \\
\hline Redundant: L2 & -1.48 & -1.20 & -84.18 \\
\hline
\end{tabular}

Derived from Bigazzi and Frommknecht (2010) 


\section{Example: GOCE GPS Antenna}

\section{L1, L2, Lc phase center offsets}

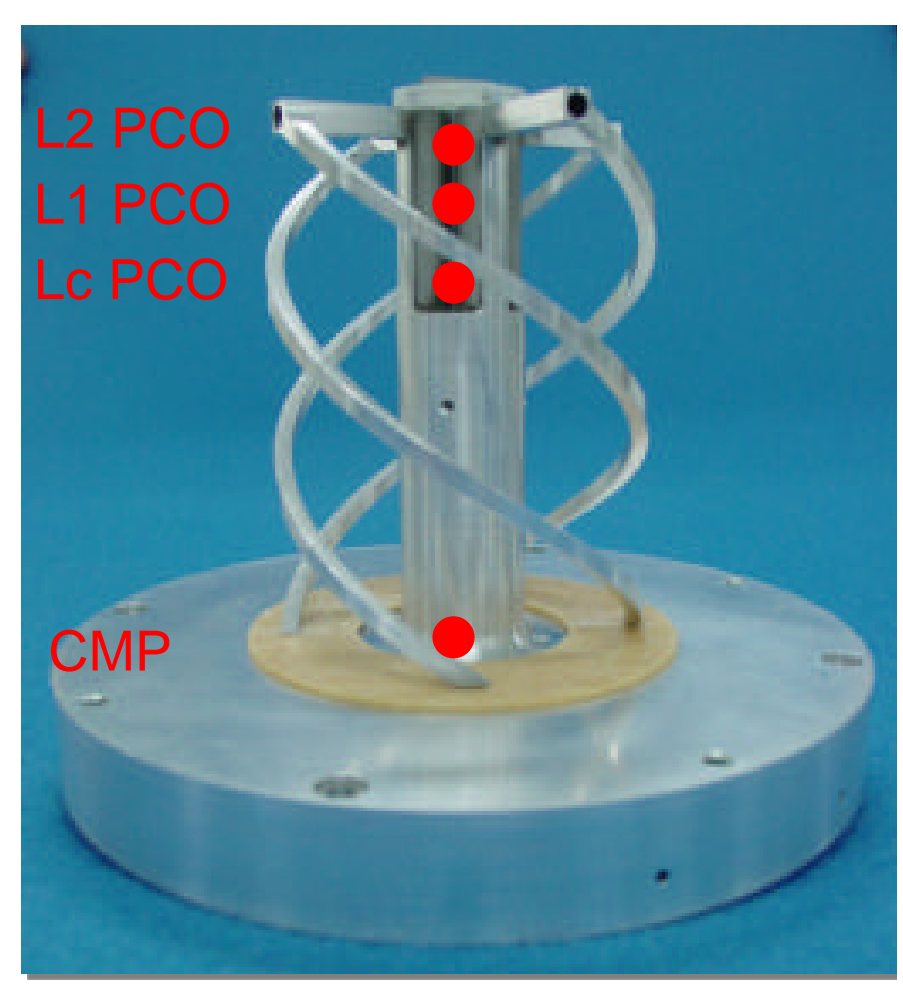

Measured from ground calibration in anechoic chamber

\section{Lc phase center variations}

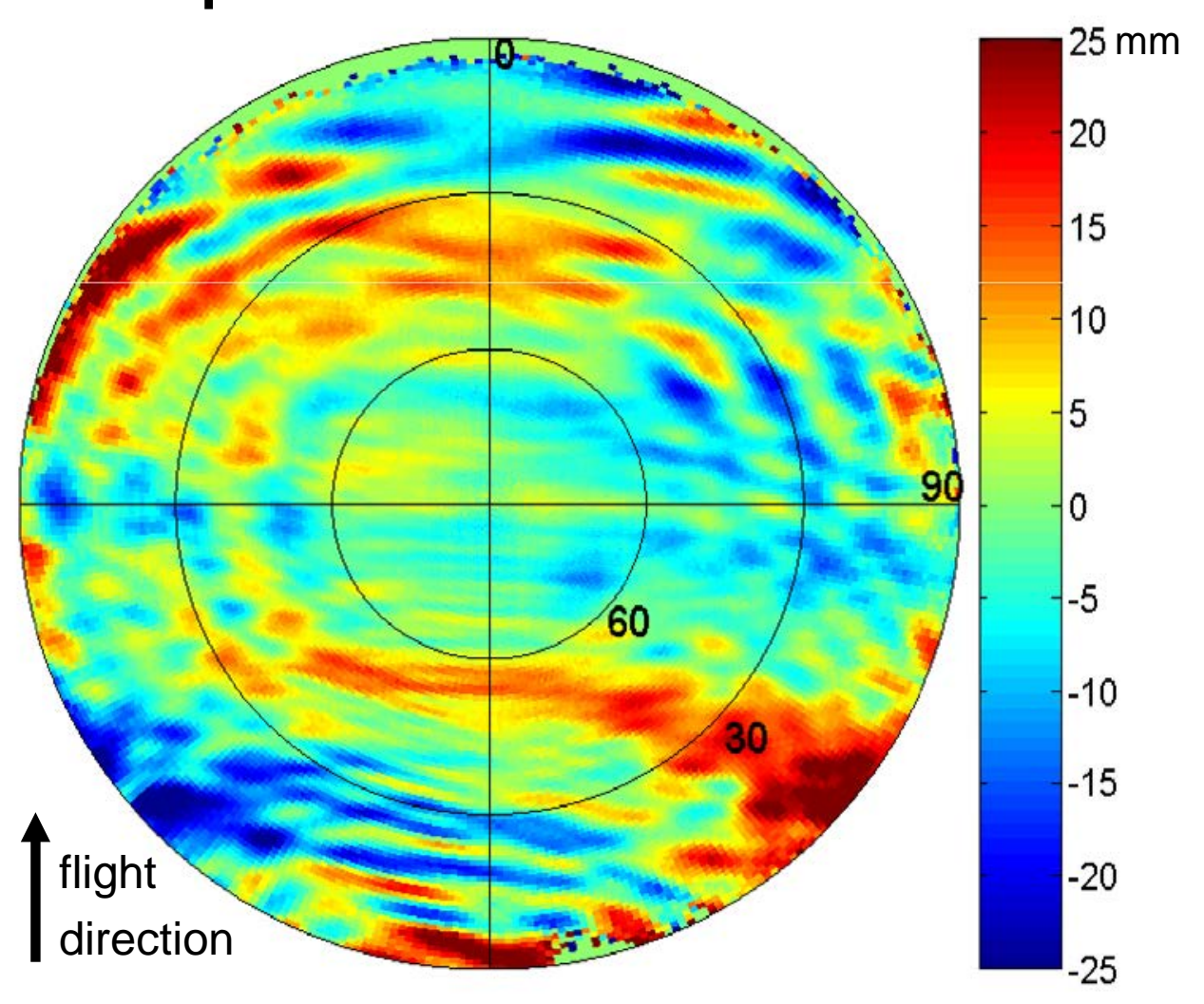

Empirically derived during orbit determination according to Jäggi et al. (2009) 


\section{Visualization of Orbit Solutions}

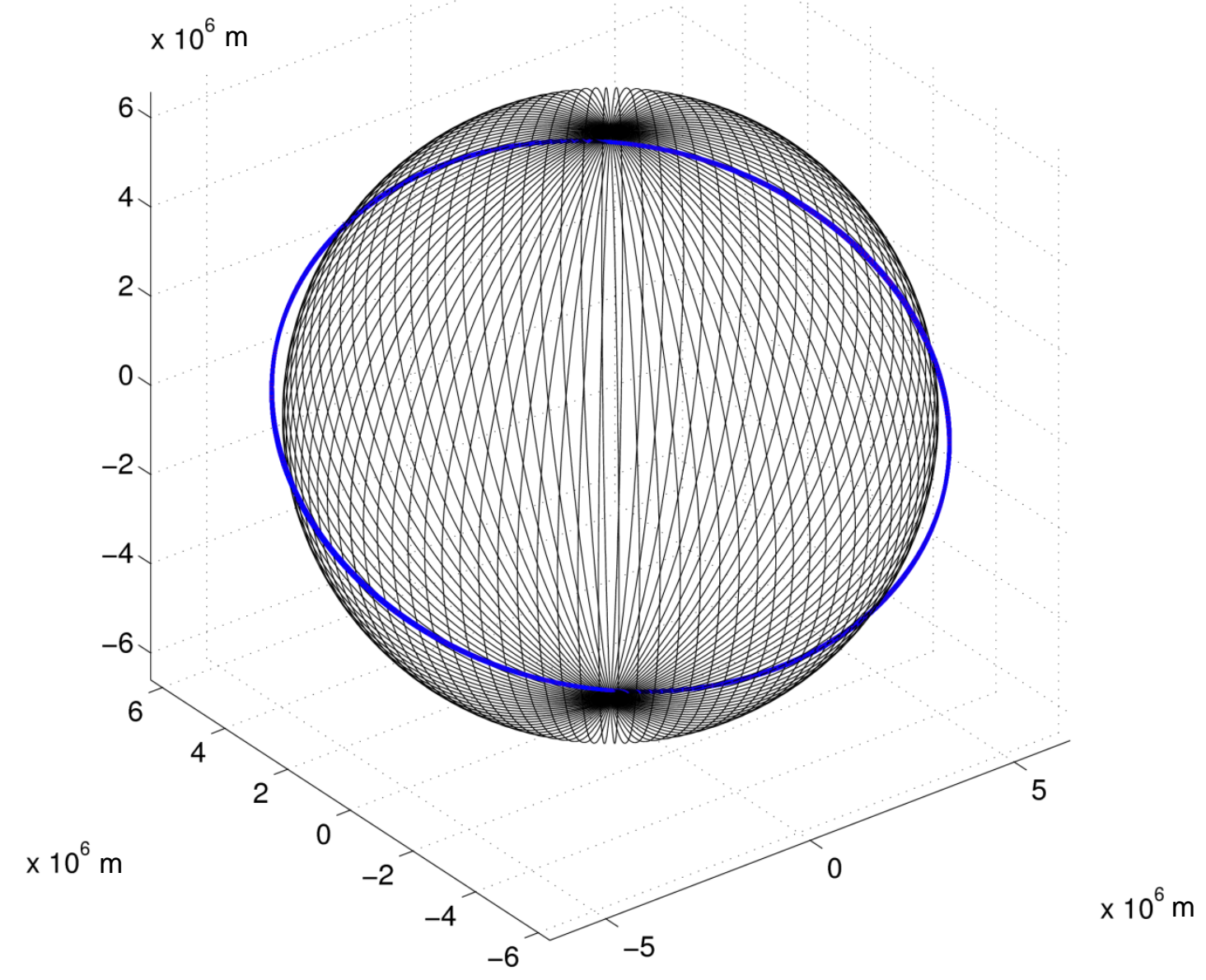

It is more instructive to look at differences between orbits in well suited coordinate systems ... 


\section{Co-Rotating Orbital Frames}

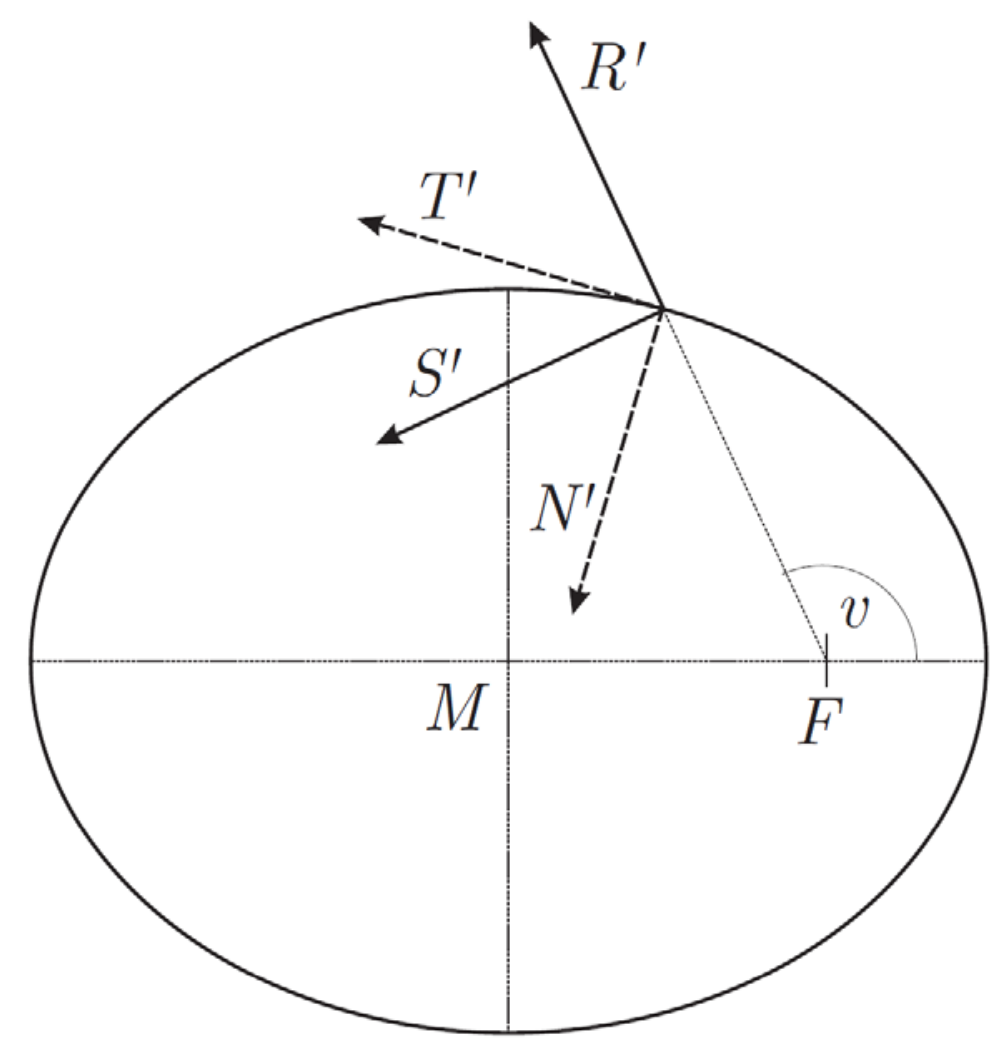

$\mathbf{R}, \mathbf{S}, \mathbf{C}$ unit vectors are pointing:

- into the radial direction

- normal to $\mathbf{R}$ in the orbital plane

- normal to the orbital plane (cross-track)

T, N, C unit vectors are pointing:

- into the tangential (along-track) direction

- normal to $\mathbf{T}$ in the orbital plane

- normal to the orbital plane (cross-track)

Small eccentricities: S T (velocity direction) 


\section{Orbit Differences KIN-RD (GOCE: begin of mission)}

Differences at epochs of kin. positions
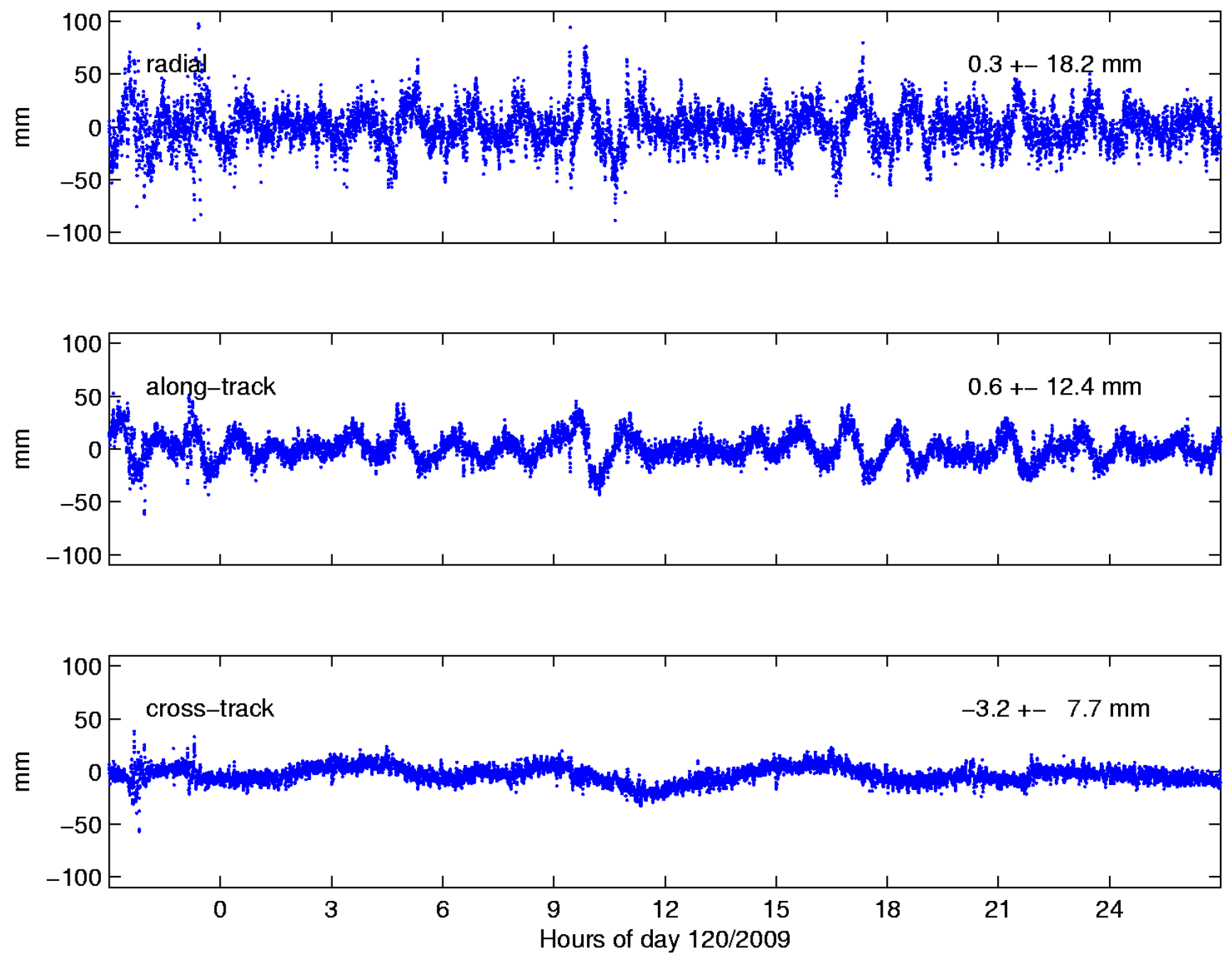


\section{Orbit Differences KIN-RD, Time-Differenced}

Largest scatter of kin. positions
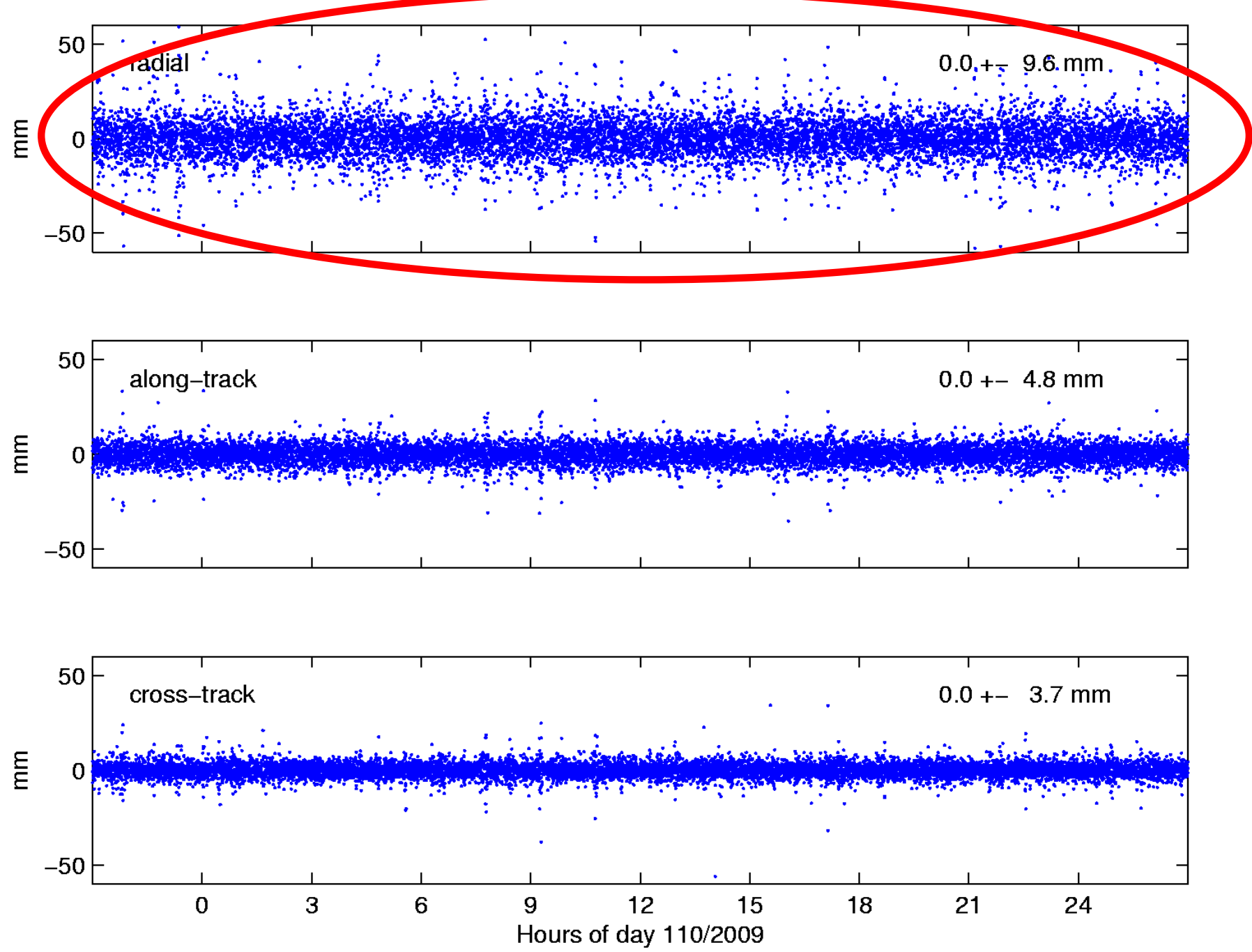


\section{Pseudo-Stochastic Accelerations (GOCE: Begin of Mission)}

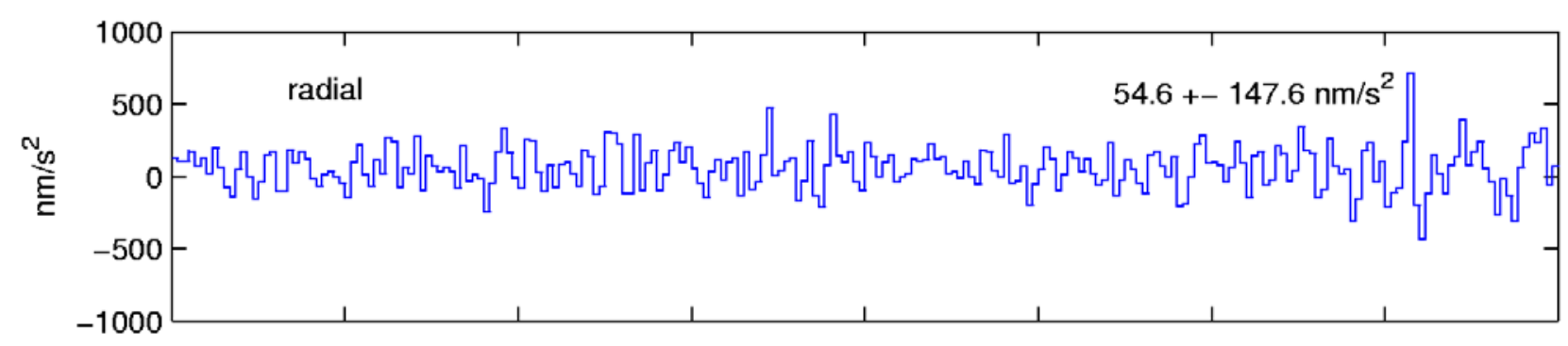

Earisgeblagigreed fligettøai7-diag
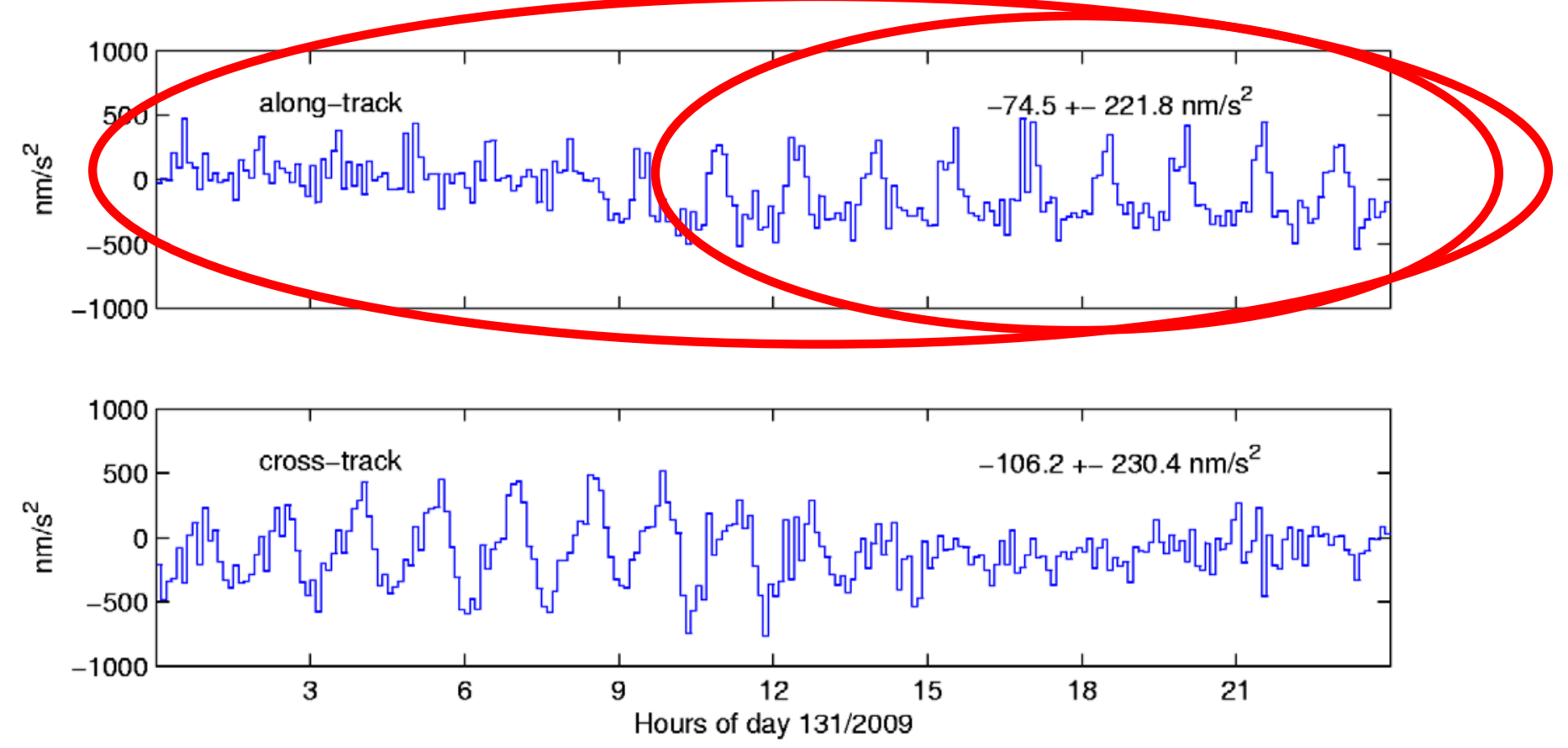

$A / U \boldsymbol{B}$ 


\section{Improving LEO Orbit Determination (1)}

PCV modeling is one of the limiting factors for most precise LEO orbit determination. Unmodeled PCVs are systematic errors, which

- directly propagate into kinematic orbit determination and severly degrade the position estimates

- propagate into reduced-dynamic orbit determination to a smaller, but still large extent

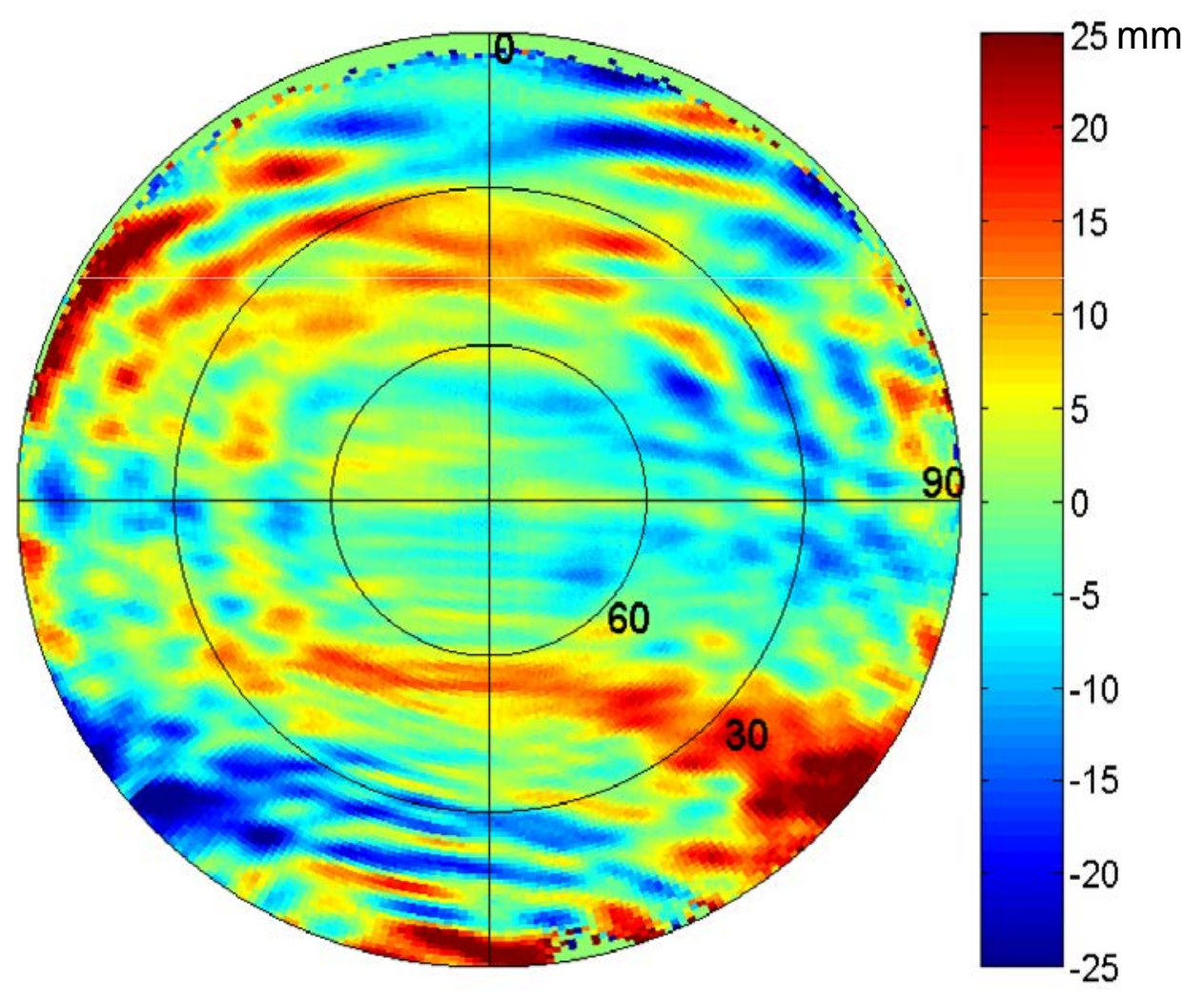




\section{Improving LEO Orbit Determination (2)}
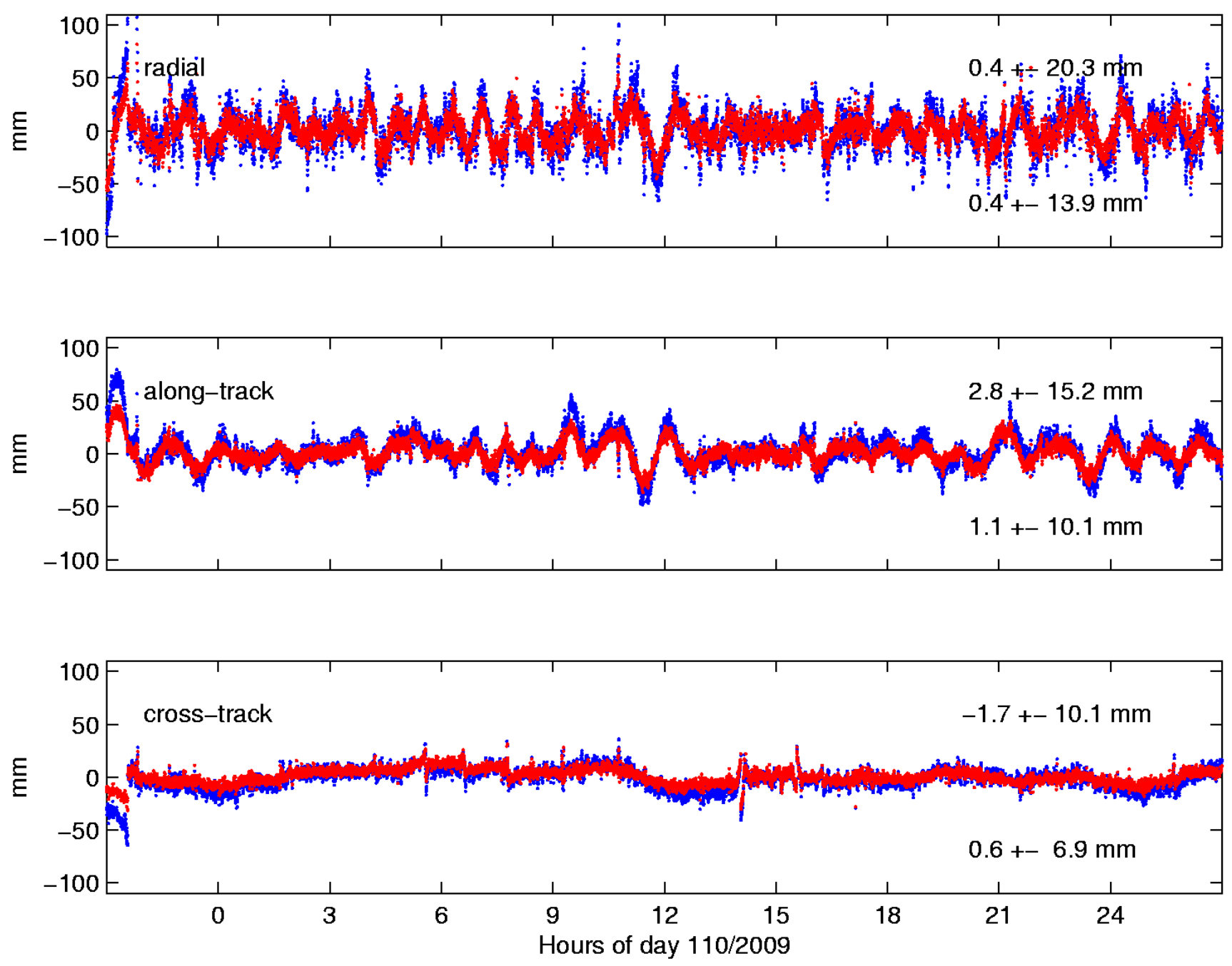


\section{Orbit Differences KIN-RD (GOCE: entire mission)}

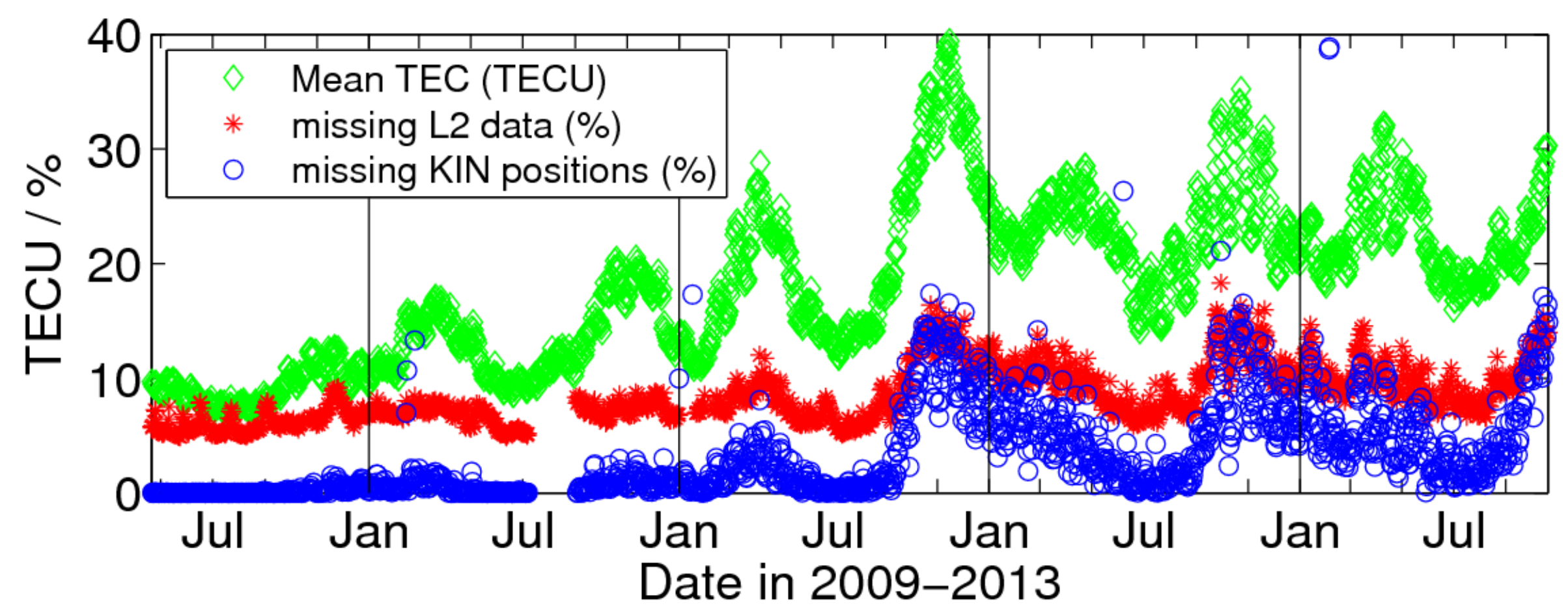

The result illustrates the consistency between both orbit-types. The level of the differences is usually given by the quality of the kinematic positions.

The differences are highly correlated with the ionosphere activity and with data losses on L2.

(Bock et al., 2014) 


\section{Orbit Differences KIN-RD (GOCE)}
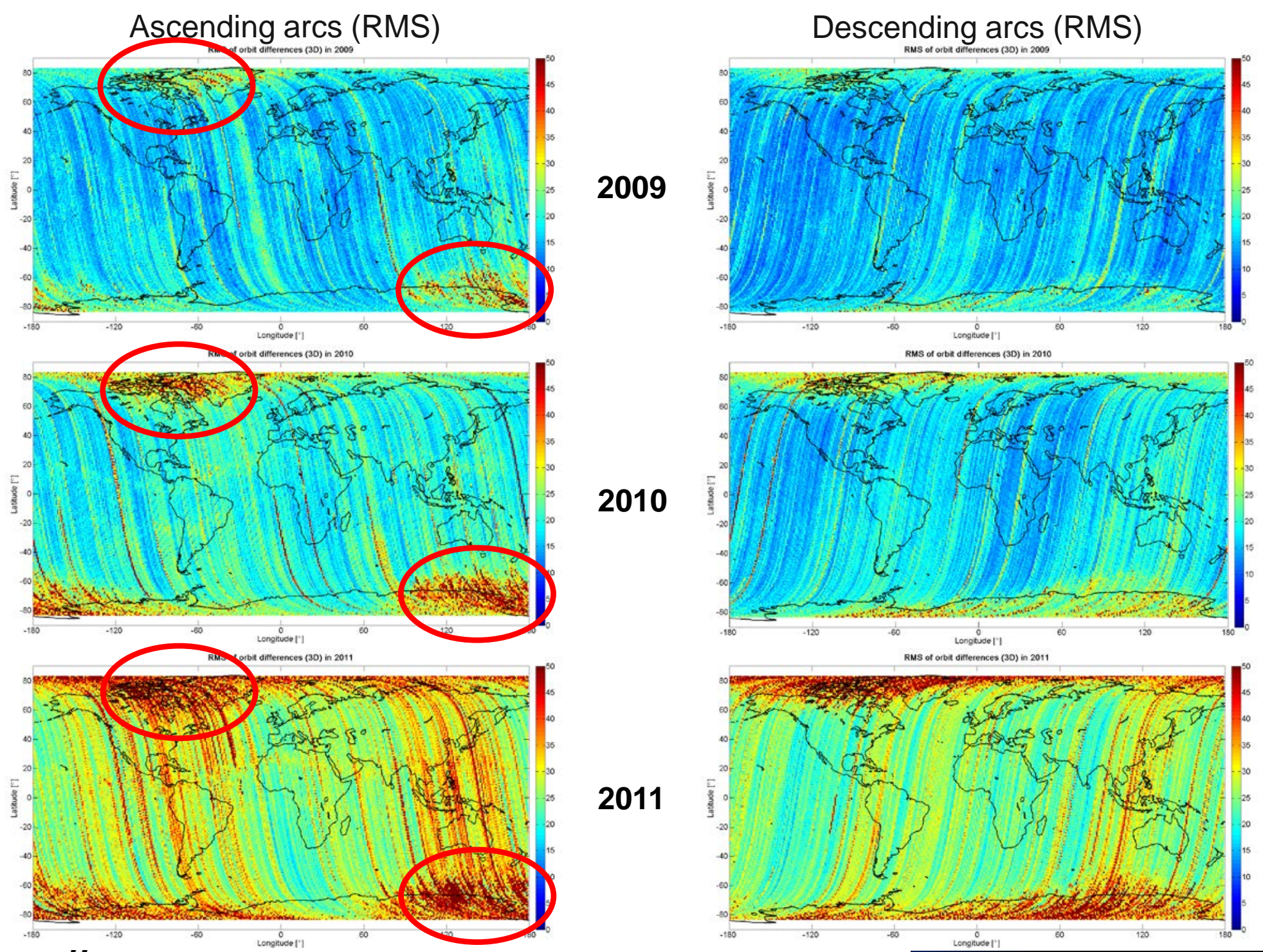

Bad Honnef, 04.10. - 09.10. 2015

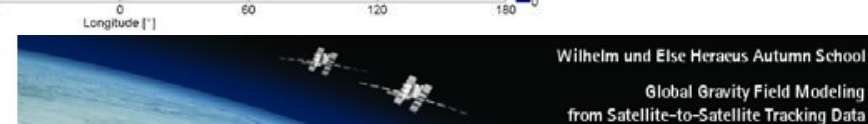




\section{Orbit Validation with SLR (GOCE)}

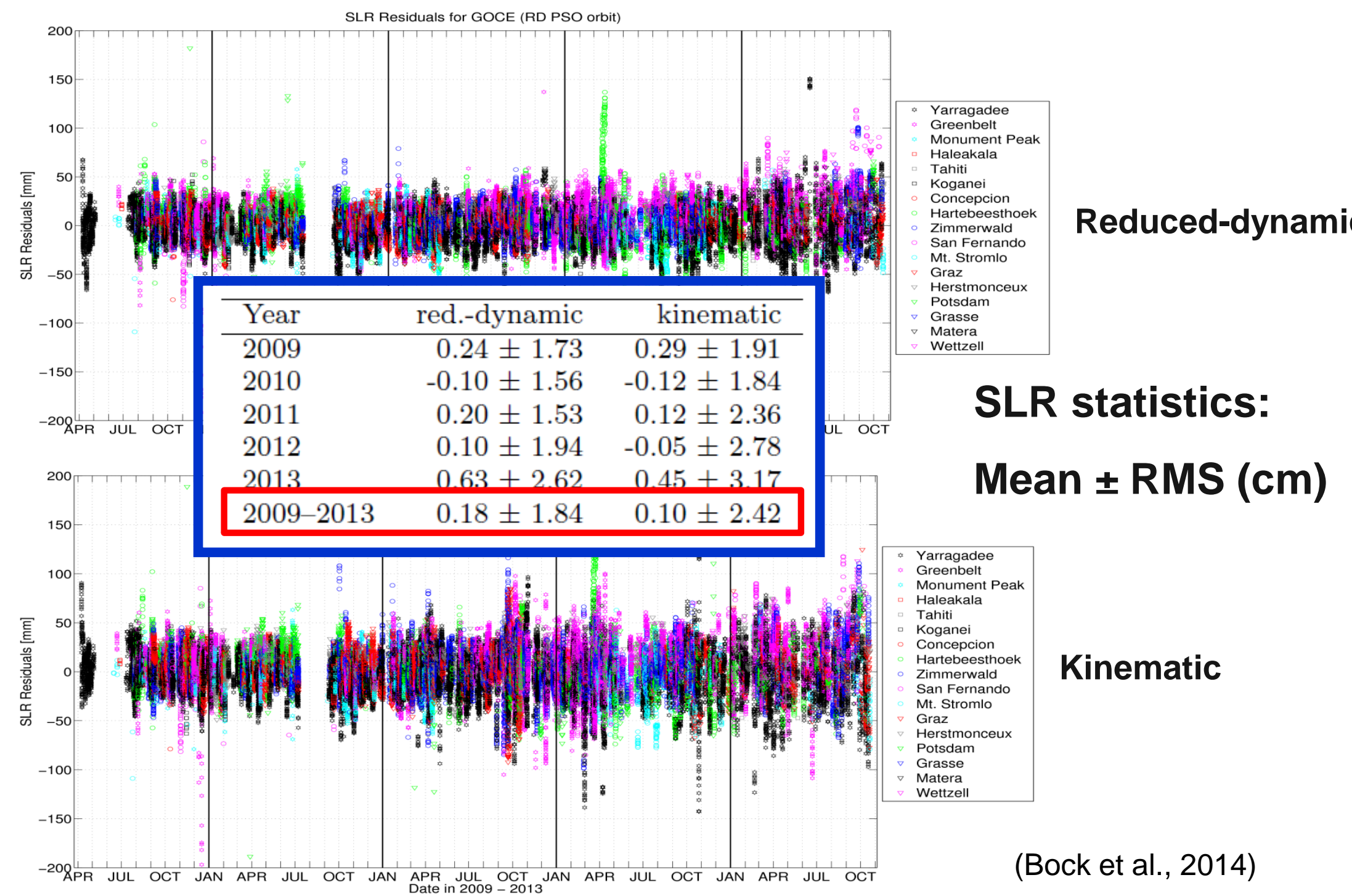




\section{Orbit Validation with SLR (GOCE)}

LEO orbits may be shifted up to several $\mathrm{cm}$ 's in the cross-track direction by unmodeled PCVs.

Thanks to the low orbital altitude of GOCE it could be confirmed for the first $E$ time with SLR data that the PCV-induced crosstrack shifts are real (see measurements from the SLR stations in the east and west directions at low elevations).

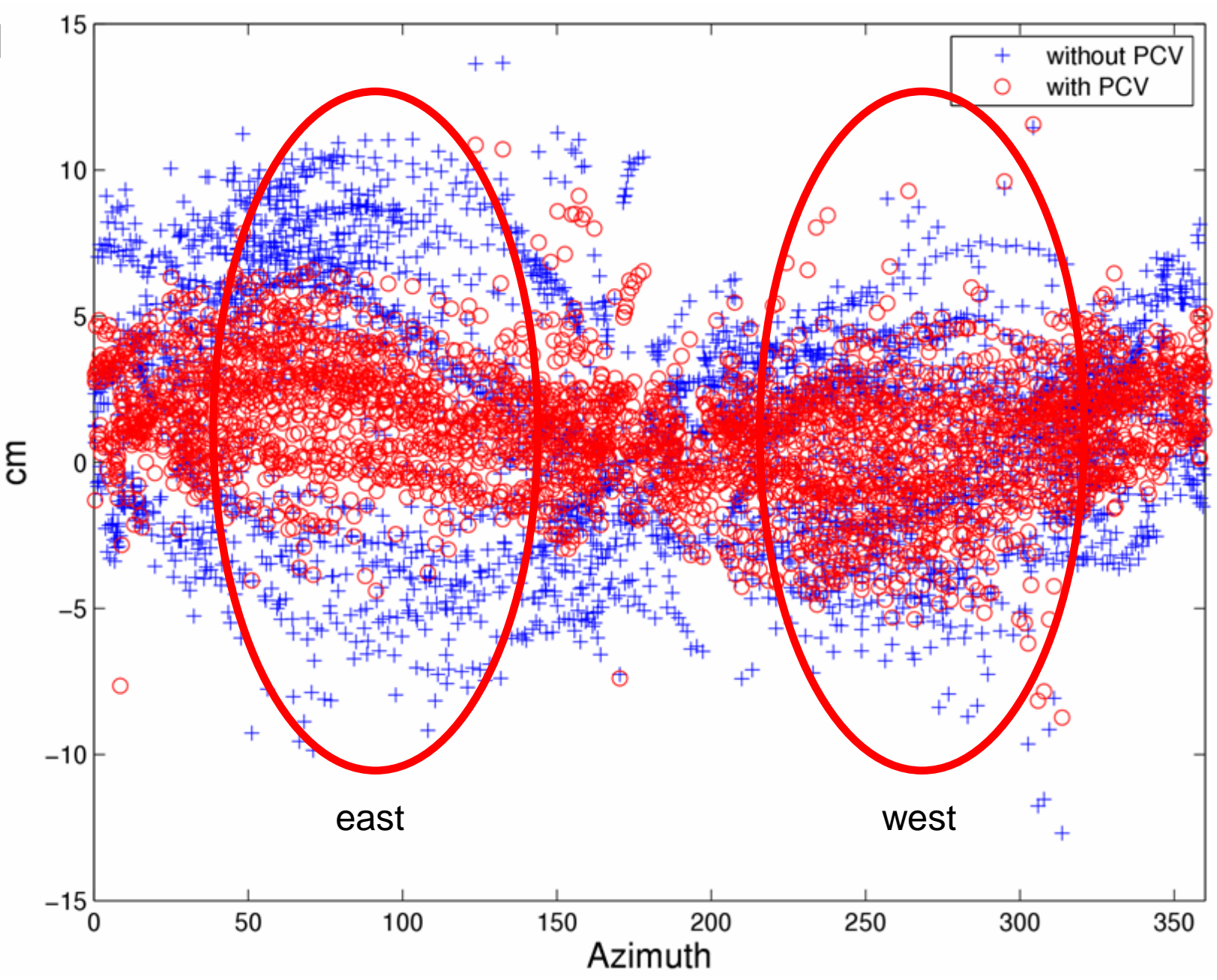




\section{SLR validation concepts}

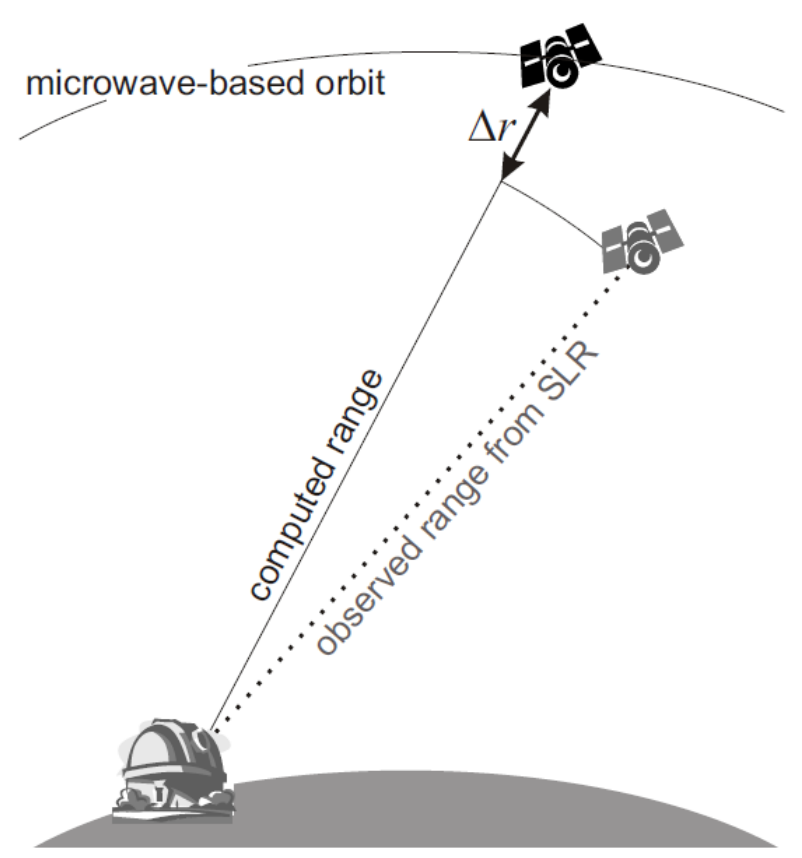

(Flohrer, 2008)

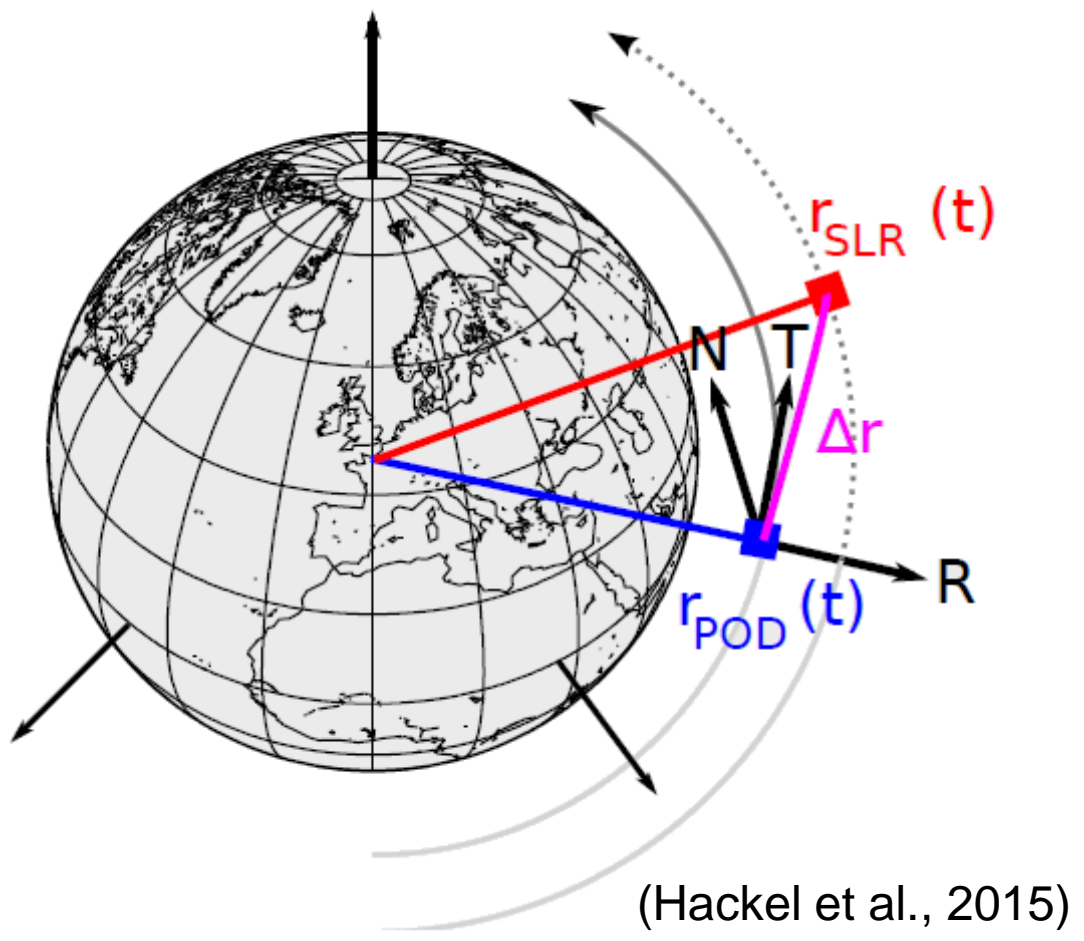

Especially LEO satellites at low orbital altitudes allow not only for a validation of the orbit quality in the radial direction, but also in the other directions. Using long data spans, mean SLR biases may be determined in along-track and cross-track, as well. 


\section{Other Validation Concepts (1)}

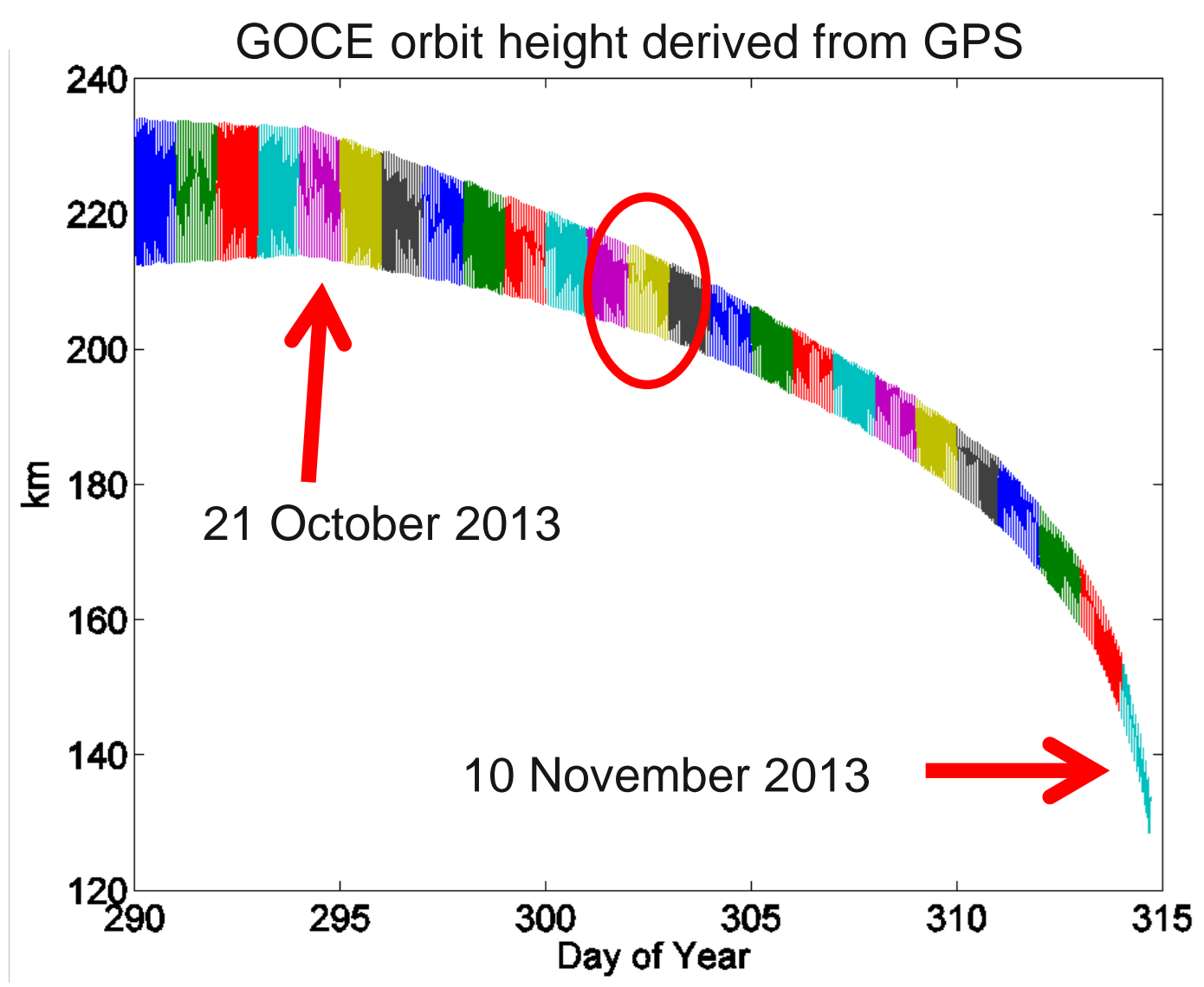

At extremely low orbital altitudes, the comparison between reduced-dynamic and kinematic orbits rather validates the quality of the reduced-dynamic orbits. 


\section{Other Validation Concepts (2)}
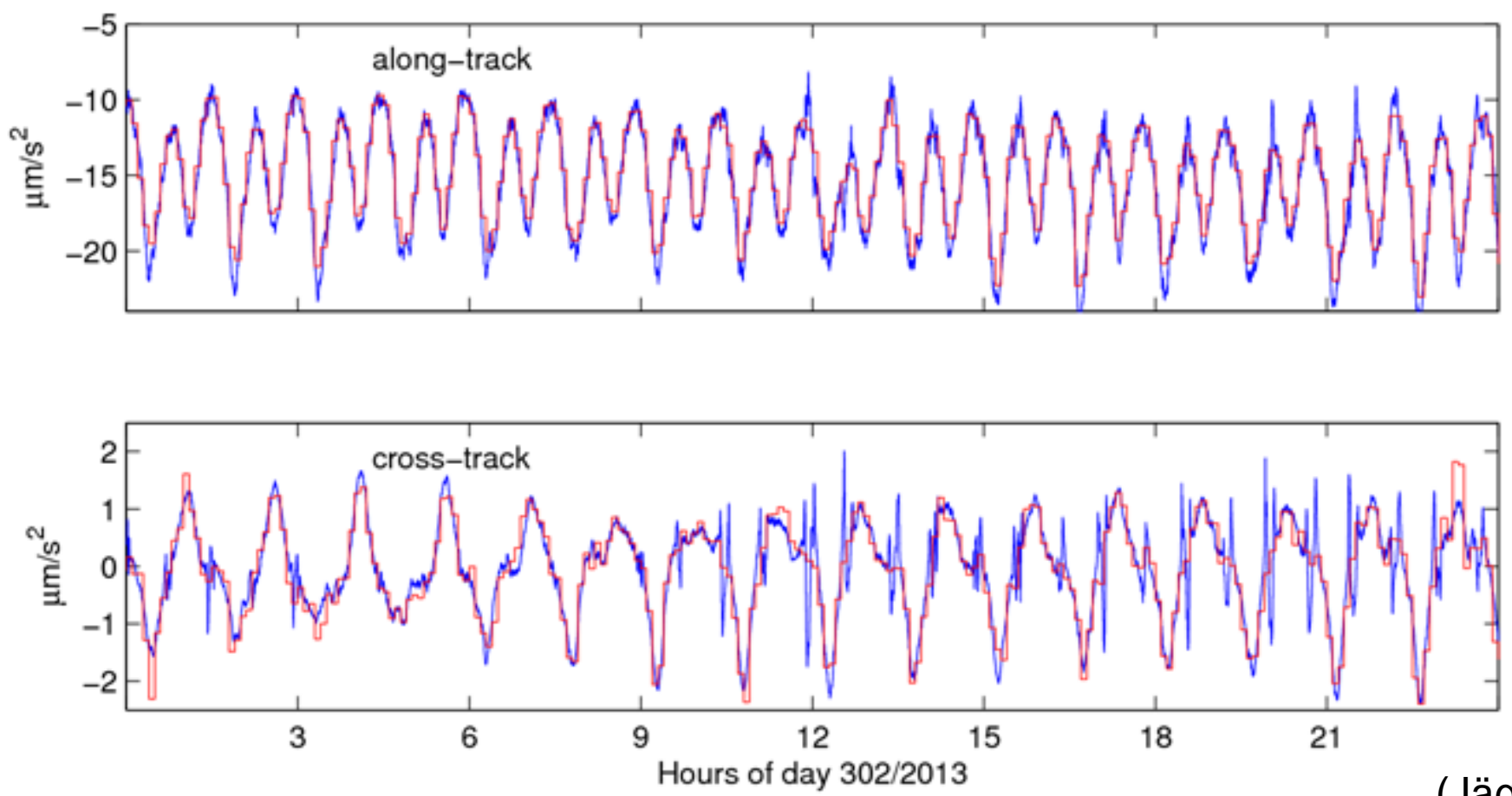

(Jäggi et al., 2014)

The comparison between estimated accelerations and measured data from onboard accelerometers may also provide an indication of the underlying orbit quality. If non-gravitational force models are taken into account, the magnitude of the estimated accelerations indicates the quality of the force modeling. 


\section{Non-gravitational Force Modeling (1)}

Swarm orbit solutions derived from GPS

\begin{tabular}{|c|c|c|c|c|c|}
\hline & & IDO & 107 & 1D8 & \\
\hline \multirow{2}{*}{ Ref. Frames } & IERS1996 & $\checkmark$ & & & [5] \\
\hline & IERS2010 & & $\checkmark$ & & [6] \\
\hline \multirow{2}{*}{ Gravitation } & GGM01S+TOP3.0 & $\checkmark$ & & & [7] \\
\hline & GOCO03S+FES4.0 & & $\checkmark$ & (a & [8] \\
\hline \multirow{3}{*}{ Radiation } & SRP cannon-ball & $\checkmark$ & & $\underline{\underline{\underline{y}}}$ & \\
\hline & SRP macro & & $\checkmark$ & $\geq$ & \\
\hline & ERP macro & & $\checkmark$ & $\frac{5}{0}$ & \\
\hline \multirow[b]{2}{*}{ Aerodyn. } & Drag cannon-ball & $\sqrt{ }$ & & $\stackrel{\cup}{\rightleftarrows}$ & \\
\hline & Drag macro & & & $\sum_{\varepsilon}^{0}$ & \\
\hline \multirow{3}{*}{ Atm. Density } & $\begin{array}{l}\text { Drag, lift, molec. } \\
\text { Jacchia-71G }\end{array}$ & $\checkmark$ & $\checkmark$ & $\frac{d}{\frac{1}{y}}$ & [3] \\
\hline & DTM-2012 & & & & [10] \\
\hline & NRLMSISE-00 & & $\checkmark$ & & [11] \\
\hline Wind & HWM-07 & & $\checkmark$ & & [12] \\
\hline
\end{tabular}

(Hackel et al., 2015) 


\section{Non-gravitational Force Modeling (2)}

Swarm orbit solutions derived from GPS

\begin{tabular}{cccccccc}
\hline ID & $C D$ & $C E$ & $C R$ & $a_{R}\left[\mathrm{~nm} / \mathrm{s}^{2}\right]$ & $\mathrm{a}_{T}\left[\mathrm{~nm} / \mathrm{s}^{2}\right]$ & $\mathrm{a}_{\mathrm{N}}\left[\mathrm{nm} / \mathrm{s}^{2}\right]$ & SLR Residuals $[\mathrm{mm}]$ \\
\hline id0 & $1.3 \pm 0.3$ & $\mathrm{n} / \mathrm{a} \pm \mathrm{n} / \mathrm{a}$ & $4.3 \pm 0.9$ & 9.4 & 36.9 & 24.3 & $-1.1 \pm 16.7$ \\
id7 & $0.8 \pm 0.2$ & $0.7 \pm 0.2$ & $1.0 \pm 0.2$ & 1.5 & 22.2 & 14.0 & $0.7 \pm 15.9$ \\
id8 & $\mathrm{n} / \mathrm{a} \pm \mathrm{n} / \mathrm{a}$ & $\mathrm{n} / \mathrm{a} \pm \mathrm{n} / \mathrm{a}$ & $\mathrm{n} / \mathrm{a} \pm \mathrm{n} / \mathrm{a}$ & $\mathrm{n} / \mathrm{a}$ & $\mathrm{n} / \mathrm{a}$ & $\mathrm{n} / \mathrm{a}$ & $-0.7 \pm 38.2$ \\
\hline
\end{tabular}

Quite an effort is needed to actually improve the quality of reduced-dynamic trajectories by taking into account non-gravitational force models. If dense and continuous tracking data (such as GPS) are available, the pseudo-stochastic orbit modeling techniques are very powerful.

But of course, a good modeling is always preferred. This guarantees that the trajectories have a good dynamical stiffness and are less prone to data problems and outages. But for results of highest quality, empirical parameters are usually nevertheless needed ... 


\section{Purely Dynamic LEO Orbit Modeling}

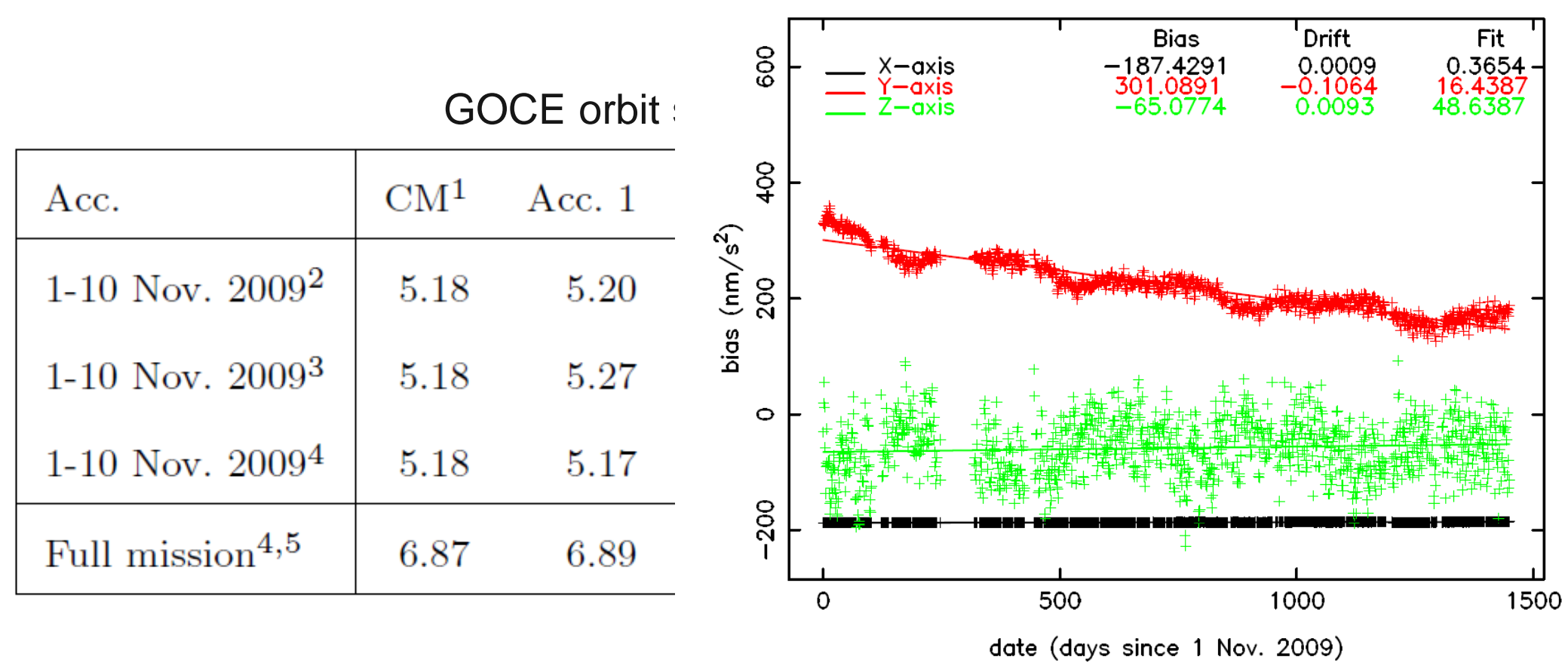

Thanks to the outstanding quality of the GOCE accelerometers, purely dynamic orbit determination is feasible. The table shows that the agreement with the official Precise Science Orbits is about $5 \mathrm{~cm}$ when using the common-mode or even the individual accelerometer data. Accelerometer calibration parameters are estimated together with the initial conditions as the only additional parameters.

(Visser et al., 2015) 


\section{GRACE Orbit Validation with K-Band (1)}

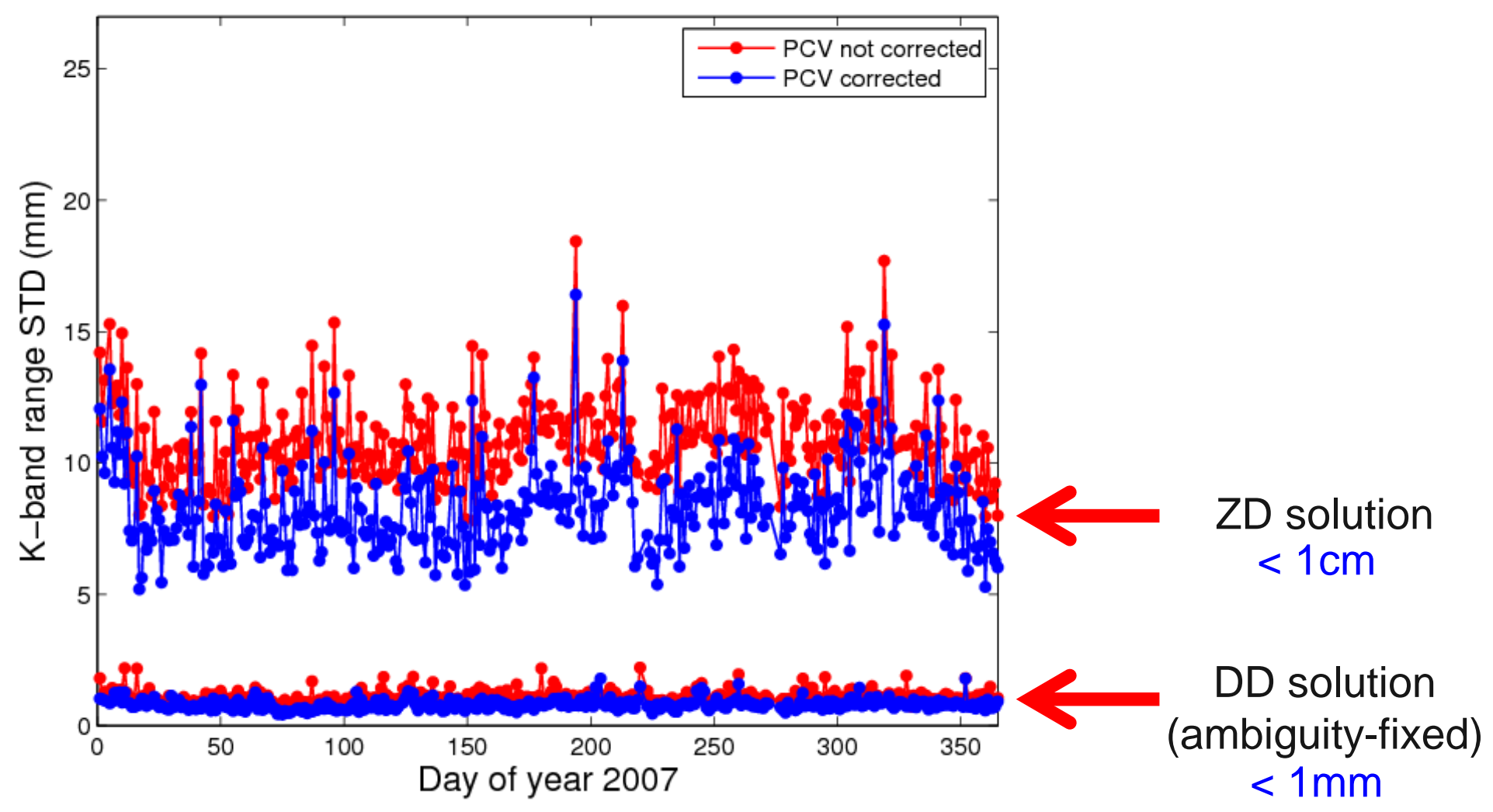

The ultra-precise and continuously available K-Band data allow it to validate the inter-satellite distances between the GRACE satellites. Thanks to this validation, e.g., PCV maps were recognized to be crucial for high-quality POD.

(Jäggi et al., 2009) 


\section{GRACE Orbit Validation with K-Band (2)}
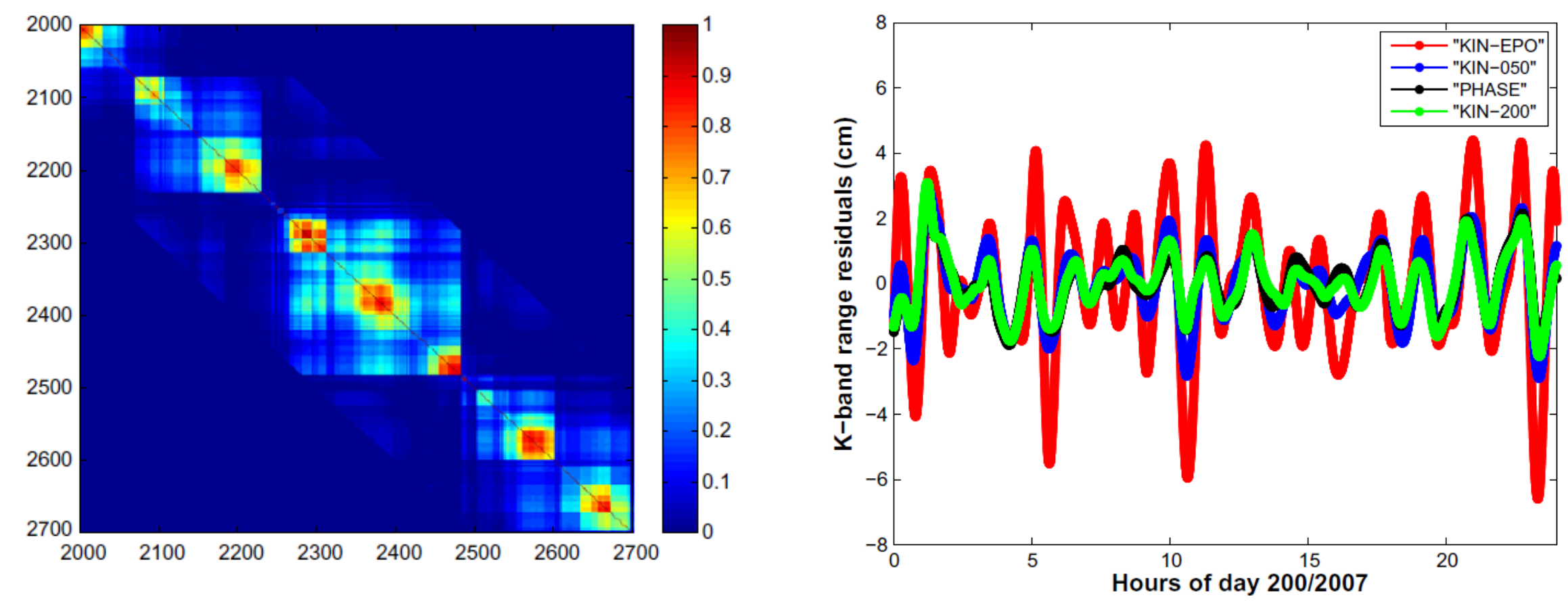

Reduced-dynamic fits through kinematic positions only then have the same quality as reduced-dynamic orbits directly derived from GPS carrier phase, if covariance information from the kinematic positioning is used over sufficiently long intervals to properly weight the kinematic pseudo-observations in the orbit determination.

(Jäggi et al., 2011b) 


\section{Generalized Orbit Determination}




\section{Generalized Orbit Determination (1)}

The actual orbit $\boldsymbol{r}(t)$ is expressed as a truncated Taylor series:

$$
\mathbf{r}(t)=\mathbf{r}_{0}(t)+\sum_{i=1}^{n_{O}} \frac{\partial \mathbf{r}_{0}(t)}{\partial O_{i}} \cdot o_{i}+\sum_{i=1}^{d} \frac{\partial \mathbf{r}_{0}(t)}{\partial Q_{i}} \cdot q_{i}
$$

$\mathbf{r}_{0}(t)$

$\frac{\partial \mathbf{r}_{0}(t)}{\partial O_{i}}, \frac{\partial \mathbf{r}_{0}(t)}{\partial Q_{i}}$

$o_{i}, q_{i}$
A priori orbit

Partials w.r.t. arc-specific and dynamic (global) parameters Corrections of arc-specific and dynamic (global) parameters

The variational equations of the dynamic parameters, e.g., gravity field coefficients, may be solved by the general methods as discussed earlier in this lecture. Their solutions may be reduced to definite integrals and efficiently solved by numerical quadrature. 


\section{Generalized Orbit Determination (2)}

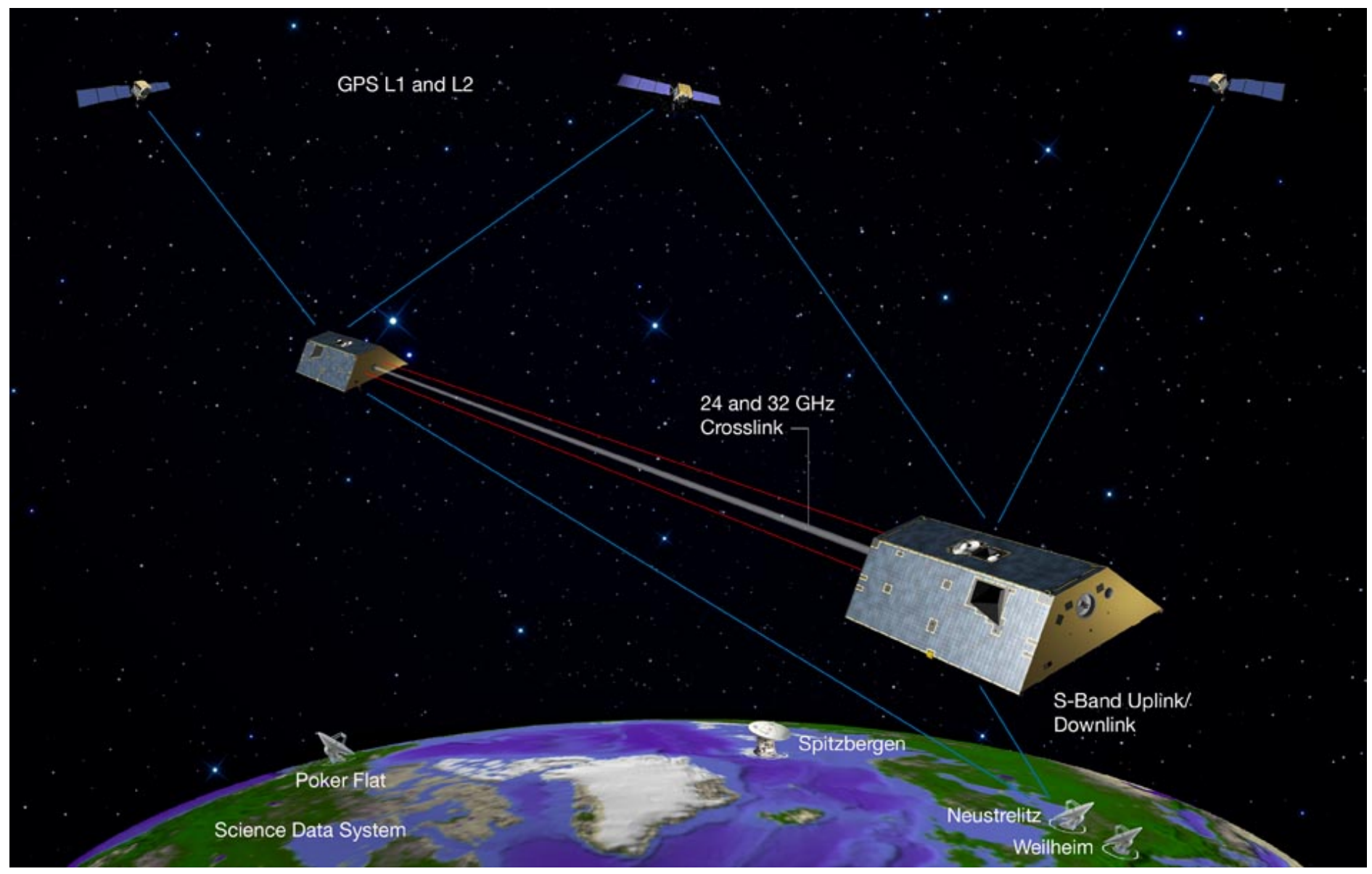




\section{Generalized Orbit Determination (3)}

The actual orbit difference $\mathbf{r}_{a}(t)-\mathbf{r}_{b}(t)$ is expressed as:

$$
\begin{aligned}
\mathbf{r}_{a}(t)-\mathbf{r}_{b}(t)= & \mathbf{r}_{a 0}(t)-\mathbf{r}_{b 0}(t) \\
& +\sum_{i=1}^{n_{a} O} \frac{\partial \mathbf{r}_{a 0}(t)}{\partial O_{a i}} \cdot o_{a i} \\
& -\sum_{i=1}^{n_{b O}} \frac{\partial \mathbf{r}_{b 0}(t)}{\partial O_{b i}} \cdot o_{b i} \\
& +\sum_{i=1}^{d}\left(\frac{\partial \mathbf{r}_{a 0}(t)}{\partial Q_{i}}-\frac{\partial \mathbf{r}_{b 0}(t)}{\partial Q_{i}}\right) \cdot q_{i}
\end{aligned}
$$

In order to set-up the observation equations, the partial derivatives of the a priori orbits need to be related to the observables, e.g., by projecting the respective terms on the line-of-sight direction between GRACE-A and -B in the case of K-Band (biased) range observations. 


\section{Generalized Orbit Determination (4)}

In case of GPS-based gravity field determination, the observation equations contain corrections for arc-specific parameters $\mathbf{o}$ and for (global) dynamic parameters q

$$
\varepsilon=\mathbf{A}_{o} \mathbf{o}+\mathbf{A}_{q} \mathbf{q}-\mathbf{l}
$$

The corresponding normal equation system reads as

$$
\left(\begin{array}{ll}
\mathbf{N}_{o o} & \mathbf{N}_{o q} \\
\mathbf{N}_{o q}^{T} & \mathbf{N}_{q q}
\end{array}\right) \cdot\left(\begin{array}{l}
\mathbf{o} \\
\mathbf{q}
\end{array}\right)=\left(\begin{array}{l}
\mathbf{b}_{o} \\
\mathbf{b}_{q}
\end{array}\right)
$$

and, after pre-elimination of the arc-specific parameters, as

$$
\left(\mathbf{N}_{q q}-\mathbf{N}_{o q}^{T} \mathbf{N}_{o o}^{-1} \mathbf{N}_{o q}\right) \mathbf{q}=\mathbf{b}_{q}-\mathbf{N}_{o q}^{T}\left(\mathbf{N}_{o o}^{-1} \mathbf{b}_{o}\right)
$$




\section{Orbit Fixation}

For didactic reasons, let us now fix the arc-specific parameters to previously determined values while estimating the corrections to the gravity field parameters in a second step. This implies that the sub-system

$$
\mathbf{N}_{o o} \mathbf{o}^{\prime}=\mathbf{b}_{o}
$$

is solved independently from the remaining part of the correct normal equation system and that the parameters $\mathbf{o}^{\prime}$ are introduced in the following gravity recovery step as known. The remaining normal equation system reads as

$$
\mathbf{N}_{q q} \mathbf{q}^{\prime}=\mathbf{b}_{q}-\mathbf{N}_{o q}^{T} \mathbf{o}^{\prime}=\mathbf{b}_{g}-\mathbf{N}_{o q}^{T}\left(\mathbf{N}_{o o}^{-1} \mathbf{b}_{o}\right)
$$

This yields a different (biased) solution as the orbit parameters $\mathbf{o}^{\prime}$ fully depend on the a priori gravity model and the correlations between orbit and gravity field parameters are ignored.

(Meyer et al., 2015) 


\section{Relation to the Acceleration Approach (1)}

Let us assume that the second derivatives of the position vector have been observed (derived by numerical differentiation from kinematic positions). The observation equations for one particular epoch read as

$$
\varepsilon_{r}=\sum_{k=1}^{n_{0}} \frac{\partial \ddot{\mathbf{r}}\left(t_{r}\right)}{\partial O_{k}} \cdot o_{k}+\sum_{k=1}^{d} \frac{\partial \ddot{\mathbf{r}}\left(t_{r}\right)}{\partial Q_{k}} \cdot q_{k}-\Delta \ddot{\mathbf{r}}_{r}
$$

where $\Delta \ddot{\mathbf{r}}_{r}$ represents the observed minus the computed acceleration. The partial derivatives in the second sum may be replaced by the right-hand sides of the variational equations, which read as

$$
\frac{\partial \ddot{\mathbf{r}}\left(t_{r}\right)}{\partial Q_{k}}=\frac{\partial \mathbf{f}\left(t_{r}\right)}{\partial \mathbf{r}\left(t_{r}\right)} \cdot \frac{\partial \mathbf{r}\left(t_{r}\right)}{\partial Q_{k}}+\frac{\partial \mathbf{f}\left(t_{r}\right)}{\partial \dot{\mathbf{r}}\left(t_{r}\right)} \cdot \frac{\partial \dot{\mathbf{r}}\left(t_{r}\right)}{\partial Q_{k}}+\frac{\partial \mathbf{f}\left(t_{r}\right)}{\partial Q_{k}}
$$




\section{Relation to the Acceleration Approach (2)}

The observation equations actually used in the acceleration approach read as

$$
\varepsilon_{r}=\sum_{k=1}^{d} \frac{\partial \mathbf{f}\left(t_{r}\right)}{\partial Q_{k}} \cdot q_{k}-\Delta \ddot{\mathbf{r}}_{r}
$$

From the point of view of orbit determination this implies that

$$
\sum_{k=1}^{n_{0}} \frac{\partial \ddot{\mathbf{r}}\left(t_{r}\right)}{\partial O_{k}} \cdot o_{k}+\sum_{k=1}^{d}\left(\frac{\partial \mathbf{f}\left(t_{r}\right)}{\partial \mathbf{r}\left(t_{r}\right)} \cdot \frac{\partial \mathbf{r}\left(t_{r}\right)}{\partial Q_{k}}+\frac{\partial \mathbf{f}\left(t_{r}\right)}{\partial \dot{\mathbf{r}}\left(t_{r}\right)} \cdot \frac{\partial \dot{\mathbf{r}}\left(t_{r}\right)}{\partial Q_{k}}\right)=\mathbf{0}
$$

It is thus assumed that the changes in the second derivatives of the orbit caused by the estimated gravity field parameters are counterbalanced by changes of the second derivatives of the orbit due to the changes in the arc-specific parameters. The assumption is met if the a priori orbit used to compute $\Delta \ddot{\mathbf{r}}_{r}$ in the acceleration approach equals the estimated a posteriori orbit from classical orbit determination. 


\section{Gravity Fiel Solutions from Kinematic Positions}

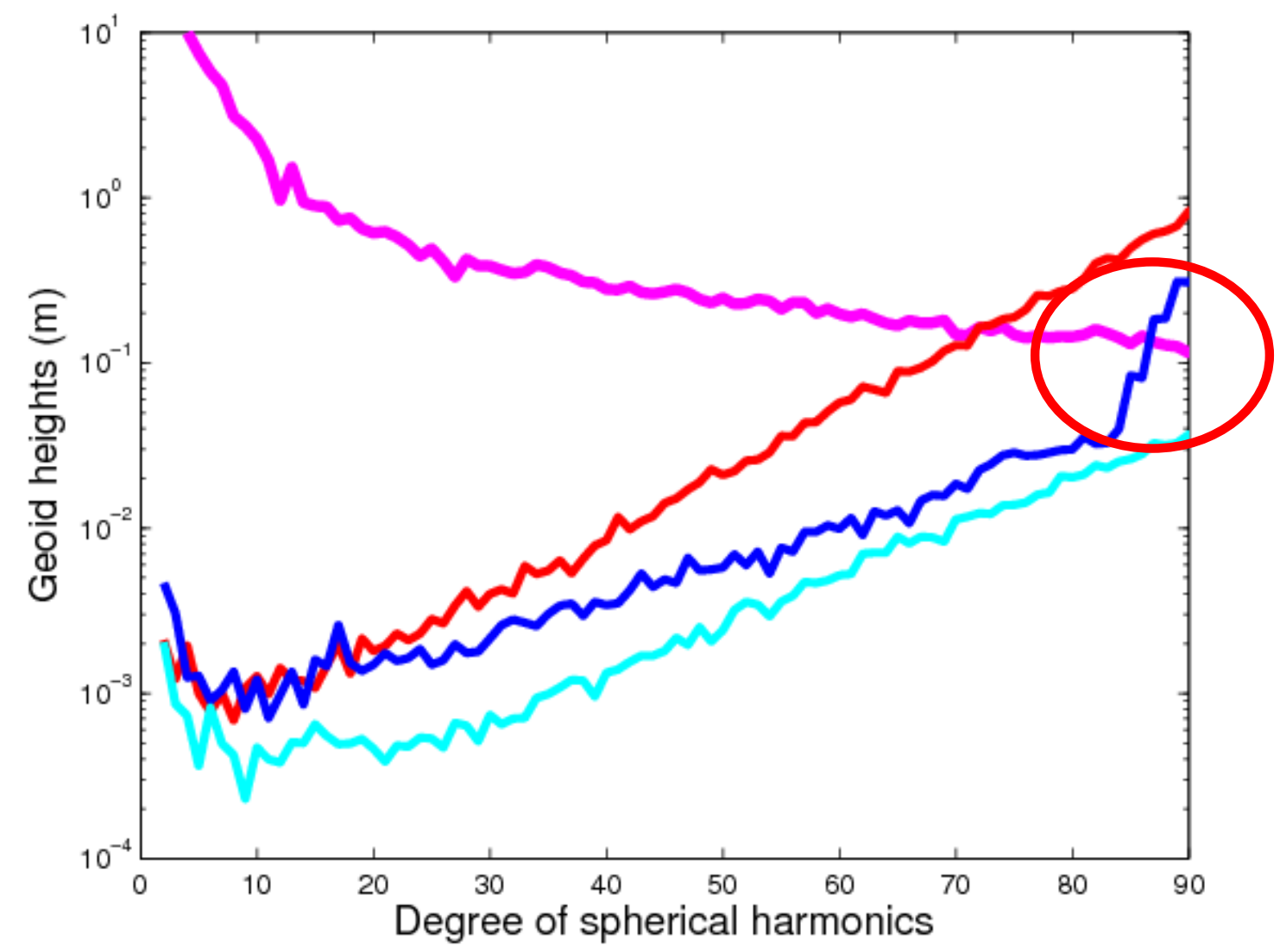

Reference field:

GRACE Solution

Differences:

1-year GRACE

0.7-years GOCE

8-years CHAMP

Different slopes of the difference degree amplitudes due to different LEO altitudes.

(Jäggi et al., 2011a)

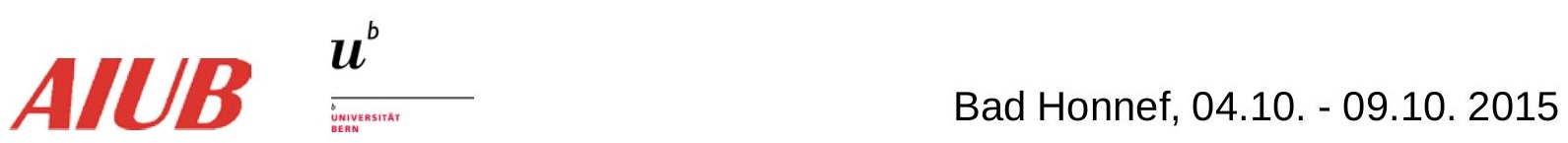




\section{Impact of PCVs on GPS-Based Gravity Field Recovery}

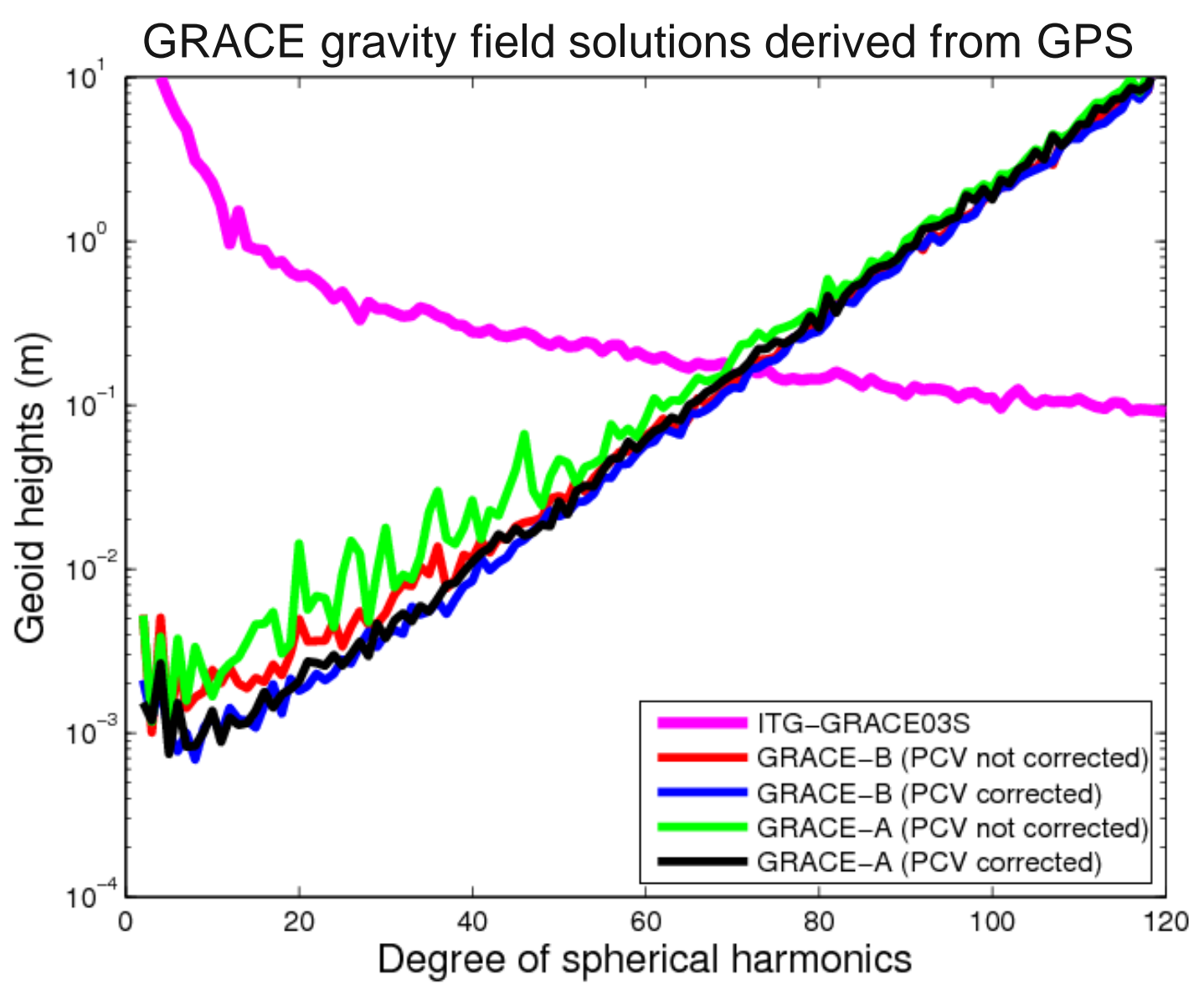

Mismodeled PCV maps may propagate via kinematic positions into the gravity field solutions. They represent a significant source for systematic POD errors.

(Jäggi et al., 2009) 


\section{Impact of PCVs on GPS-Based Gravity Field Recovery}

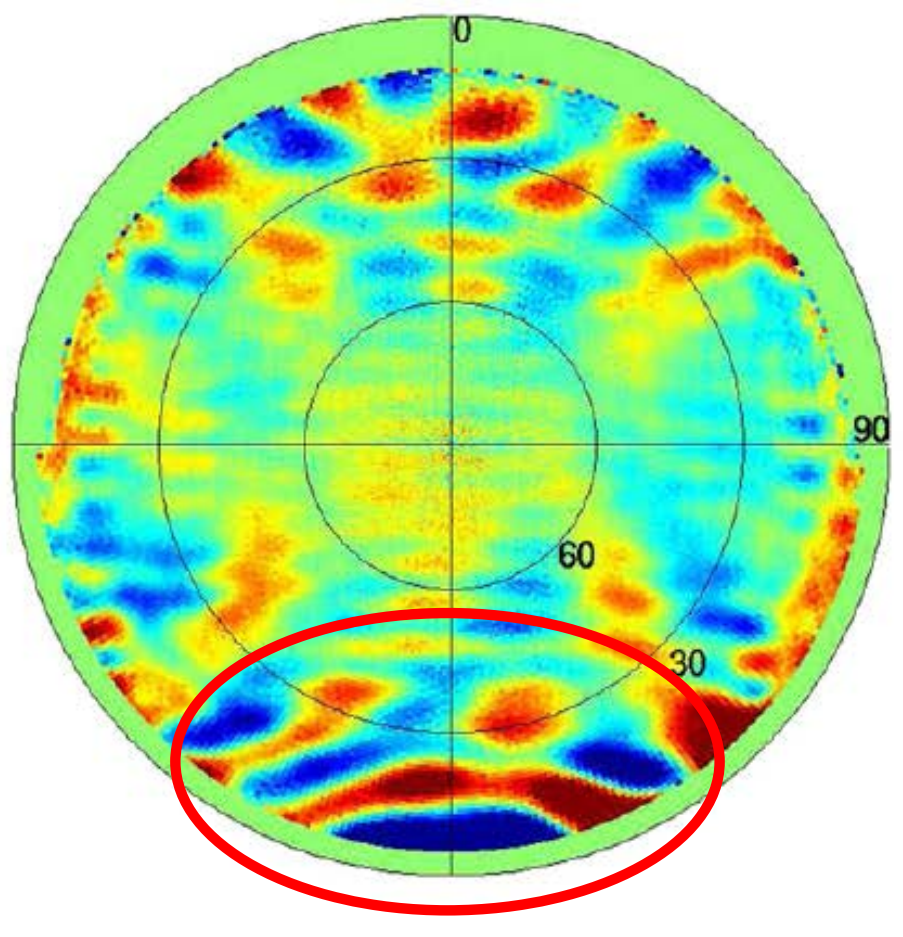

GRACE-A

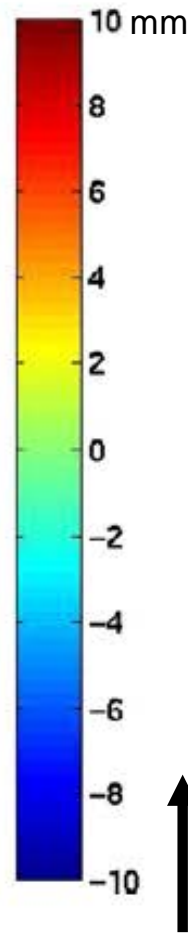

flight

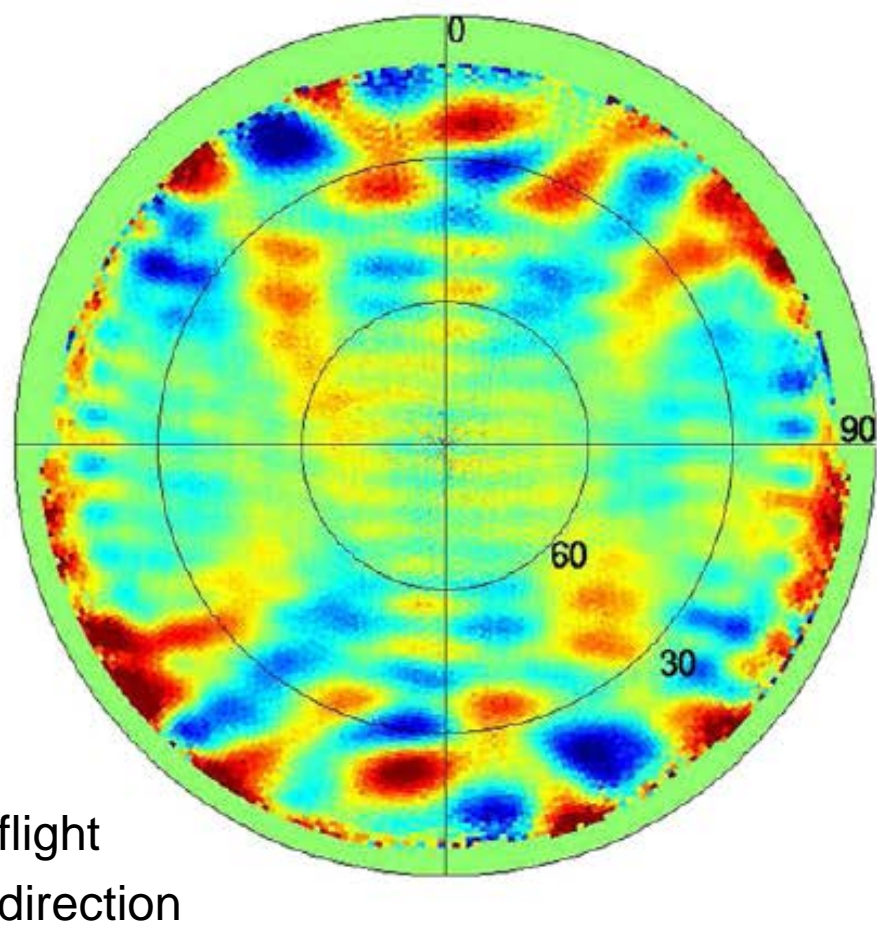

GRACE-B

(occultation antenna switched on)

(Jäggi et al., 2009) 


\section{Other Sources of Systematic Errors}

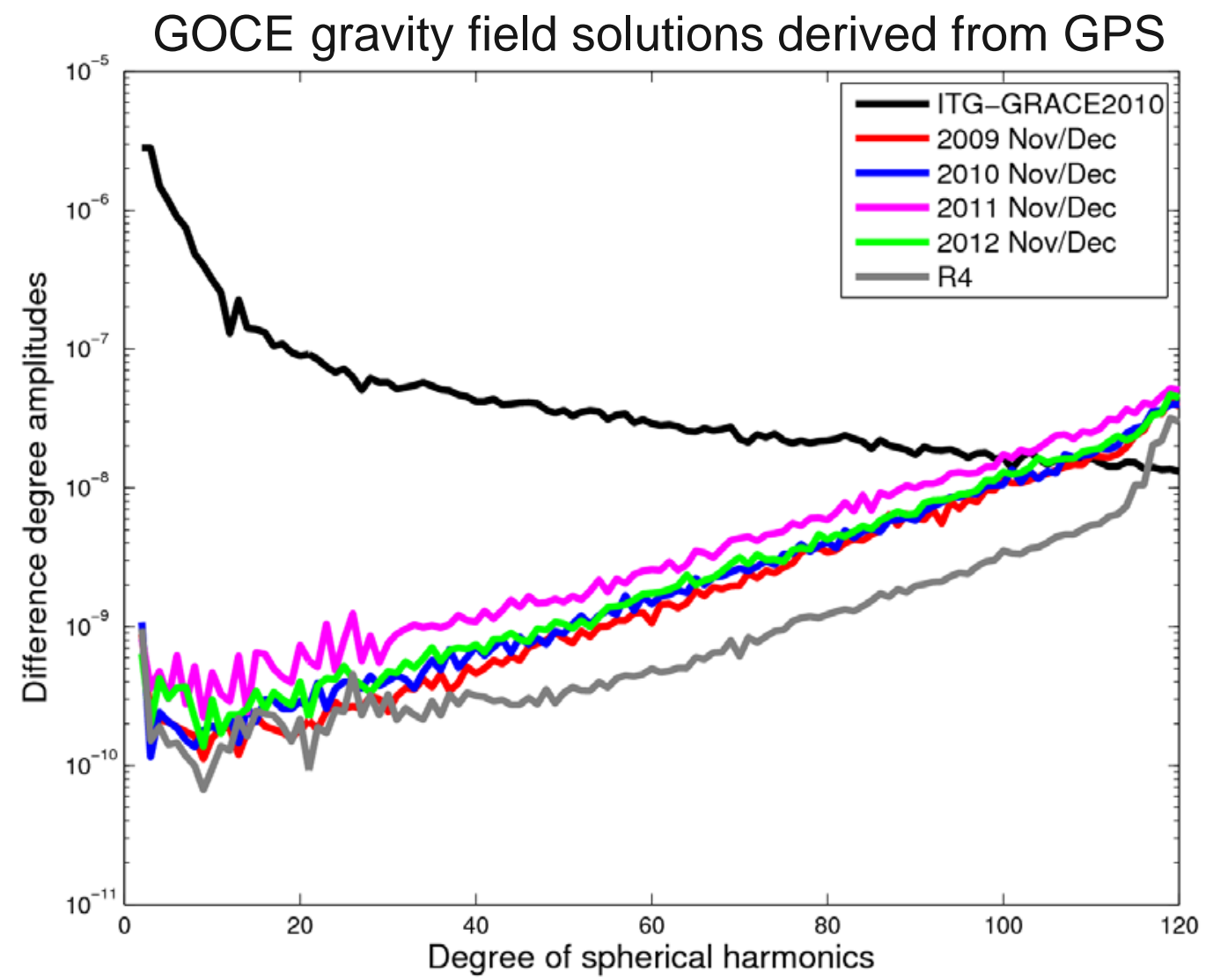

Significantly different qualities of various bi-monthly GOCE GPS-only solutions. The long-term solution R4 shows no significantly improved quality w.r.t. the bimonthly solutions below degree 30 .

(Jäggi et al., 2015) 


\section{Ionospheric Effects in the Orbits (1)}

Systematic effects around the geomagnetic equator are present in the ionosphere-free GPS phase residuals => affects kinematic positions

Degradation of kinematic positions around the geomagnetic equator propagates into gravity field solutions.

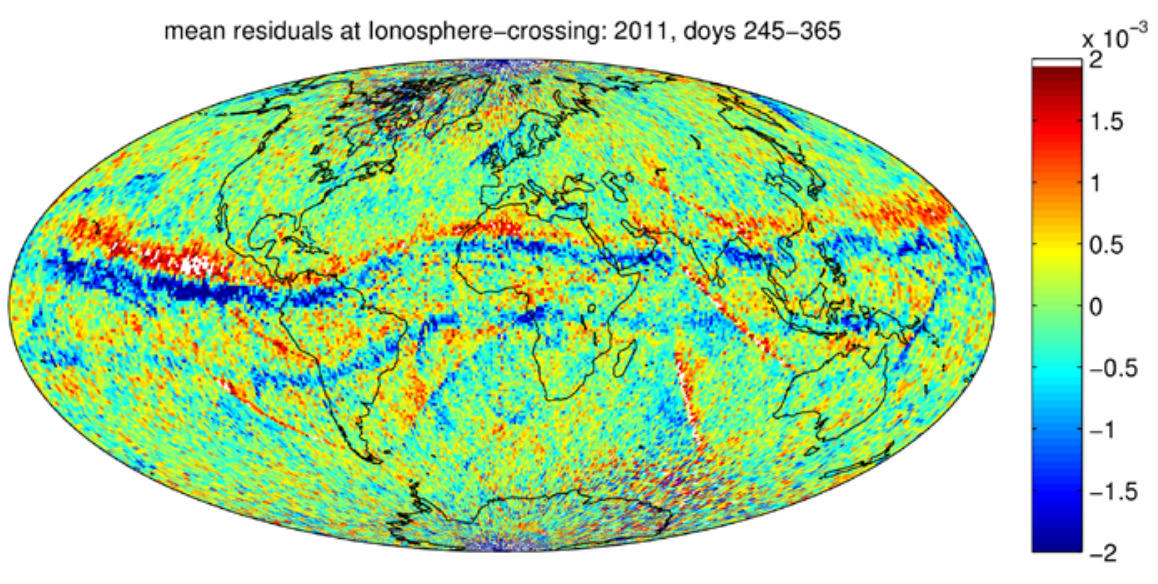

Phase observation residuals

$(-2 \mathrm{~mm} \ldots+2 \mathrm{~mm})$ mapped to the ionosphere piercing point

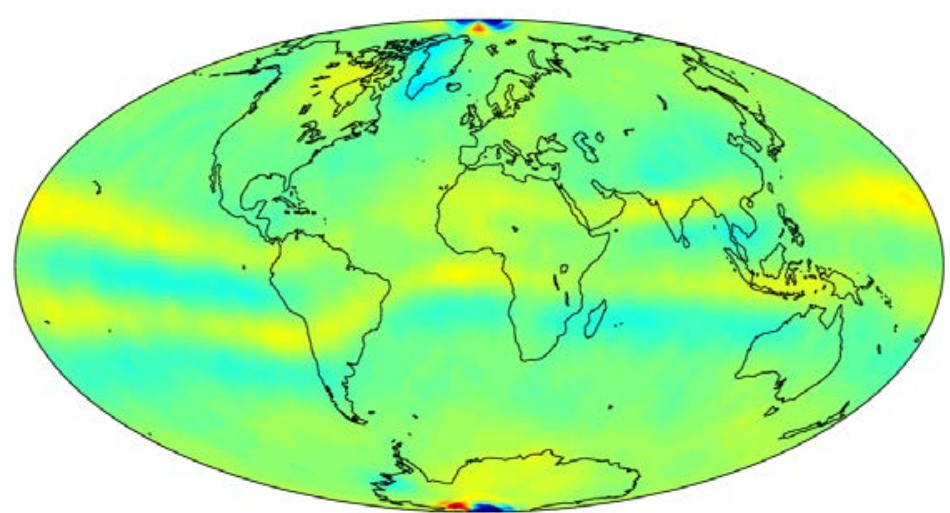

Geoid height differences

$(-5 \mathrm{~cm} \mathrm{\ldots .} 5 \mathrm{~cm})$;

TIM-R4 model

(Jäggi et al., 2015a) 


\section{Ionospheric Effects in the Orbits (2)}

One possible cause is the neglection of the higher order ionosphere $(\mathrm{HOI})$ correction terms.

First tests using $\mathrm{HOI}$ correction terms did, however, not show any improvement in the results.

But an empirical approach can be adopted:

Removal of observations, which have large ionosphere changes from one epoch to the next (e.g. $>5 \mathrm{~cm} / \mathrm{s})$.
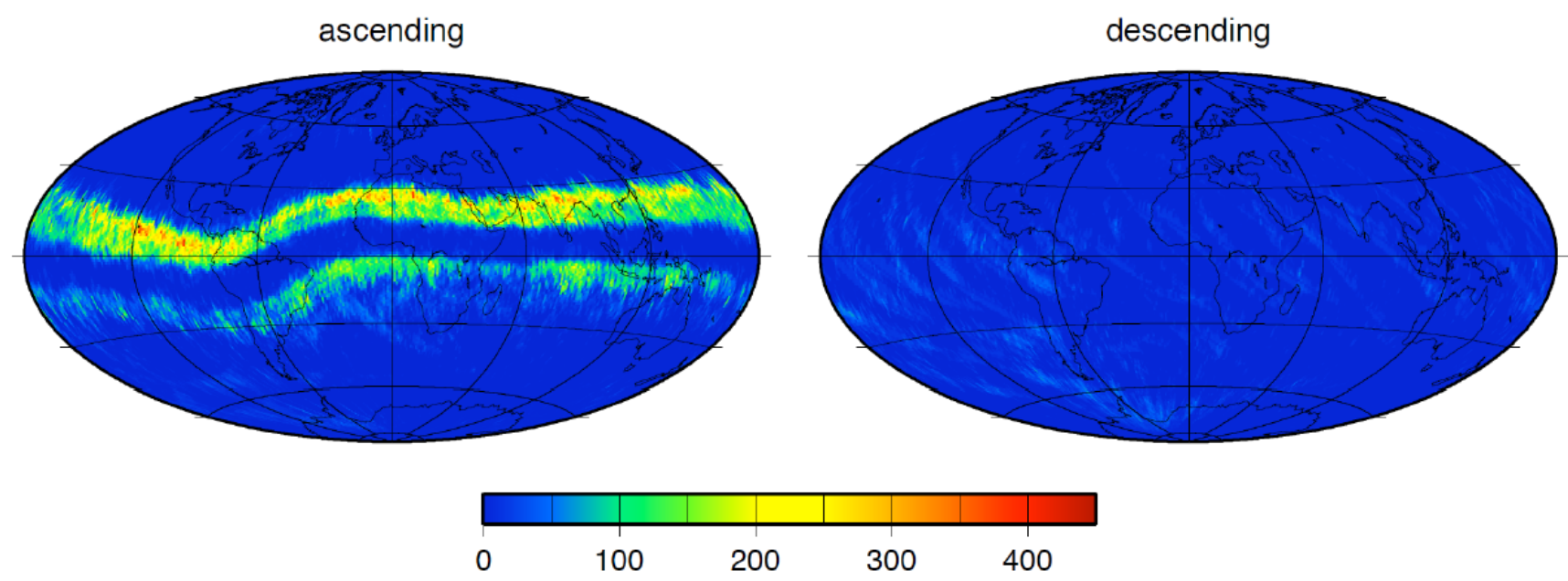

$\mathrm{nr}$ of removed points in $1 \times 1$ bins for doy 300-365 in 2011 


\section{Ionospheric Effects in the Orbits (3)}

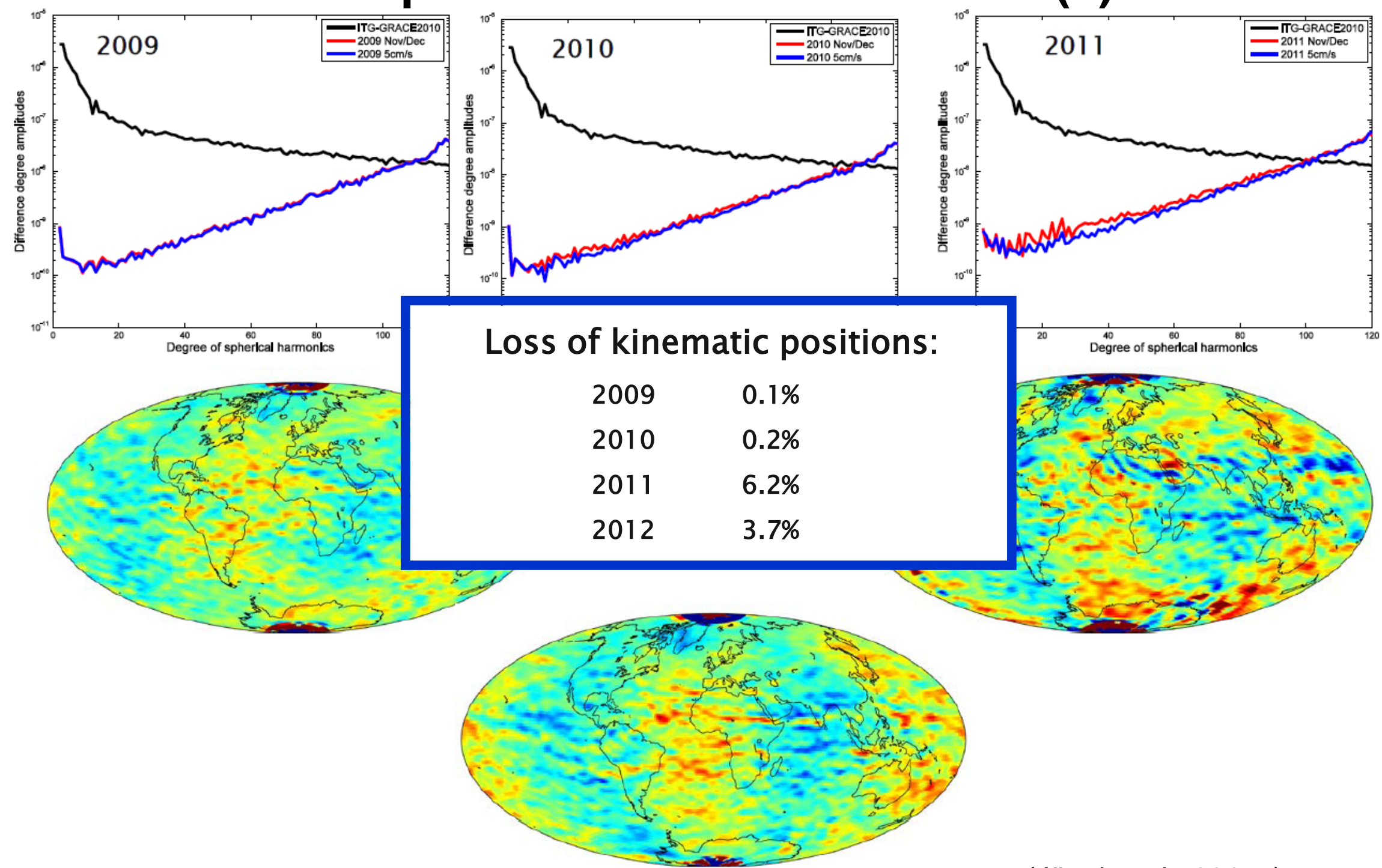

(Jäggi et al., 2015a) 


\section{Attempts to Model the lonospheric Effects (1)}

Conventional modeling of $\mathrm{HOI}$ correction terms does not show any improvements. Also the application of further $\mathrm{HOI}$ correction terms than recommended by the IERS Conventions 2010 does not bring any further improvements.

Ionosphere delays (= slant TEC) need to be directly derived from the geometryfree linear combination to compute more realistic $\mathrm{HOI}$ correction terms.
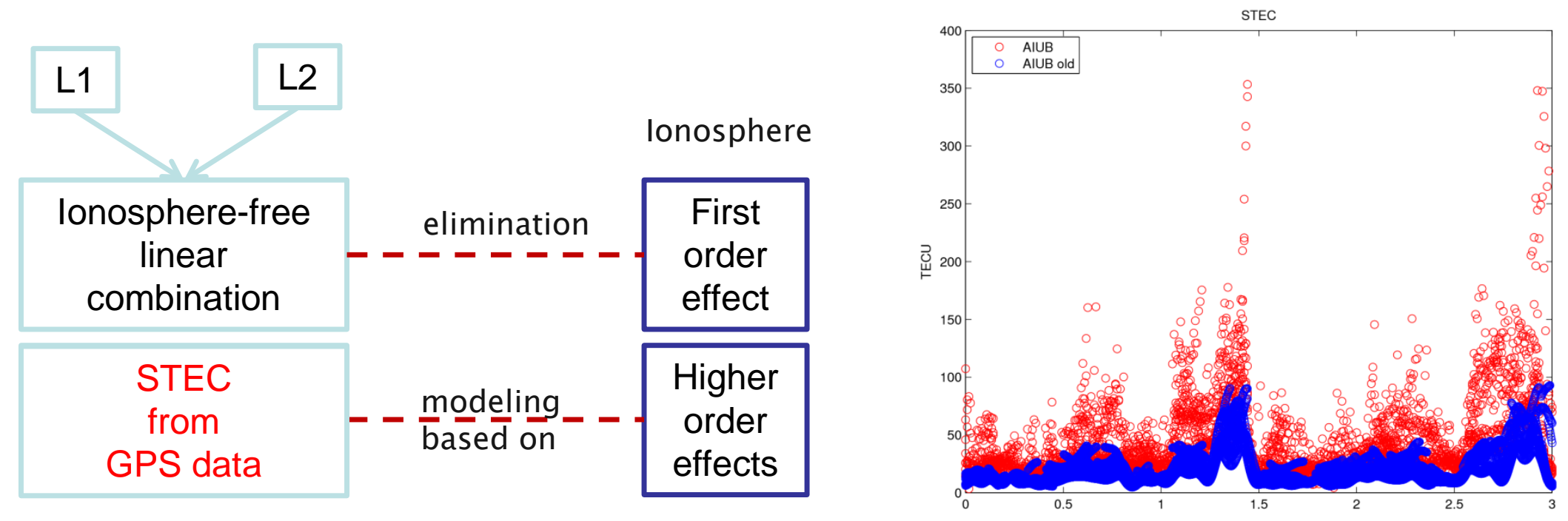


\section{Attempts to Model the Ionospheric Effects (2)}

STEC estimations are fed into the kinematic orbit determination instead of the global ionosphere map

$\mathrm{HOI}$ correction terms are computed based on the STEC estimations

Only partial reduction achieved so far in gravity field solutions

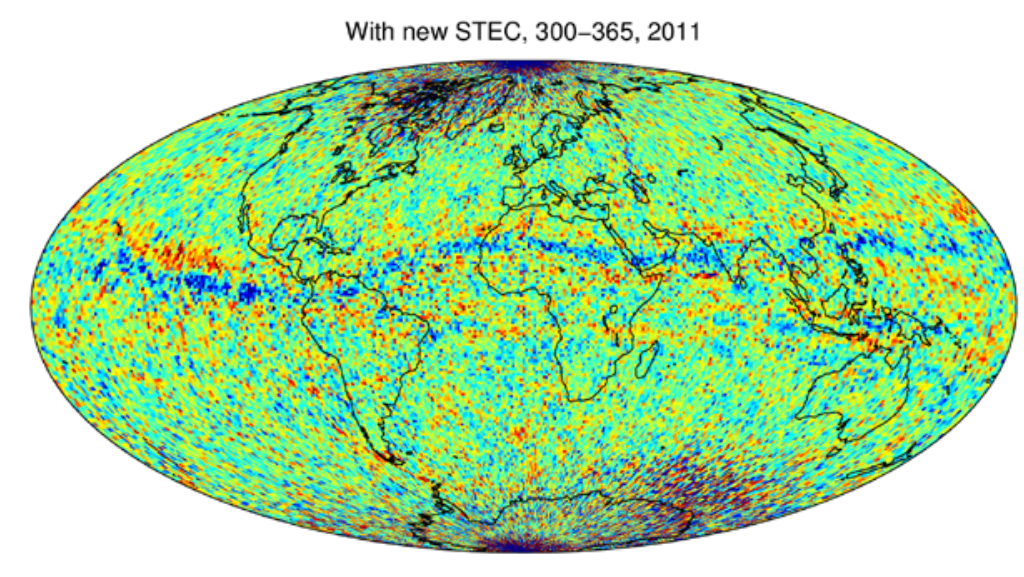

Phase observation residuals ($2 \mathrm{~mm}$... $+2 \mathrm{~mm}$ ) mapped to the ionosphere piercing point

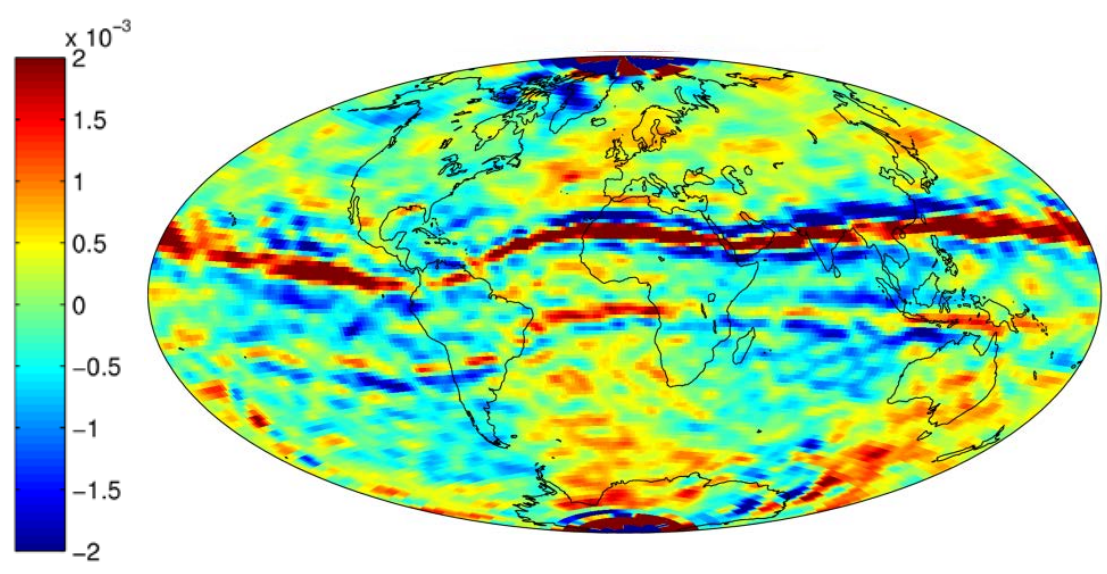

Geoid height differences $(-5 \mathrm{~cm}$... $5 \mathrm{~cm})$; Nov-

Dec 2011 


\section{Situation for other LEO Satellites (1)}

Original GPS Data (13 months)

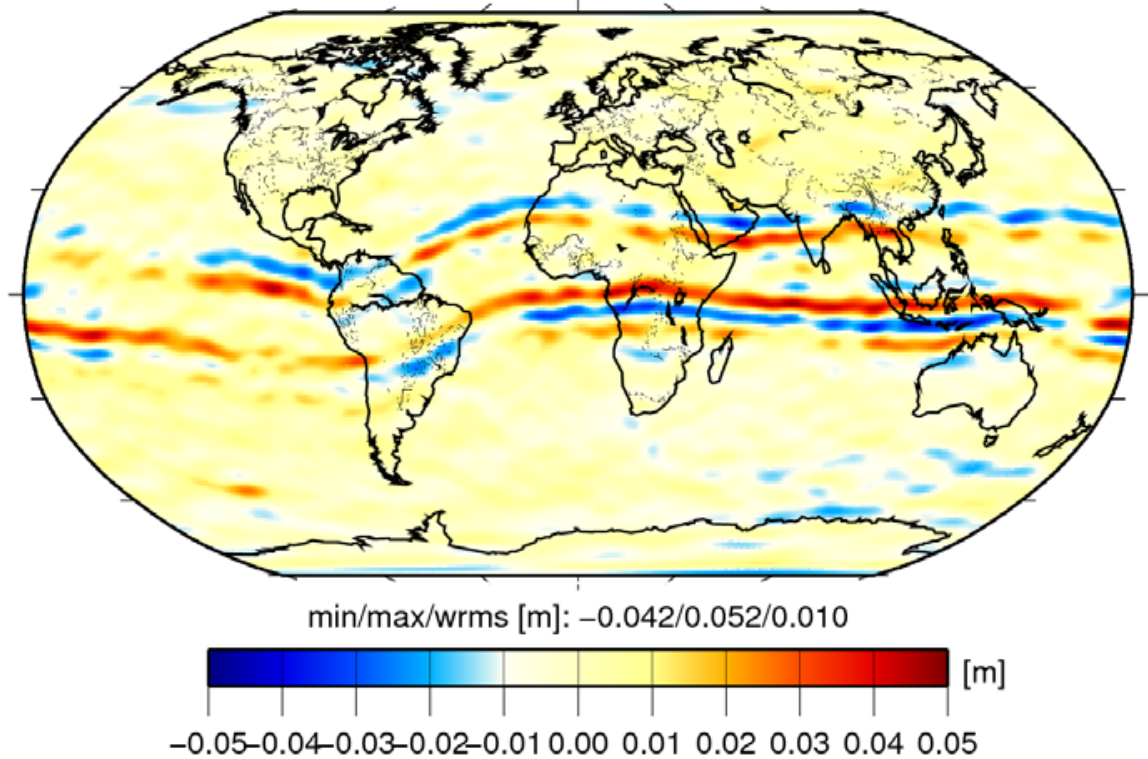

$-0.05-0.04-0.03-0.02-0.010 .000 .010 .020 .030 .040 .05$
Screened GPS Data (18 months)

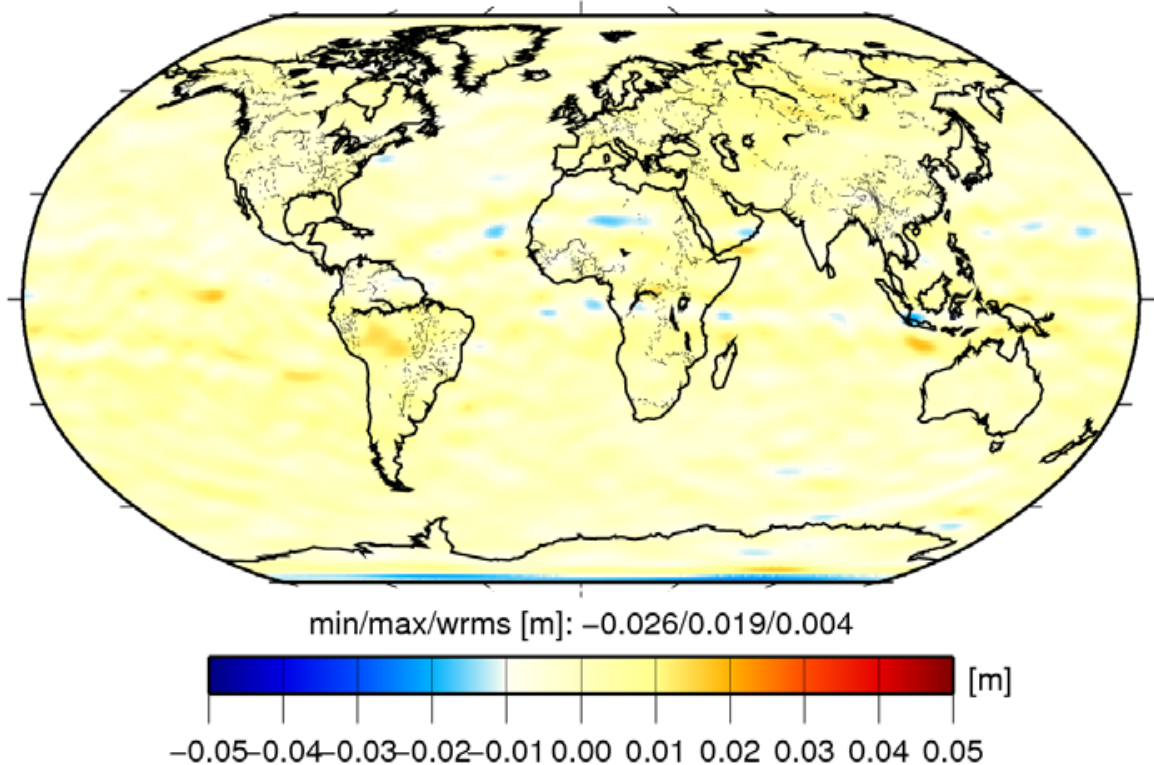

(Differences wrt GOCO05S, 400 km Gauss smoothing adopted)

Systematic signatures along the geomagnetic equator may be efficiently reduced for static Swarm gravity field recovery when screening the raw RINEX GPS data files with the dL4/dt criterion.

(Jäggi et al., 2015b) 


\section{Situation for other LEO Satellites (2)}

Original GPS Data

(Swarm)

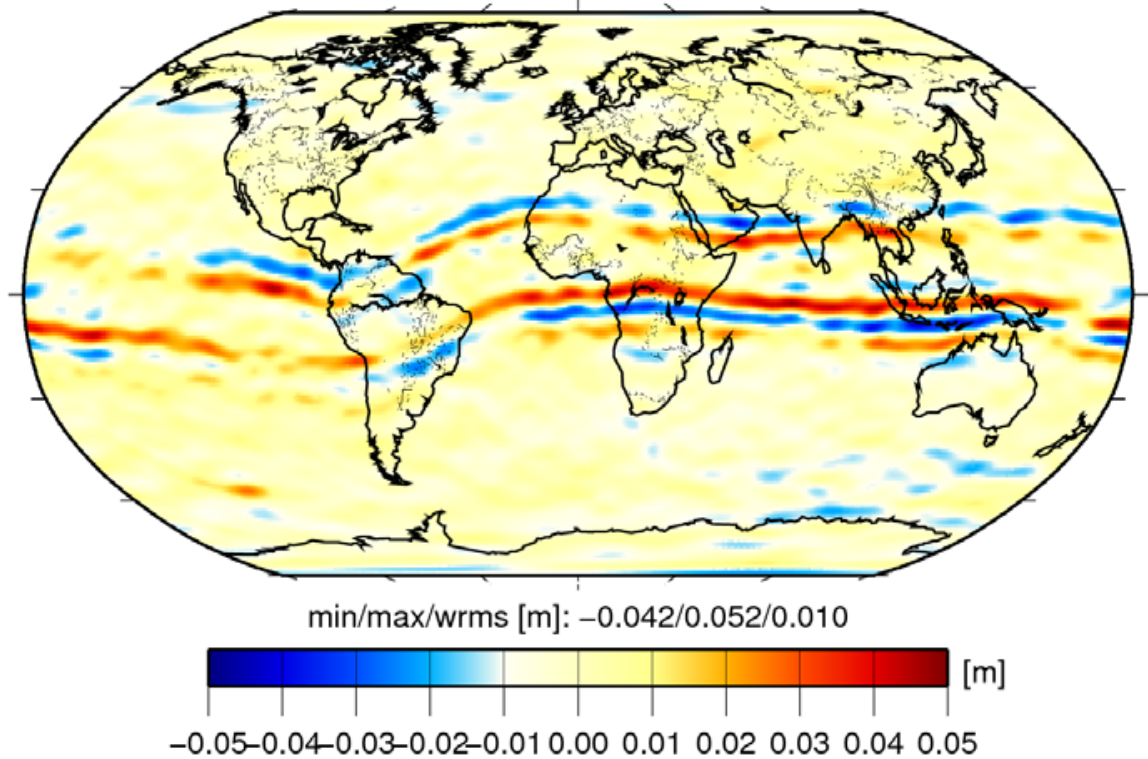

$-0.05-0.04-0.03-0.02-0.010 .000 .010 .020 .030 .040 .05$
Original GPS Data (GRACE)

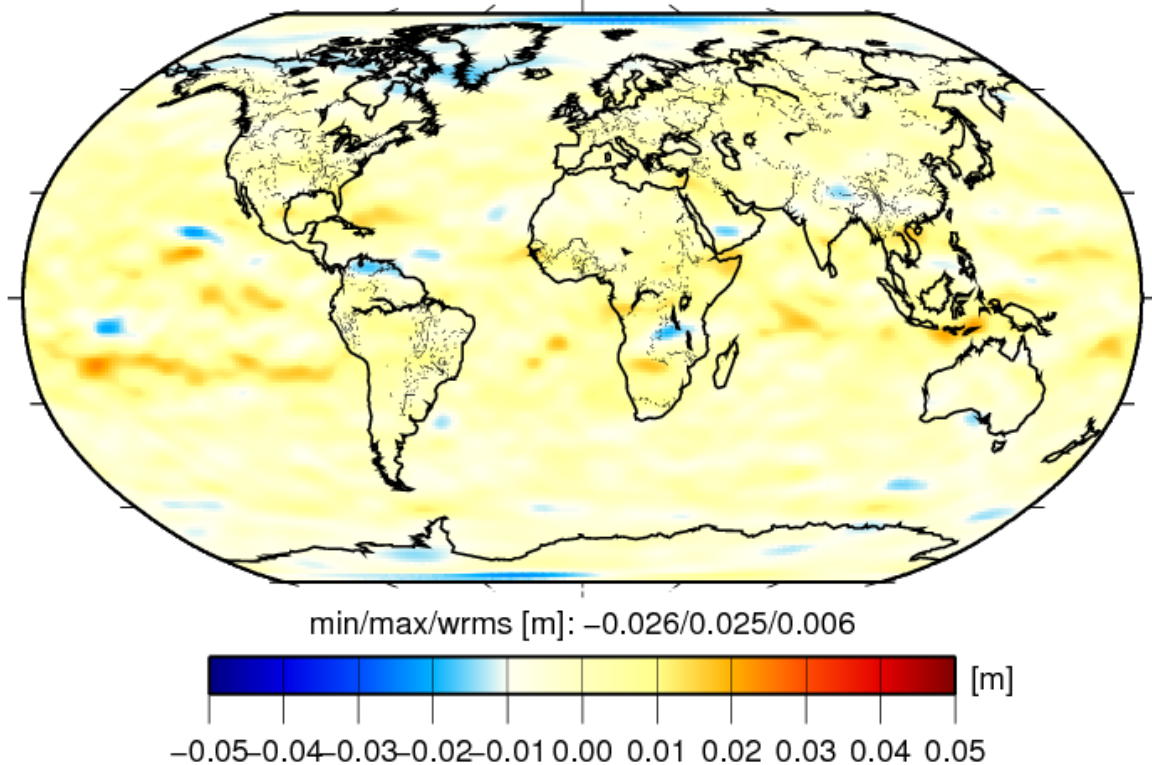

(Differences wrt GOCO05S, 400 km Gauss smoothing adopted)

Systematic signatures along the geomagnetic equator are not visible when using original L1B RINEX GPS data files from the GRACE mission. 


\section{Situation for other LEO Satellites (3)}

GRACE-B, doy 060-090, 2014 (all arcs)

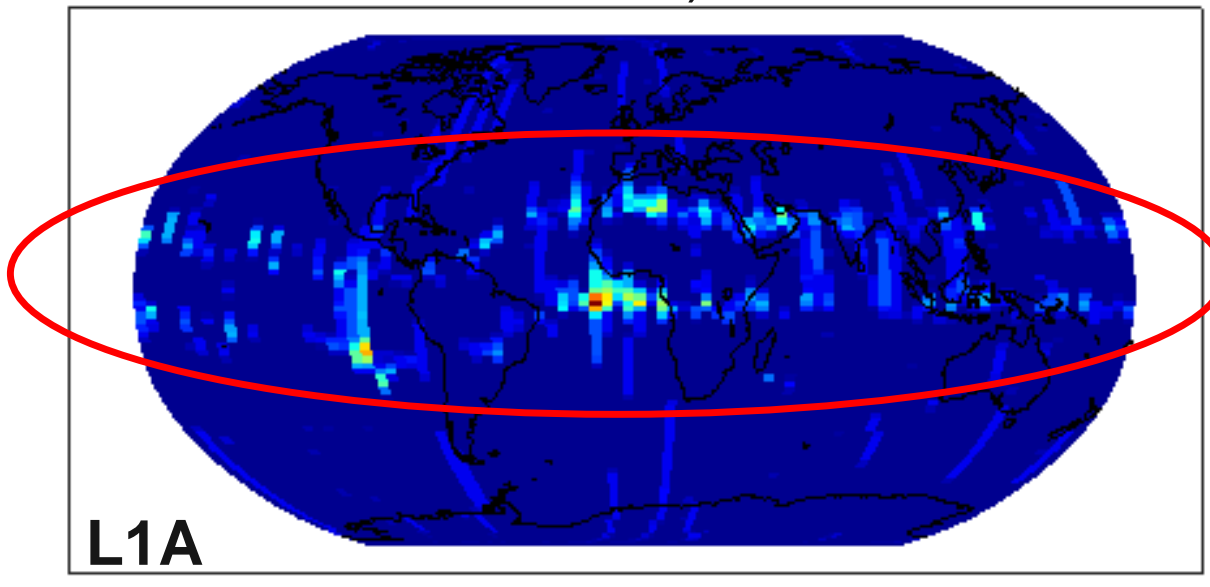

0

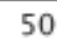

50

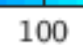

150

200

250

300
Swarm-A, doy 060-090, 2014 (all arcs)
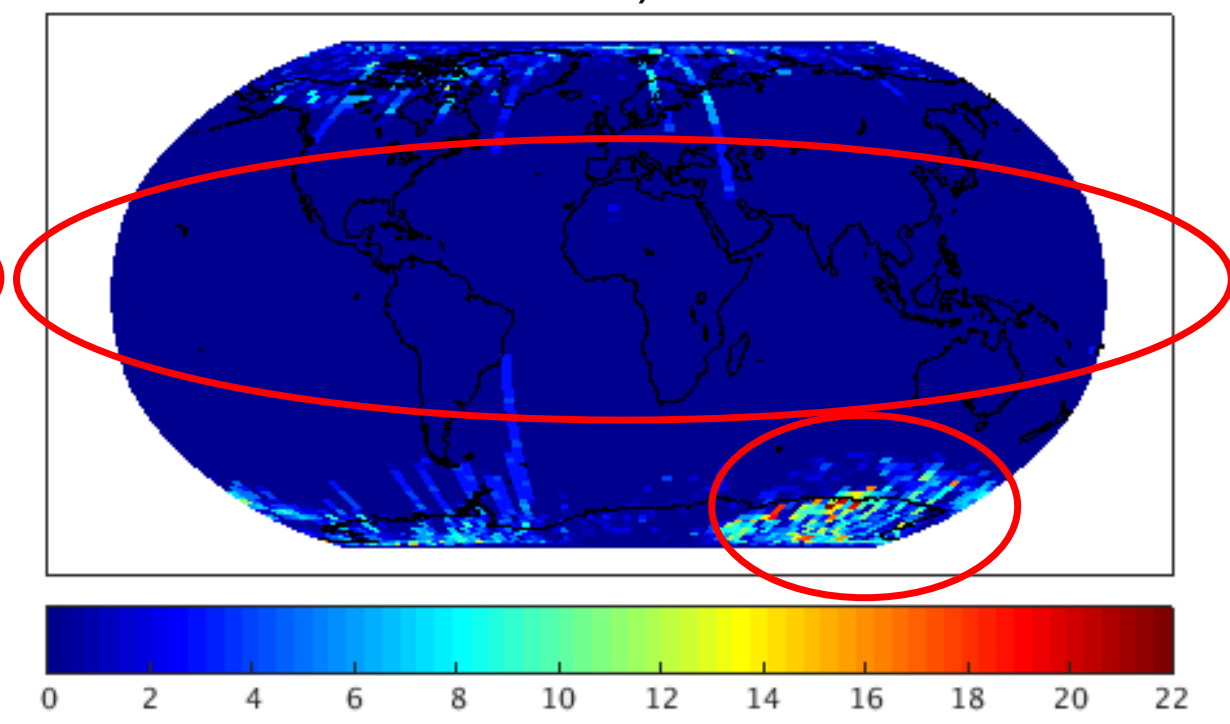

Significant amounts of data are missing in GRACE L1B RINEX files $=>$ problematic signatures cannot propagate into gravity field.

Swarm RINEX files are more complete (gaps only over the poles) $=>$ problematic signatures do propagate into the gravity field.

(Jäggi et al., 2015b) 


\section{Multi-Satellite SLR Solutions (1)}

\begin{tabular}{|c|c|}
\hline \multicolumn{2}{|c|}{ Estimated parameters } \\
\hline \multirow{3}{*}{$\frac{n}{0}$} & $\begin{array}{l}\text { Osculating } \\
\text { elements }\end{array}$ \\
\hline & $\begin{array}{l}\text { Dynamical } \\
\text { parameters }\end{array}$ \\
\hline & $\begin{array}{c}\text { Pseudo-stochastic } \\
\text { pulses }\end{array}$ \\
\hline \multicolumn{2}{|r|}{$\begin{array}{c}\text { Earth rotation } \\
\text { parameters }\end{array}$} \\
\hline \multicolumn{2}{|c|}{ Geocenter coordinates } \\
\hline \multicolumn{2}{|c|}{ Earth gravity field } \\
\hline \multicolumn{2}{|c|}{ Station coordinates } \\
\hline & ther parameters \\
\hline
\end{tabular}

\section{SLR solutions}

LAGEOS-1/2,

Starlette, Stella, AJISAI, LARES, Blits, Larets, Beacon-C

\begin{tabular}{c} 
a, e, $\mathrm{i}, \Omega, \omega, \mathrm{u}_{0}$ \\
(LAGEOS: 1 set per 10 days, \\
LEO: 1 set per 1 day) \\
LAGEOS-1/2: $\mathrm{S}_{0}, \mathrm{~S}_{\mathrm{S}}, \mathrm{S}_{\mathrm{C}}$ \\
(1 set per 10 days) \\
Sta/Ste/AJI $: \mathrm{C}_{\mathrm{D}}, \mathrm{S}_{\mathrm{C}}, \mathrm{S}_{\mathrm{S}}, \mathrm{W}_{\mathrm{C}}, \mathrm{W}_{\mathrm{S}}$ \\
(1 set per day) \\
LAGEOS-1/2 : no pulses \\
Sta/Ste/AJI : once-per-revolution \\
in along-track only \\
$\mathrm{X}_{\mathrm{P}}, \mathrm{Y}_{\mathrm{P}}, \mathrm{UT} 1-\mathrm{UTC}$ \\
(Piecewise linear, 1 set per day) \\
\hline 1 set per 30 days
\end{tabular}

\section{Estimated up to $\mathrm{d} / \mathrm{o}$ 10/10} (1 set per 30 days)

1 set per 30 days
Range biases for all stations (LEO)
and for selected stations (LAGEOS)

LAGEOS-1

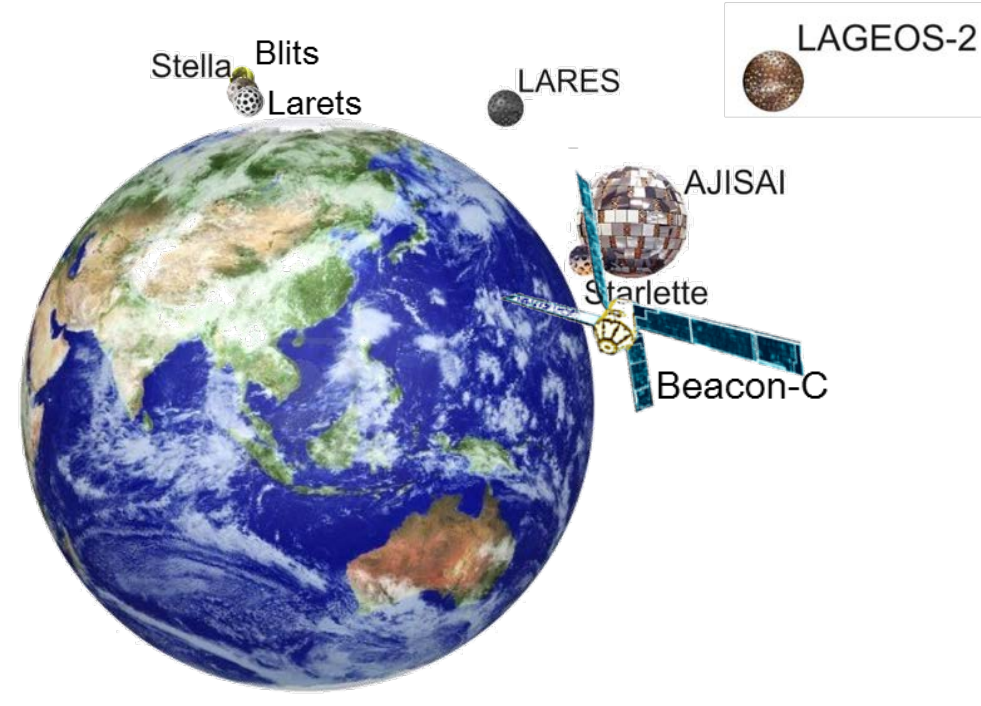

Up to 9 SLR satellites with different altitudes and different inclinations are used.

For LAGEOS-1/2: 10-day arcs are generated, for low orbiting satellites: 1-day arcs.

Different weighting of observations is applied: from $8 \mathrm{~mm}$ for LAGEOS-1/2 to $50 \mathrm{~mm}$ for Beacon-C.

Constraints introduced to regularize the normal equations (on GFC, pulses, EOPs).

(Sosnica, 2015) 


\section{Multi-Satellite SLR Solutinng (2)}

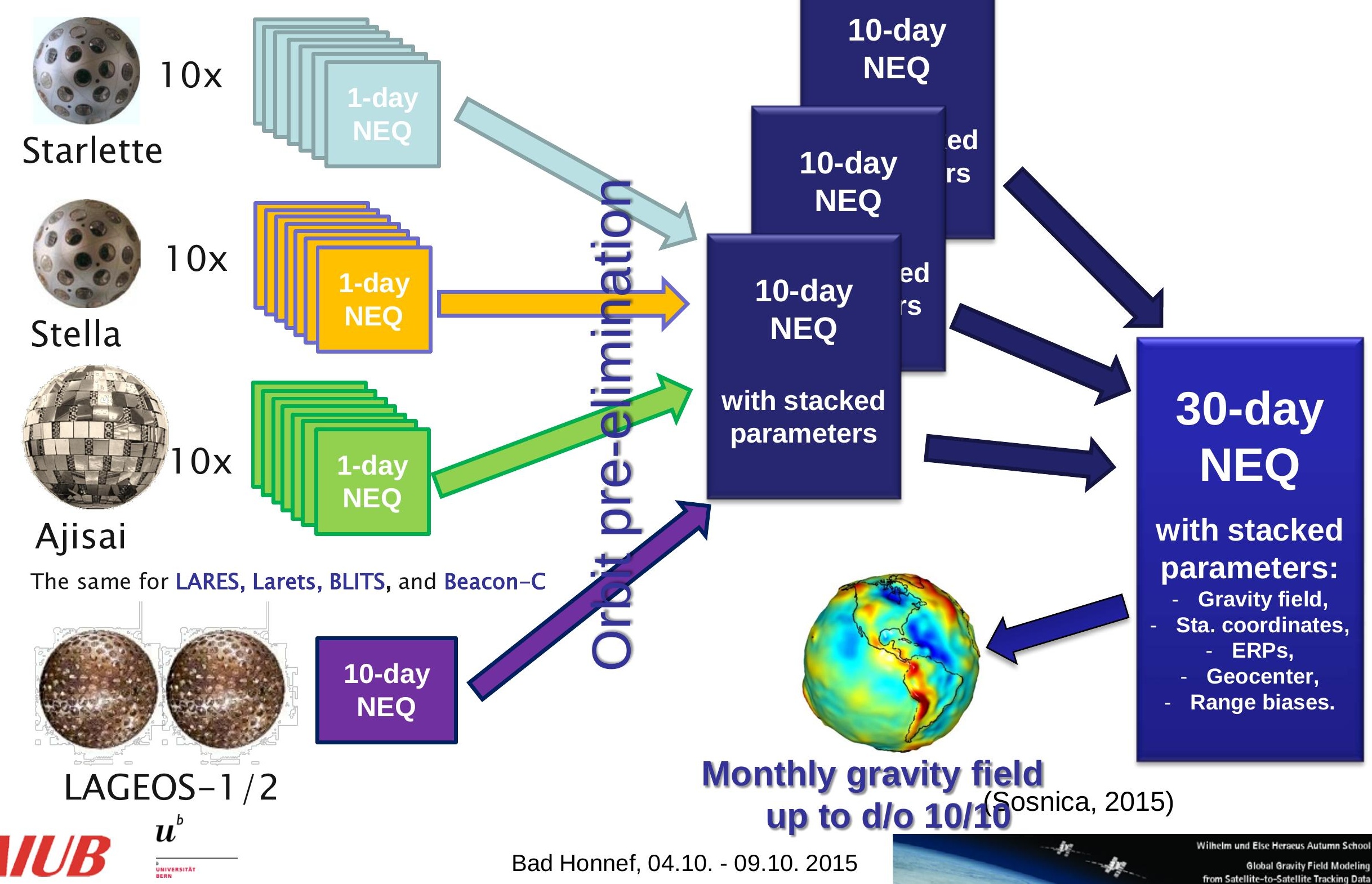




\section{Multi-Satellite SLR Solution (3)}

Table 5.9: Impact of different orbit parameterizations of LEO satellites on a posteriori sigma of unit weight and ERP (comparison w.r.t. IERS-08-C04).

\begin{tabular}{|c|c|c|c|c|c|c|c|c|c|c|c|}
\hline \multirow[t]{2}{*}{ Sol } & \multirow{2}{*}{$\begin{array}{l}\text { Length } \\
\text { of sol. } \\
\text { [days] }\end{array}$} & \multirow{2}{*}{$\begin{array}{l}\text { Sets of } \\
\text { oscul. } \\
\text { elem. }\end{array}$} & \multirow{2}{*}{$\begin{array}{l}\text { Sets of } \\
\text { dyn. } \\
\text { par. }\end{array}$} & \multirow{2}{*}{$\begin{array}{l}\text { Stoch. } \\
\text { pulses }\end{array}$} & \multirow{2}{*}{$\begin{array}{l}\text { RMS } \\
\text { resid } \\
{[\mathrm{mm}]}\end{array}$} & \multicolumn{2}{|c|}{ X pole } & \multicolumn{2}{|c|}{ Y pole } & \multicolumn{2}{|c|}{ LoD } \\
\hline & & & & & & $\begin{array}{r}\text { bias } \\
{[\mu \mathrm{as}]}\end{array}$ & $\begin{array}{r}\text { WRMS } \\
{[\mu \mathrm{as}]}\end{array}$ & $\begin{array}{r}\text { bias } \\
{[\mu \mathrm{as}]}\end{array}$ & $\begin{array}{r}\text { WRMS } \\
{[\mu \mathrm{as}]}\end{array}$ & $\begin{array}{r}\text { bias } \\
{[\mu \mathrm{s} / \mathrm{d}]}\end{array}$ & $\begin{array}{r}\text { WRMS } \\
{[\mu \mathrm{s} / \mathrm{d}]}\end{array}$ \\
\hline A & 7 & 1 & 7 & $\mathrm{~S}$ & 7.78 & 57.7 & 269.8 & -8.7 & 218.1 & -3.6 & 106.5 \\
\hline B1 & 7 & 1 & 1 & $\mathrm{~S}$ & 13.50 & 38.6 & 508.7 & -6.8 & 442.3 & -15.0 & 102.2 \\
\hline B2 & 7 & 7 & 7 & S & 13.42 & 20.7 & 395.7 & 4.4 & 400.1 & -2.2 & 120.0 \\
\hline $\mathrm{C} 1$ & 7 & 1 & 7 & $\mathrm{~S}, \mathrm{R}, \mathrm{W}$ & 7.52 & 57.7 & 269.8 & -8.7 & 218.1 & -3.7 & 116.5 \\
\hline $\mathrm{C} 2$ & 7 & 1 & 7 & - & 7.81 & 85.5 & 350.2 & 0.1 & 275.7 & -36.3 & 140.4 \\
\hline D1 & 6 & 1 & 2 & $\mathrm{~S}$ & 8.21 & 25.7 & 282.6 & 2.4 & 254.2 & -25.4 & 119.7 \\
\hline D2 & 6 & 1 & 3 & $\mathrm{~S}$ & 7.98 & 28.2 & 280.7 & 10.5 & 244.8 & -13.5 & 115.1 \\
\hline D3 & 6 & 1 & 6 & $\mathrm{~S}$ & 7.65 & 32.1 & 270.5 & -4.3 & 217.9 & -6.7 & 105.8 \\
\hline
\end{tabular}

SLR only provides a sparse coverage of the orbits. In order to provide solutions of good quality, most dynamic solutions must be generated, e.g., by using long Arcs for the high orbiting LAGEOS satellite. Nevertheless, model deficiencies for the low orbiting satellites, e.g., due to air drag, need to be compensated by a small number of pseudo-stochastic parameters.

(Sosnica et al., 2014a) 


\section{Multi-Satellite SLR Solution (4)}

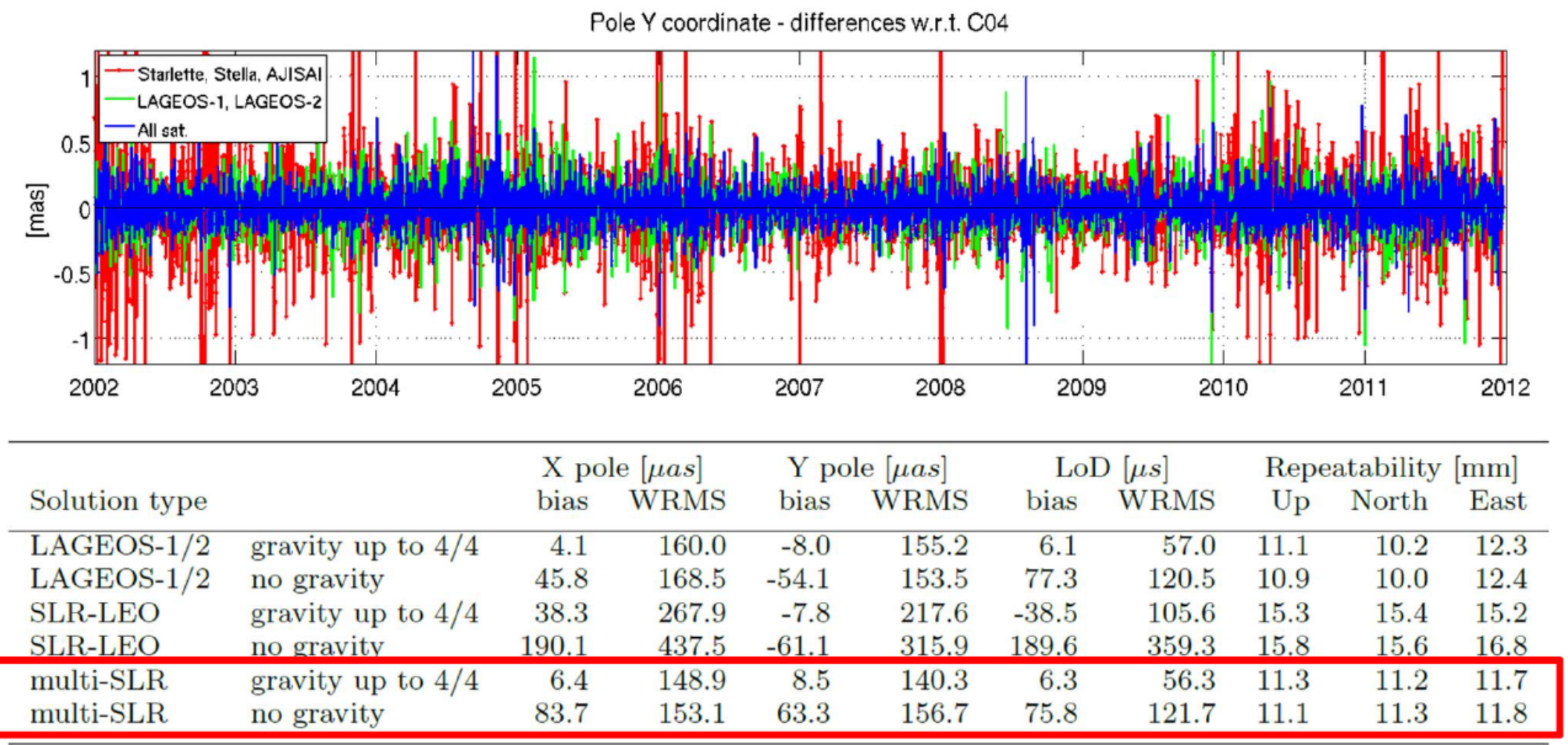

SLR orbits are difficult to validate. The quality of the geophysical parameters of interest, which are co-estimated in the frame of the generalized orbit determination problem, provide the basis to assess the quality of the solution. Best results are obtained for a multi-satellite solution. 


\section{Multi-Satellite SLR Solution (5)}
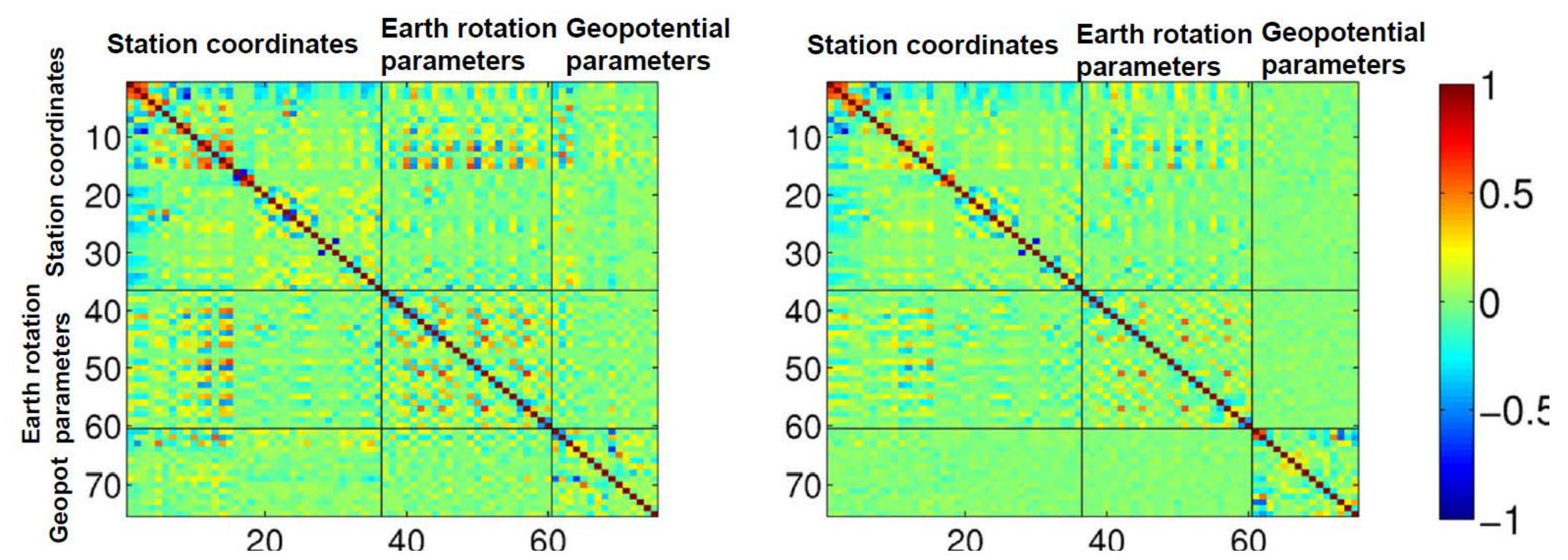

Multi-satellite solutions provide the advantage that the correlations between the estimated parameters (ERPs, geopotential coefficients, station coordinates) can be substantially reduced (better observation geometry due to the different orbital characterstics).

(Sosnica et al., 2014a) 


\section{Time-Variable Gravity from Non-Dedicated Satellites}

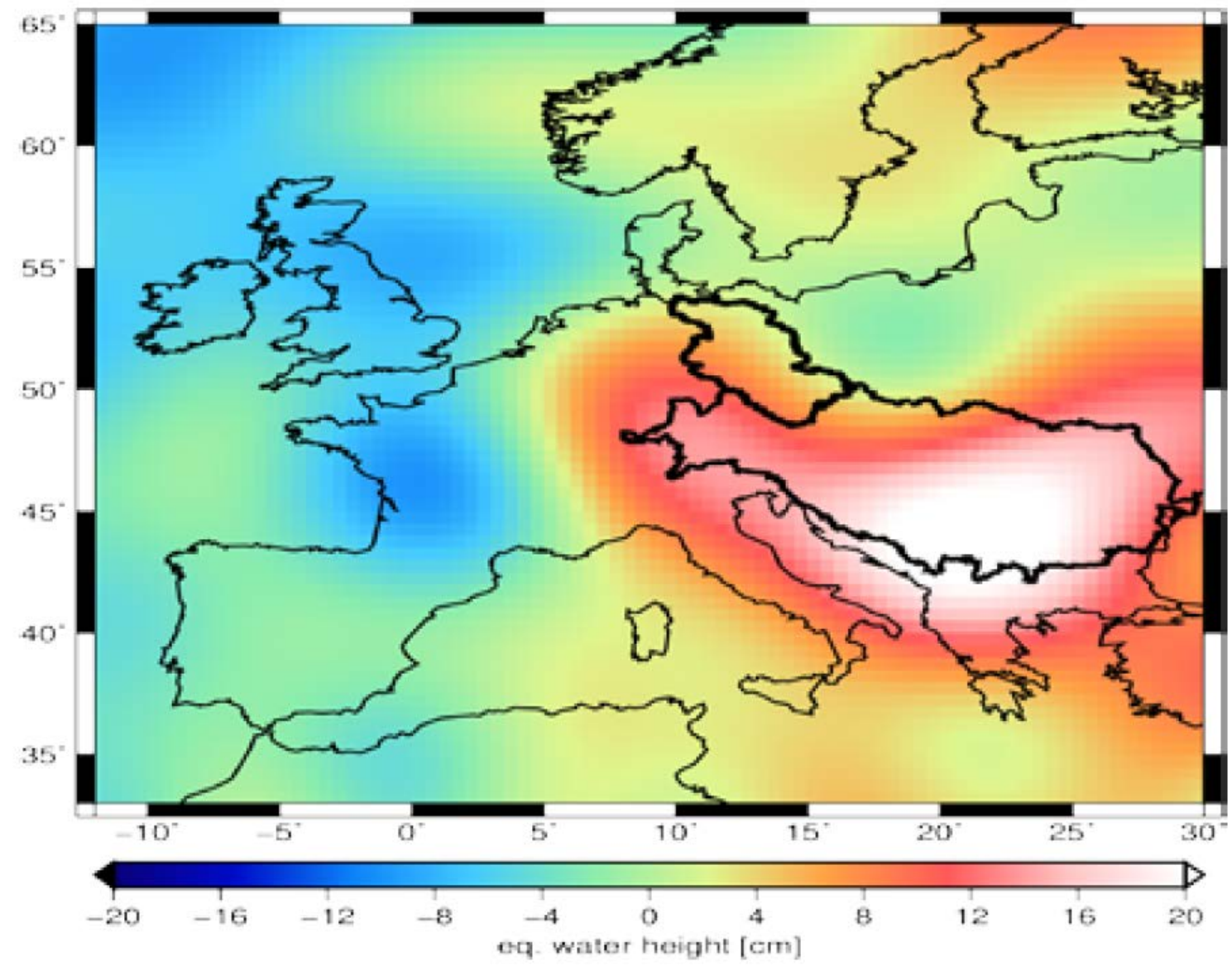

How well can time-variability by monitored by non-dedicated satellites tracked by SLR and GPS hl-SST? 


\section{Time-Variable Gravity from Non-Dedicated Satellites: SLR}
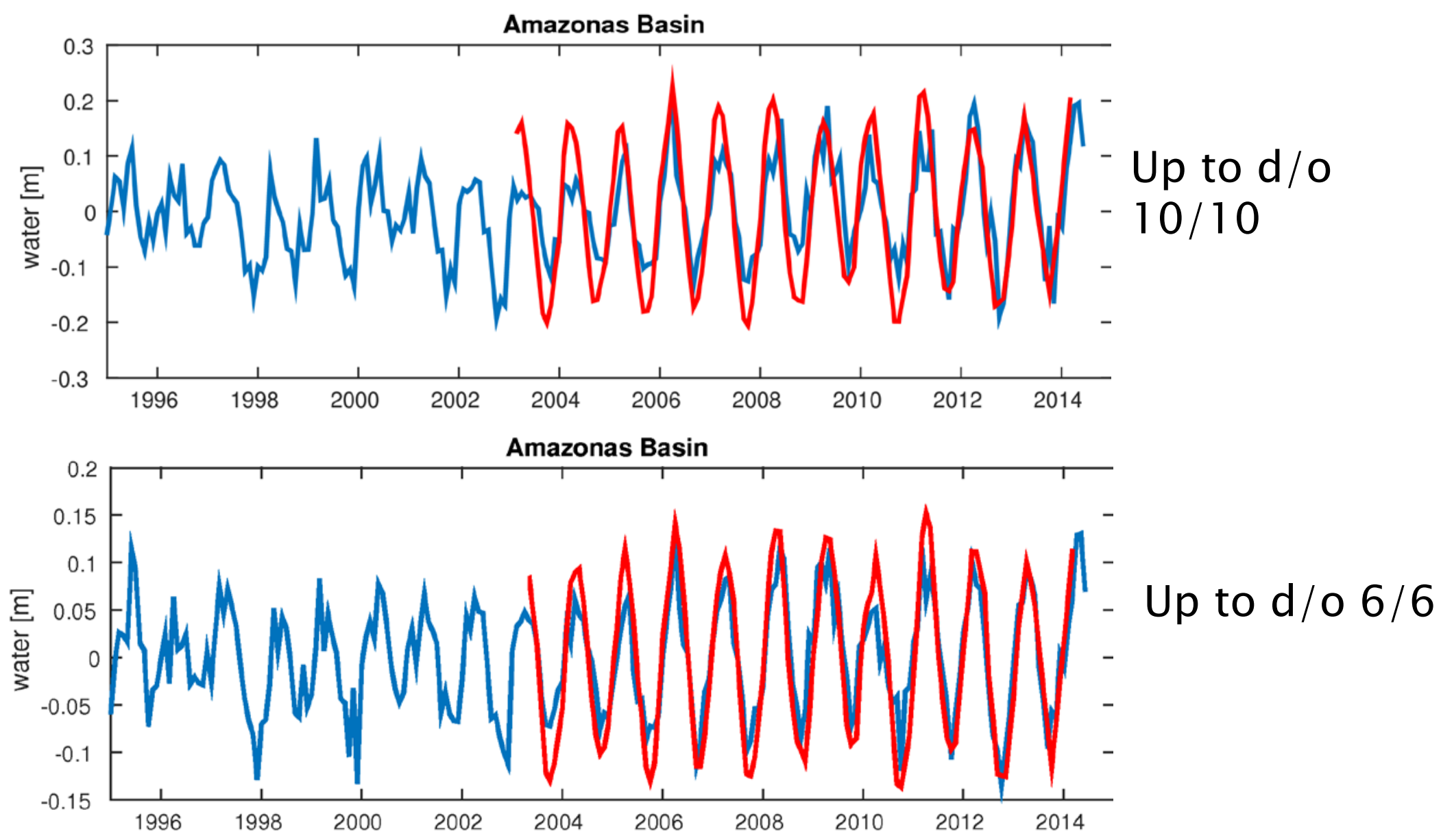

(Sosnica et al., 2015b) 


\section{Time-Variable Gravity from Non-Dedicated Satellites: SLR}
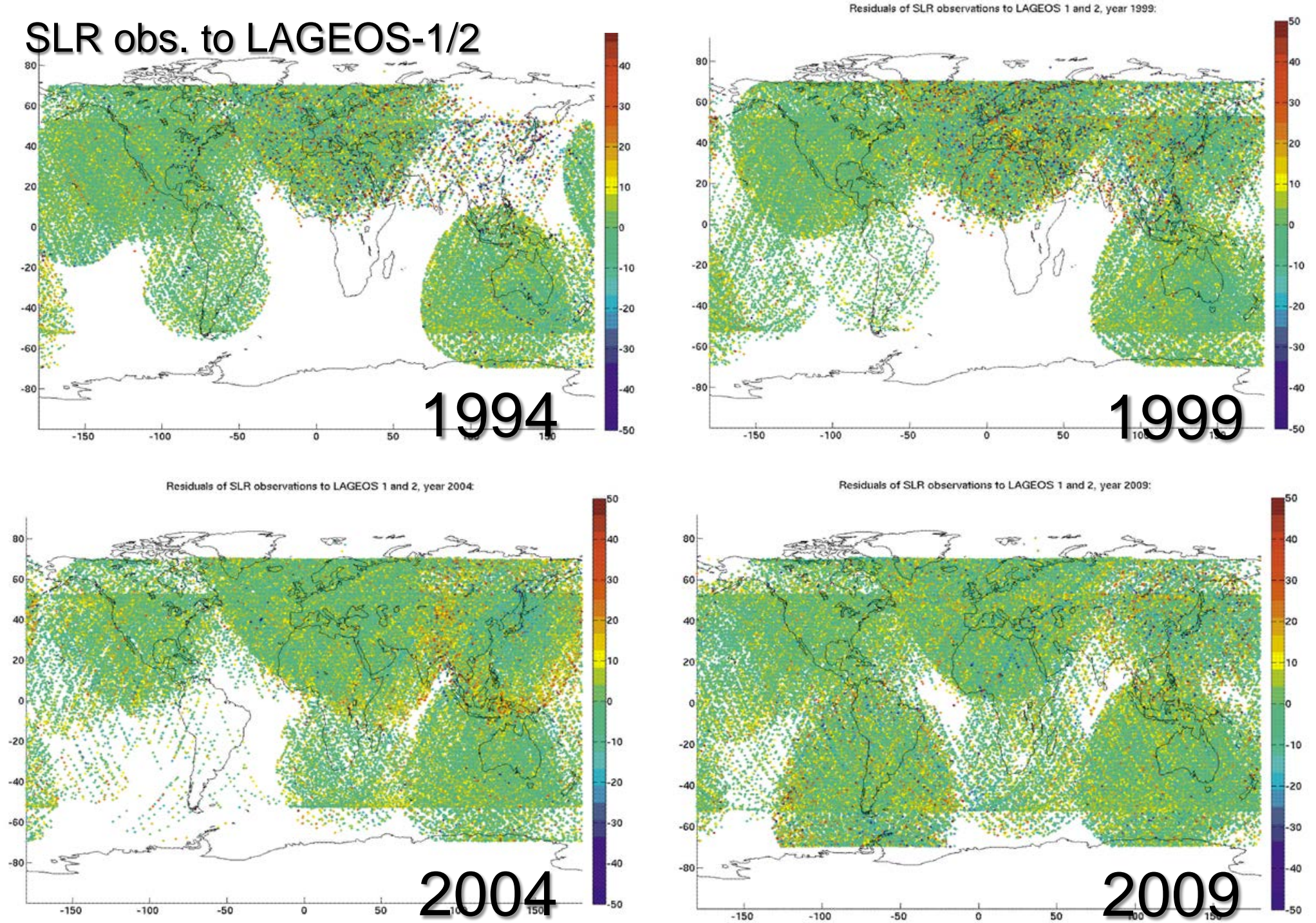


\section{Time-Variable Gravity from Non-Dedicated Satellites: SLR}

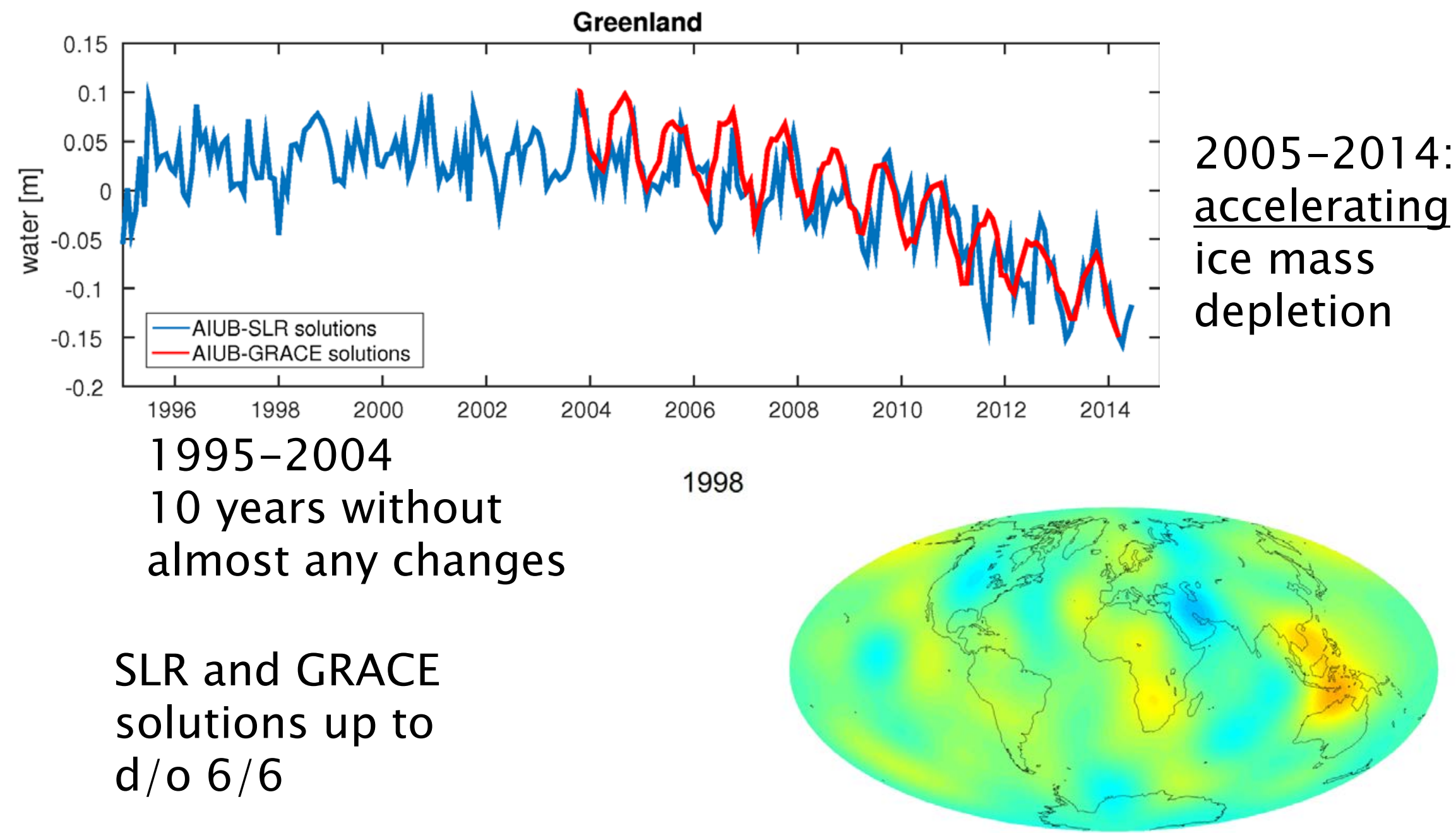

(Sosnıca et al., 2015b) 


\section{Time-Variąble Gravity from Non-Dedicated Satellites: GPS}
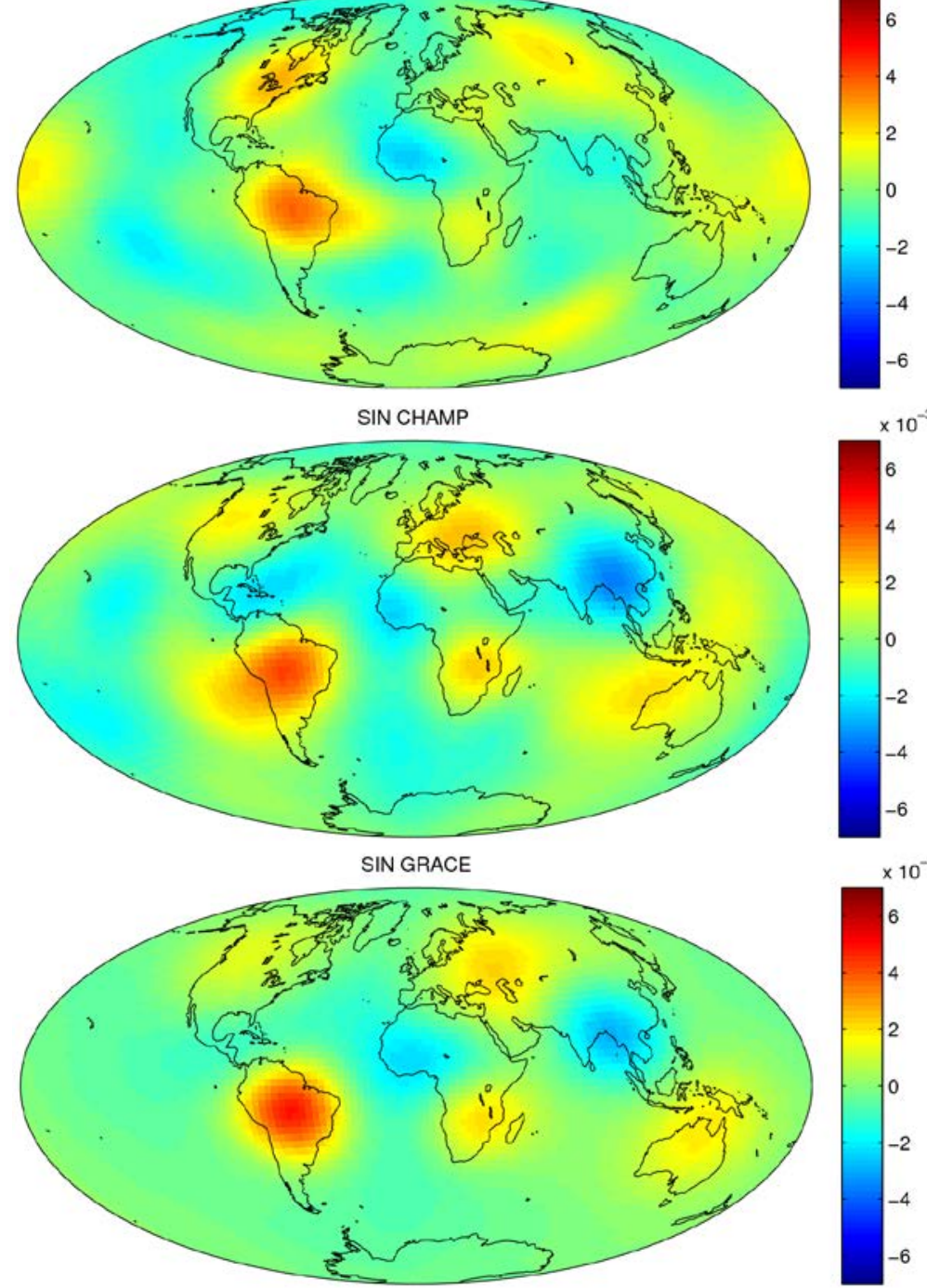
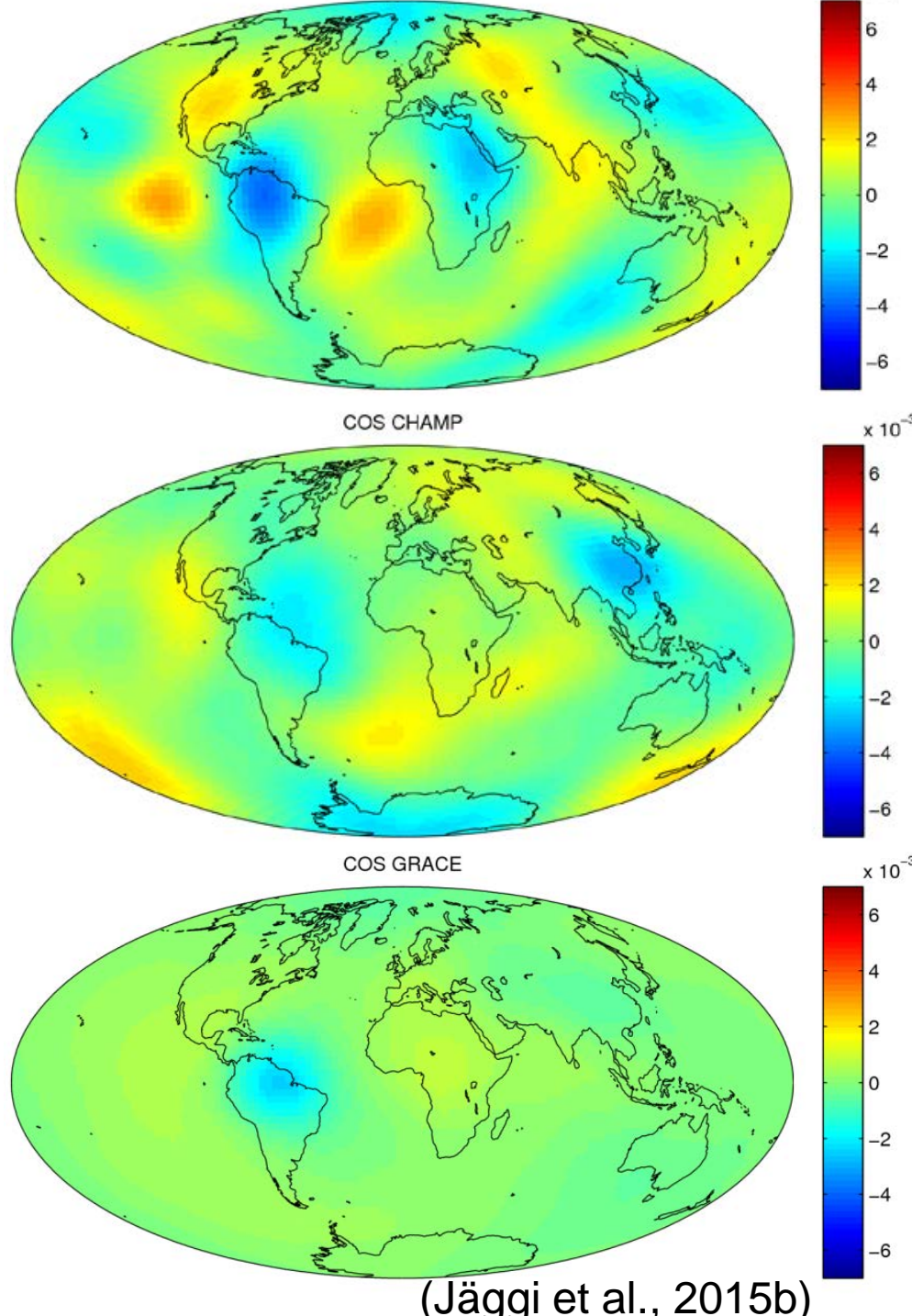


\section{Time-Variable Gravity from Non-Dedicated Satellites: Swarm}
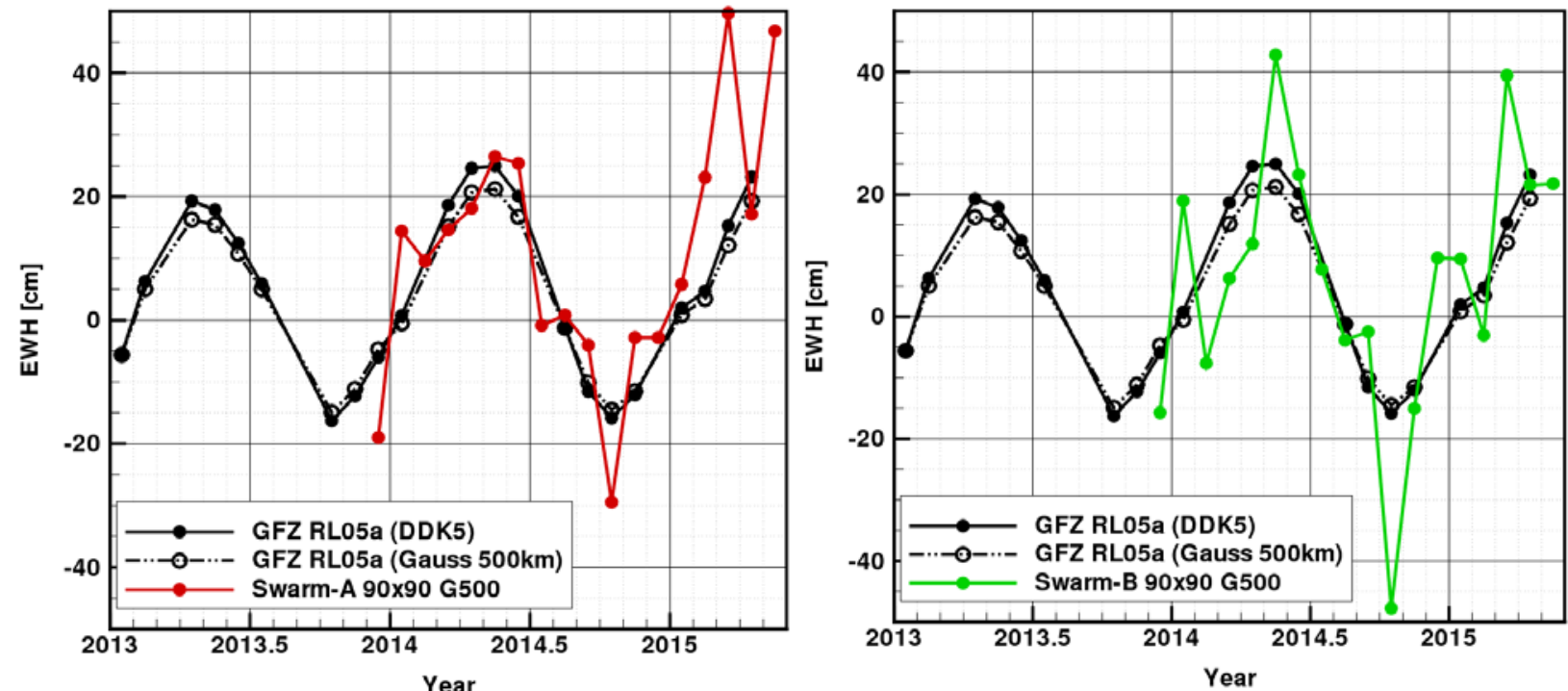

"True" signal:

- GFZ-RL05a (DDK5-filtered)

"Comparison" signal:

- GFZ-RL05a (500km Gauss)
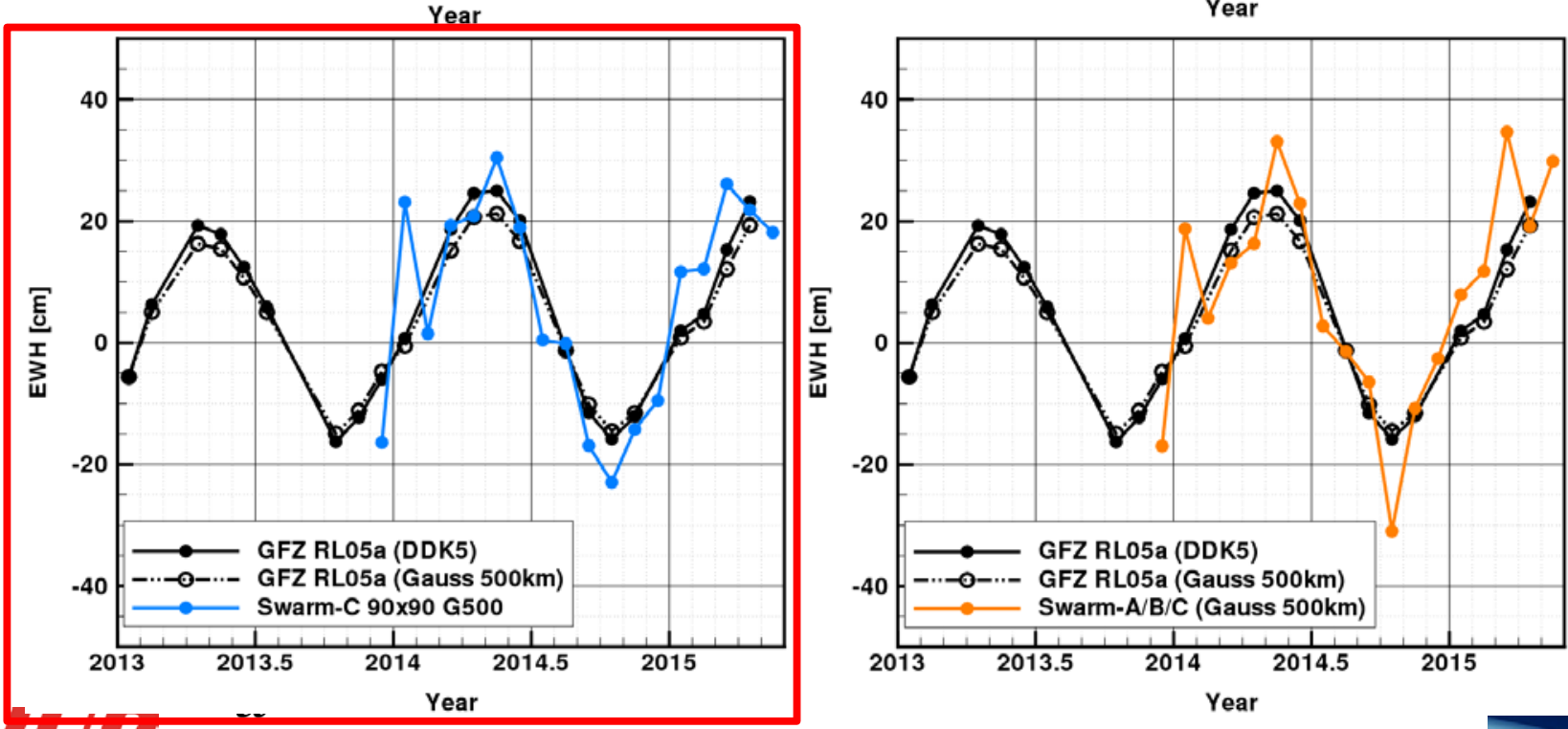

Swarm signal:

- $90 \times 90$ solutions (Gauss-filtered)

Result:

- Best agreement for Swarm-C

(Jäggi et al., 2015b)

Bad Honnef, 04.10. - 09.10. 2015 


\section{Time-Variable Gravity from Non-Dedicated Satellites: Combo}

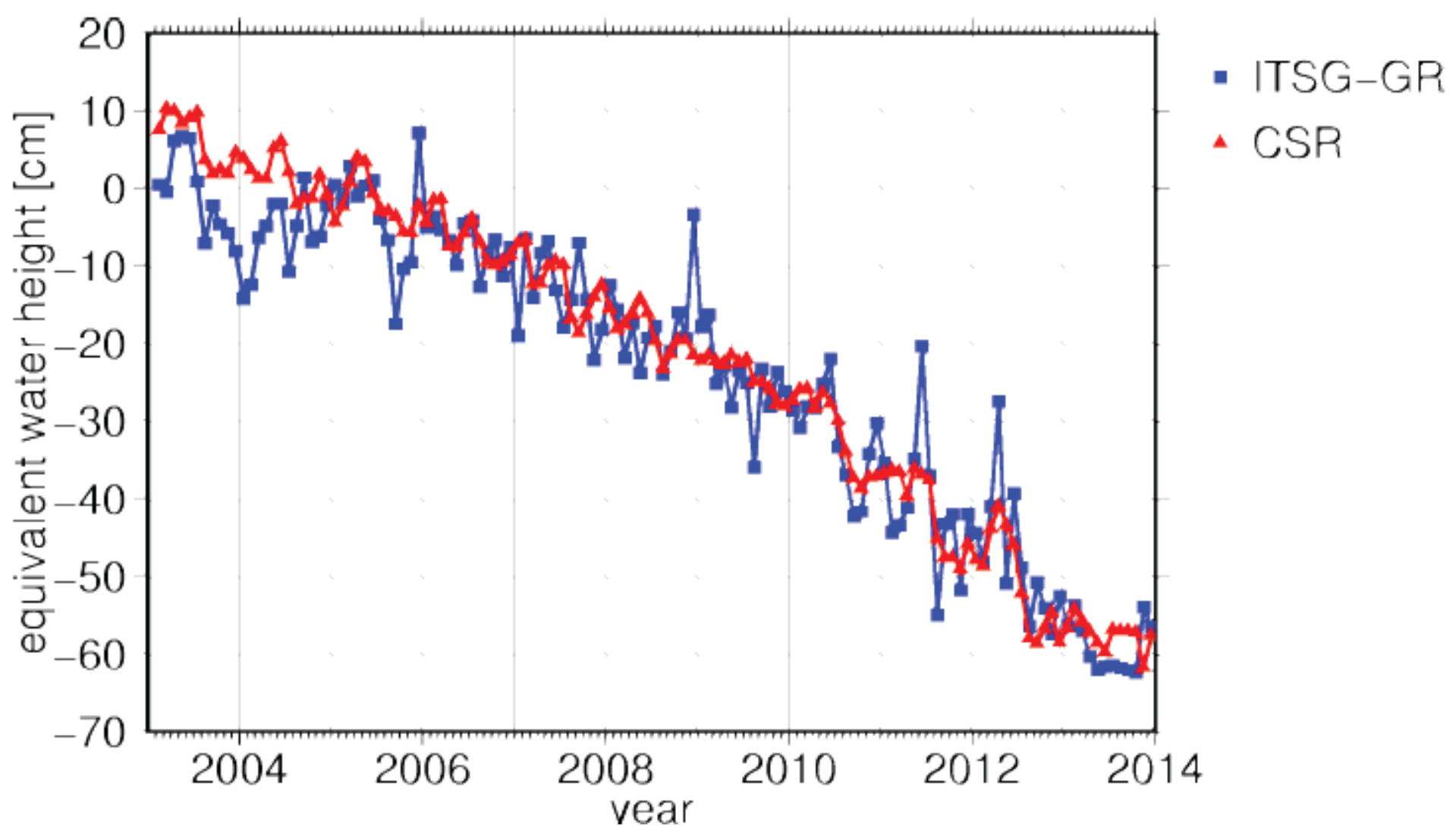

Combination of a multitude of LEO satellites tracked by GPS hl-SST provides Promising recoveries also for smaller signals.

(Zehentner et al., 2015) 


\section{Time-Variable Gravity from Non-Dedicated Satellites: Combo}
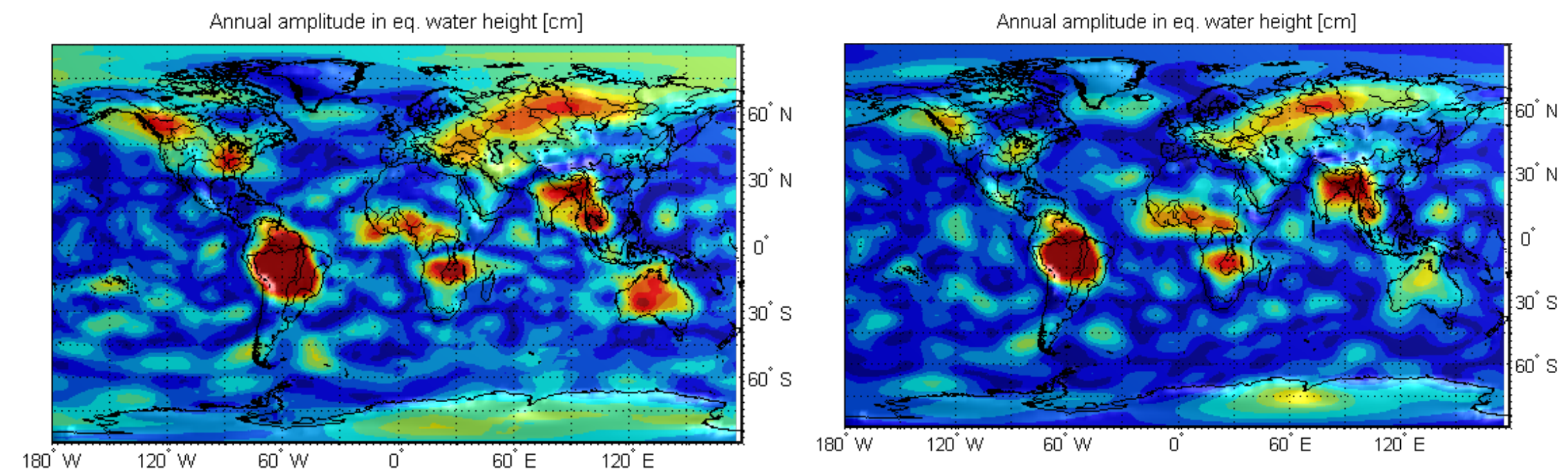

Annual amplitude in eq. water height [cm]
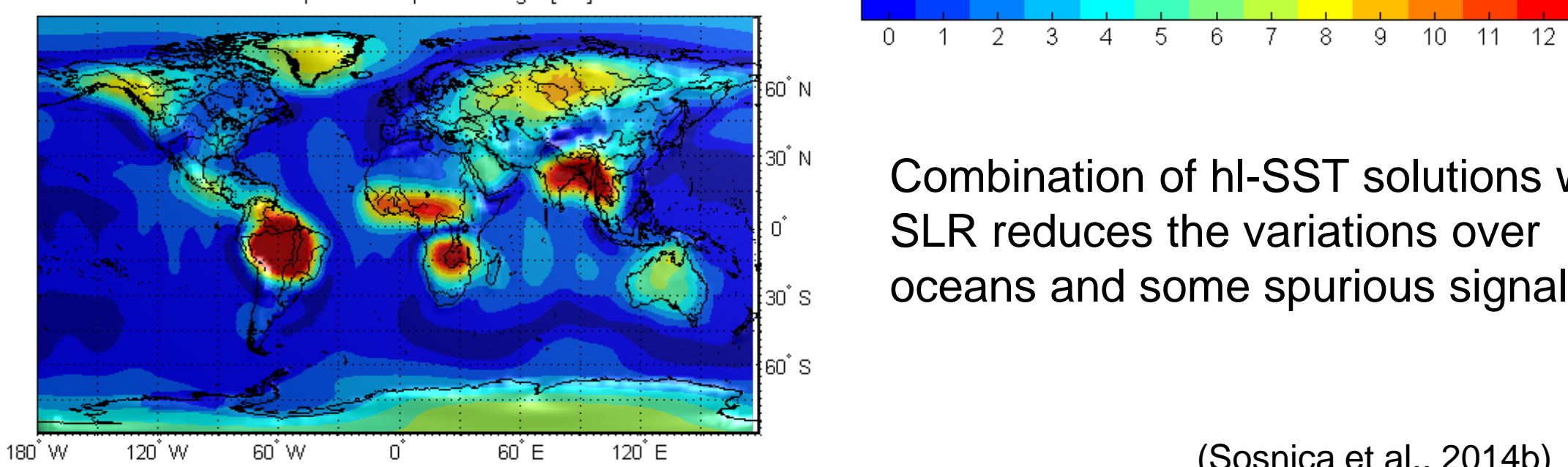

Combination of hl-SST solutions with SLR reduces the variations over oceans and some spurious signals.

(Sosnica et al., 2014b) 


\section{Time-Variable Gravity from Non-Dedicated Satellites: Combo}
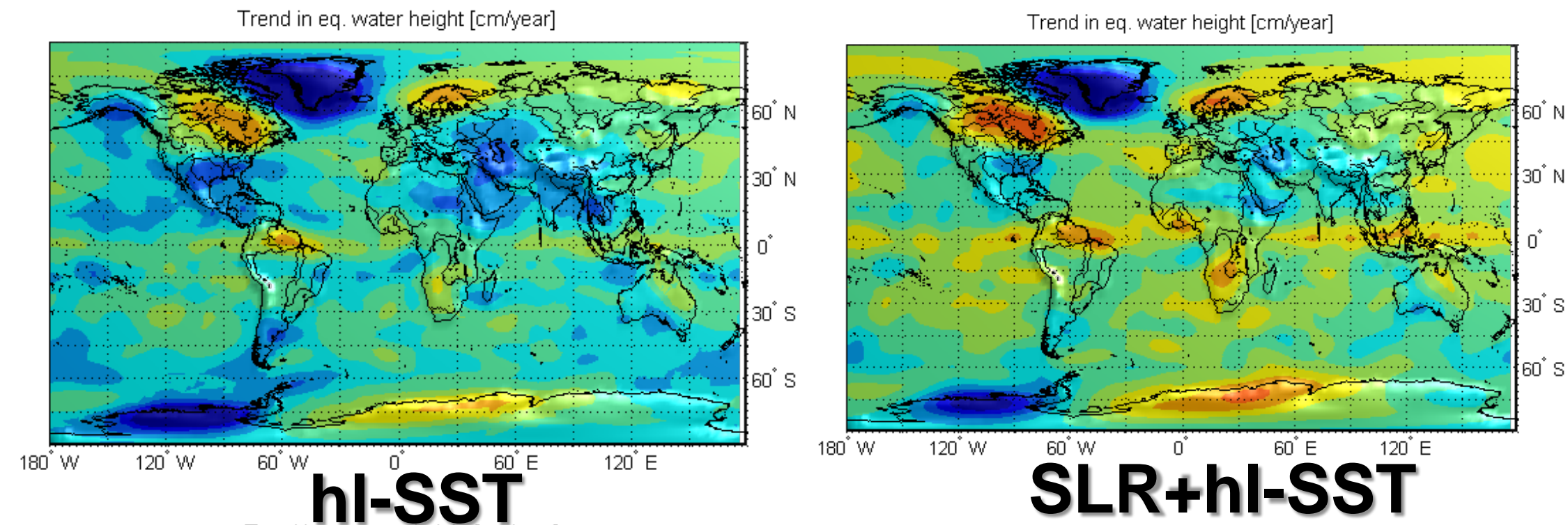

Trend in eq. water height [cm/year]
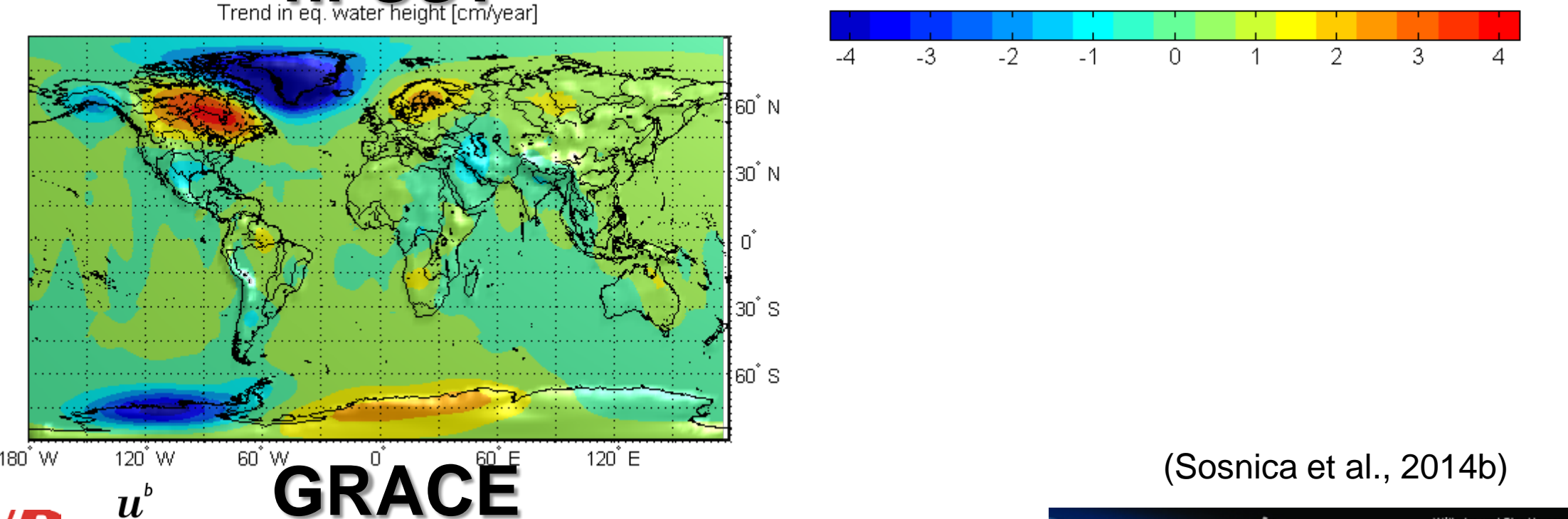

(Sosnica et al., 2014b) 


\section{Thank you for your attention}

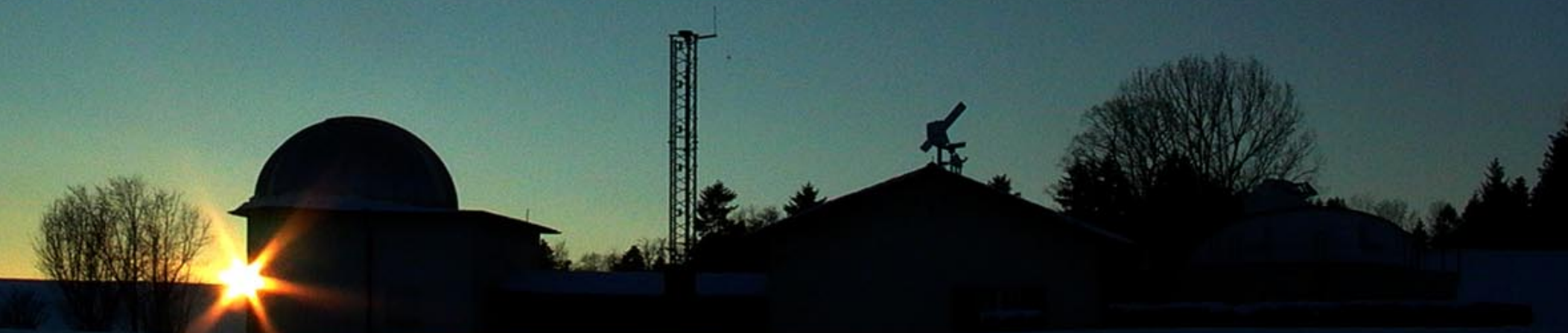




\section{Literature (1)}

Beutler, G. (2005) Methods of Celestial Mechanics. Vol 1: Physical, Mathematical, and Numerical Principles. Springer, ISBN 3-540-40749-9

Blewitt, G. (1997): Basics of the GPS Technique: Observation Equations, in Geodetic Applications of GPS, Swedish Land Survey, pp. 10-54, available at http://www.sbg.ac.at/mat/staff/revers/lectures/2006_2007/GPS/ GPSBasics.pdf

Bigazzi, A., B. Frommknecht (2010): Note on GOCE instruments positioning, XGCE-GSEG-EOPG-TN-09-0007, Issue 3.1, European Space Agency, available at http://earth.esa.int/download/goce/GOCE-LRR-GPSpositioning-Memo_3.1_[XGCE-GSEG-EOPG-TN-09-0007\%20v3.1].pdf Bock, H., R. Dach, A. Jäggi, G. Beutler (2009): High-rate GPS clock corrections from CODE: Support of $1 \mathrm{~Hz}$ applications. Journal of Geodesy, 83(11), 1083-1094, doi: 10.1007/s00190-009-0326-1

Bock, H., A. Jäggi, G. Beutler, U. Meyer (2014): GOCE: Precise orbit determination for the entire mission. Journal of Geodesy, 88(11), 10471060, doi: 10.1007/s00190-014-0742-8 


\section{Literature (2)}

Dach, R., E. Brockmann, S. Schaer, G. Beutler, M. Meindl, L. Prange, H. Bock, A. Jäggi, L. Ostini (2009): GNSS processing at CODE: status report, Journal of Geodesy, 83(3-4), 353-366, doi: 10.1007/s00190-008-0281-2

Flohrer, C. (2008): Mutual Validation of Satellite-Geodetic Techniques and its Impact on GNSS Orbit Modeling. Geodätisch-geophysikalische Arbeiten in der Schweiz, 75, Schweizerische Geodätische Kommission, available at http://www.sgc.ethz.ch/sgc-volumes/sgk-75.pdf

Hackel, C., O. Montenbruck (2015): Impact of Improved Satellite Dynamics on Reduced-Dynamic Orbits, in preparation

Jäggi, A., U. Hugentobler, G. Beutler (2006): Pseudo-stochastic orbit modeling techniques for low-Earth satellites. Journal of Geodesy, 80(1), 47-60, doi: 10.1007/s00190-006-0029-9

Jäggi, A. (2007): Pseudo-Stochastic Orbit Modeling of Low Earth Satellites Using the Global Positioning System. Geodätisch-geophysikalische Arbeiten in der Schweiz, 73, Schweizerische Geodätische Kommission, available at http://www.sgc.ethz.ch/sgc-volumes/sgk-73.pdf 


\section{Literature (3)}

Jäggi, A., R. Dach, O. Montenbruck, U. Hugentobler, H. Bock, G. Beutler (2009): Phase center modeling for LEO GPS receiver antennas and its impact on precise orbit determination. Journal of Geodesy, 83(12), 11451162, doi: 10.1007/s00190-009-0333-2

Jäggi, A., H. Bock, L. Prange, U. Meyer, G. Beutler (2011a): GPS-only gravity field recovery with GOCE, CHAMP, and GRACE. Advances in Space Research, 47(6), 1020-1028, doi: 10.1016/j.asr.2010.11.008

Jäggi, A., L. Prange, U. Hugentobler (2011b): Impact of covariance information of kinematic positions on orbit reconstruction and gravity field recovery. Advances in Space Research, 47(9), 1472-1479, doi:

10.1016/j.asr.2010.12.009

Jäggi, A., H. Bock, U. Meyer (2014): GOCE precise orbit determination for the entire Mission - challenges in the final mission phase. ESA Special Publications 728 


\section{Literature (4)}

Jäggi, A., H. Bock, U. Meyer, G. Beutler, J. van den ljssel (2015a): GOCE: assessment of GPS-only gravity field determination. Journal of Geodesy, 89(1), 33-48. doi: 10.1007/s00190-014-0759-z

Jäggi, A., C. Dahle, D. Arnold, H. Bock, U. Meyer, G. Beutler, J. van den IJSSel (2015b): Swarm kinematic orbits and gravity fields from 18 months of GPS data. Advances in Space Research, in review.

Mayer-Gürr, T., K.H. Ilk, A. Eicker, M. Feuchtinger (2005): ITG-CHAMP01: a CHAMP gravity field model from short kinematic arcs over a one-year observation period. Journal of Geodesy, 78(7-8), 462-480. doi:

10.1007/s00190-004-0413-2

Meyer, U., A. Jäggi, G. Beutler, H. Bock (2015): The impact of common versus separate estimation of orbit parameters on GRACE gravity field solutions. Journal of Geodesy, 89(7), 685-696. doi: 10.1007/s00190-015-0807-3 


\section{Literature (5)}

Sośnica, K., A. Jäggi, D. Thaller, R. Dach, G. Beutler (2014a): Contribution of Starlette, Stella, and AJISAI to the SLR-derived global reference frame. Journal of Geodesy, 88(8), 789-804. doi: 10.1007/s00190-014-0722-z Sośnica, K., A. Jäggi, U. Meyer, M. Weigelt, T. van Dam, N. Zehentner, T. Mayer-Gürr (2014b): Time varying gravity from SLR and combined SLR and high-low satellite-to-satellite tracking data. GRACE Science Team Meeting 2014, 29th September to 1st October 2014, Potsdam, Germany

Sośnica, K., D. Thaller, R. Dach, P. Steigenberger, G. Beutler, D. Arnold, A. Jäggi (2015a): Satellite laser ranging to GPS and GLONASS. Journal of Geodesy, 89(7), 725-743. doi: 10.1007/s00190-015-0810-8

Sośnica, K., A. Jäggi, U. Meyer, D. Thaller, G. Beutler, D. Arnold, R. Dach (2015b): Time variable Earth's gravity field from SLR satellites. Journal of Geodesy, 89(10), 945-960. doi: 10.1007/s00190-015-0825-1 


\section{Literature (6)}

Sośnica, K. (2015): Determination of Precise Satellite Orbits and Geodetic

Parameters using Satellite Laser Ranging. Geodätisch-geophysikalische Arbeiten in der Schweiz, 93, Schweizerische Geodätische Kommission, available at http://www.sgc.ethz.ch/sgc-volumes/sgk-93.pdf

Svehla, D., M. Rothacher (2004): Kinematic Precise Orbit Determination for Gravity Field Determination, in A Window on the Future of Geodesy, edited by F. Sanso, pp. 181-188, Springer, doi: 10.1007/b139065

Visser, P., J. van den IJssel, T. van Helleputte, H. Bock, A. Jäggi, G. Beutler, D. Švehla, U. Hugentobler, M. Heinze (2009): Orbit determination for the GOCE satellite, Advances in Space Research, 43(5), 760-768, doi:

10.1016/j.asr.2008.09.016

Visser, P.N.A.M., J.A.A. van den IJssel (2015): Calibration and validation of individual GOCE accelerometers by precise orbit determination. Journal of Geodesy, in press, doi: 10.1007/s00190-015-0850-0

Zehentner, N., T. Mayer-Gürr (2015): Precise orbit determination based on raw GPS measurements. Journal of Geodesy, in review. 